\title{
Liquid Phase Supercontinuum Fiber-loop Cavity Enhanced Absorption Spectroscopy
}

\author{
Mingyun Li \\ Maanshan, Anhui, China \\ B.S. in Chemical Physics \\ University of Science and Technology of China, July 2012
}

A Dissertation presented to the Graduate Faculty of the University of Virginia in Candidacy for the Degree of Doctor of Philosophy

Department of Chemistry

University of Virginia

November 2017 


\section{Abstract}

$\mathrm{H}_{2} \mathrm{O}$ has long been an elementary compound that we would like to know its concentrations in different kinds of chemical mixtures. There are many kinds of measurements that can do this well, but in this work, we are focusing on building our own system for $\mathrm{H}_{2} \mathrm{O}$ detection by using a Cavity Enhanced Absorption Spectroscopy (CEAS) method.

A typical CEAS system is usually made up of a light source, a resonant cavity and an optical detector. The development of CEAS starts in gas phase detection, and it has been developed to liquid and solid phases in the recent decades. The detection limit is not able to match up that of gas phase sensing due to higher optical loss, but it makes a new method for condensed phase detection in low concentrations. The sensing device can be simple, and by using a fiber loop in this work, we are able to build a robust liquid phase spectrometer.

A supercontinuum is used as the light source in this project. It is a broadband light source that is generated from a simple narrowband laser beam. The laser we use is a Q-switched $1064 \mathrm{~nm}$ infrared laser, and the supercontinuum we get can cover the region between 600 and $1600 \mathrm{~nm}$. A supercontinuum can have the same characteristics as the pump laser such as spatial coherence and high power, and it can cover a wider spectral range at the same time. 
Several liquid phase sensors are built to perform sensing of $\mathrm{H}_{2} \mathrm{O}$ in different liquids, and each sensor is tested for its stability and detection limit. Fiber tapers, side-polished fibers and a mirror reflection device are tested in this project. Every sensor has some unique properties and its own advantages and limitations.

The main liquid samples we test begin with solutions of $\mathrm{H}_{2} \mathrm{O}$ in $\mathrm{D}_{2} \mathrm{O}$. These inorganic samples allow us understand the detection ability of our system and its signal to noise level. After that some organic samples are tested, and here $\mathrm{H}_{2} \mathrm{O}$ concentration is the main interest as well. Several organics with low $\mathrm{H}_{2} \mathrm{O}$ solubility are tested and results show a slightly higher noise level for them but some absorbance can also be found in the spectra. 


\section{Table of Contents}

Abstract

Chapter 1: Introduction of Cavity Enhanced Absorption Spectroscopy (CEAS), Cavity

Ring-down Spectroscopy (CRDS) and Supercontinuum.............................................. 1

1.1 Cavity Ring-down Spectroscopy (CRDS) .................................................. 1

1.2 Cavity Enhanced Absorption Spectroscopy (CEAS) ........................................ 5

1.3 Expansion of CRDS and CEAS Techniques to Condensed Phase .......................... 7

1.4 Supercontinuum and Photonic Crystal Fiber (PCF) ........................................ 9

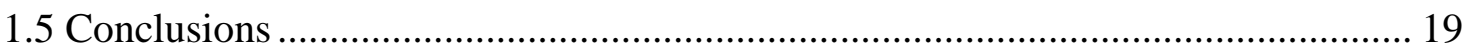

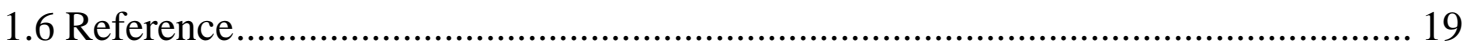

Chapter 2: Fiber Based Cavity Enhanced Absorption Spectroscopy............................... 25

2.1: Fiber-loop Resonator and Supercontinuum in the Resonator .............................. 25

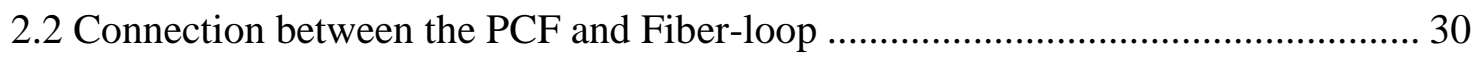

2.3 Theoretical and Experimental Loss of the Fiber-loop Resonator .......................... 36

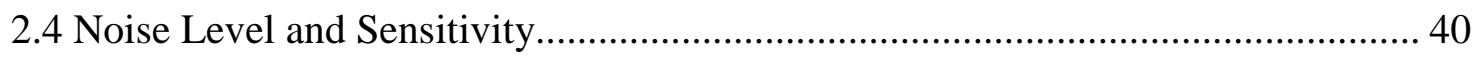

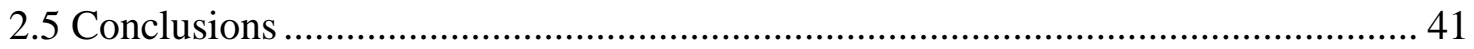

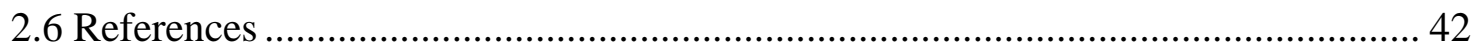

Chapter 3 Liquid Phase Sensing Theories and Methods ............................................ 45

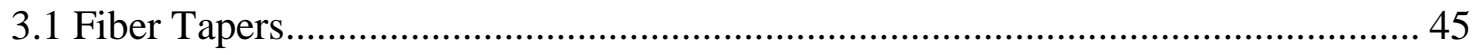

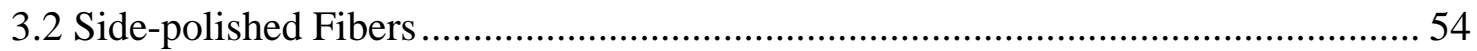

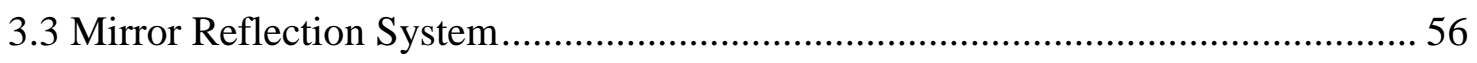

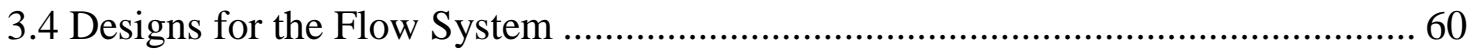

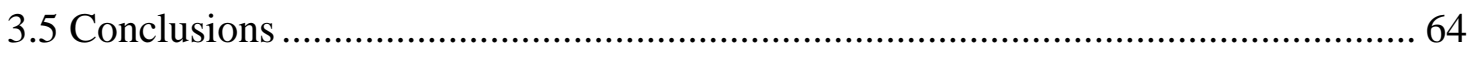

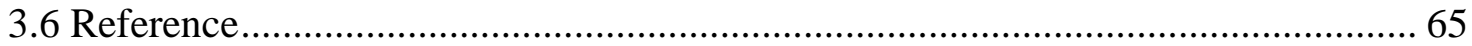

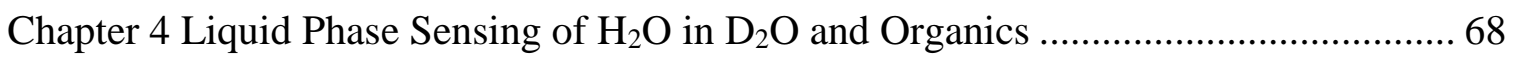

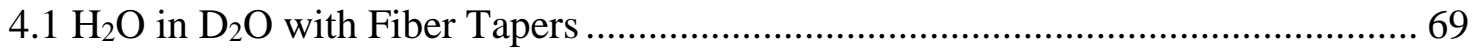

$4.2 \mathrm{H}_{2} \mathrm{O}$ solutions in $\mathrm{D}_{2} \mathrm{O}$ with Side-polished Fiber Sensors ................................. 72

4.3. Results for Mirror Reflection Device .............................................................. 78

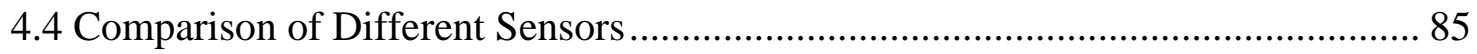




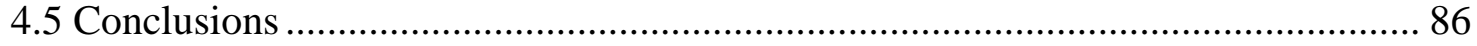

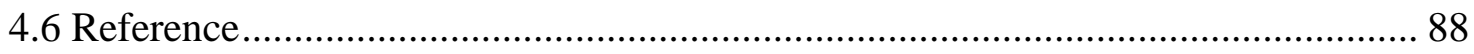

Chapter 5 Applications and Future Work ……………….............................................. 91

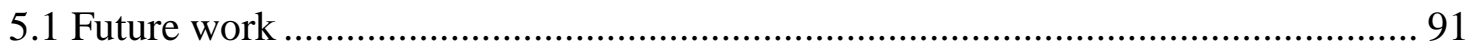

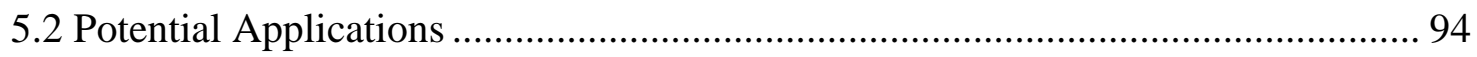

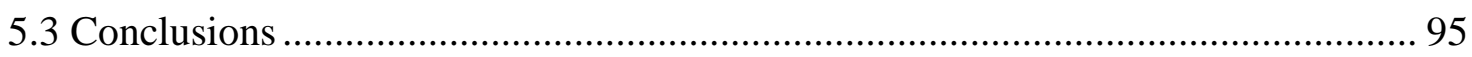

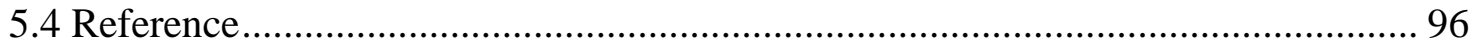

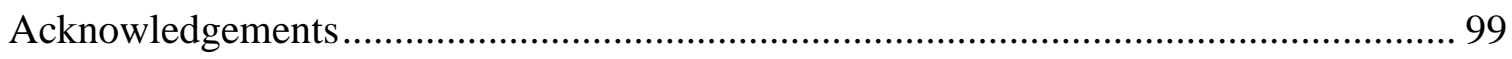




\section{Chapter 1: Introduction of Cavity Enhanced Absorption Spectroscopy (CEAS), Cavity Ring-down Spectroscopy (CRDS) and Supercontinuum}

Cavity Ring-down Spectroscopy (CRDS) [1] and Cavity Enhanced Absorption Spectroscopy (CEAS) [2] are well developed in the recent several decades as methods of high sensitivity spectroscopy measurements. They were first developed in gas phase sensing [3-5], and further expanded to liquid and solid phases in recent decade. [6, 7] CEAS and CRDS methods are famous for their simple setup, fast sensing and high sensitivity. In gas phase sensing, these techniques can easily reach a part per billion or even part per trillion level [5]. It is usually worse for liquid phase sensing due to lower reflectivity and thus shorter sample sensing optical path length and larger scattering losses, but a relatively high sensitivity can still be achieved [6]. In this project, the work is done on building a liquid phase CEAS sensing device in the near infrared region.

\subsection{Cavity Ring-down Spectroscopy (CRDS)}

The Cavity Ring-down spectroscopy (CRDS) was first developed for trace gas detection by O'Keefe and Deacon in the late 1980s. [1] After rapidly developing for several decades, this technique has grown to a very mature method in gas phase detection and is no longer limited to research labs; development of portable devices has made it possible for it to work anywhere. [5] A simple CRDS spectrometer schematics is shown in Figure 1.1. The basic 
idea of the CRDS method is to use two high reflective mirrors (usually $>99.9 \%$ reflectivity) to create an optical cavity. When the mirrors form a stable optical cavity, a high intensity pulsed light is introduced into the cavity, it will reflect between the two or more mirrors to increase the optical path. When the input light is over, an exponential decay can be observed on the output light intensity to create a so-called "ring-down". A time constant of the decay is defined as the "ring-down time" and is usually represented by $\tau$. It is the time it takes to reach $1 / \mathrm{e}$ of the maximum output intensity. The ring-down time is inversely proportional to the loss of the optical cavity, so if an absorbing gas is introduced into the cavity, the absorbance would make the cavity loss increase so the ring-down time is reduced. The ring-down time change will allow us to have a precise quantitative measurement of the optical extinction for a sample inside the cavity.
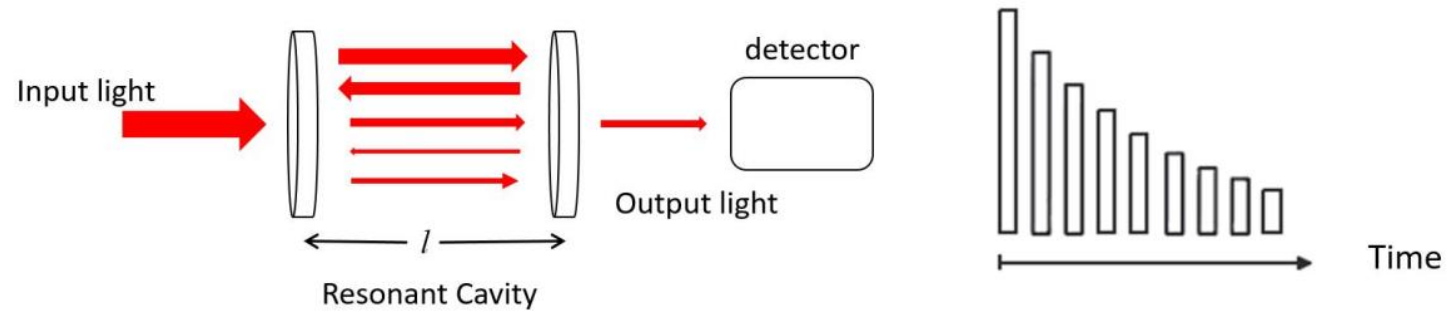

Figure 1.1: Schematic of a ring-down cavity and ring-down signal

Although CRDS theories were first built on pulsed laser input [1] and the laser beam has to be cut for the intensity inside cavity to decay and form a "ring-down", continuous-wave (CW) lasers do help on CRDS sensing in some other ways. CW lasers are easier to find and operate, less expensive and usually safer than a pulsed laser with similar output power. 
More importantly, using a CW laser can help to more selectively excite the ground mode $\left(\mathrm{TEM}_{00}\right)$ of the cavity, thus to minimize the influence from higher order cavity modes on the ring-down signal. A more stable signal gives more stable ring-down time so that the measurement is more sensitive because of less fluctuation on the ring-down time (Usually ring-down times for high order cavity modes are lower than that for the ground mode). Nowadays, almost all CRDS works are using CW lasers as their light sources. [8-10]

Because of its high sensitivity and robust and simple setup, the CRDS technique has been developed and widely used in more and more regions ever since it has been demonstrated. Examples like environmental detection [11], human health monitoring [12] and fast or simultaneous measurements [3] show that it has a very wide application range. There are commercial CRDS spectrometers on sale from several manufactures like Tiger Optics [13] and Picarro [14]. Now with the development of CRDS in smaller setups [3] and even to condensed phases [6] other than gaseous detection, this technique may have much broader application prospects.

To show how to get a ring-down decay, we will consider an optical cavity with total length $L$ and two mirrors with reflectivity $R$, and ignoring higher order cavity modes to start with [15]:

$$
I(t) \propto \int_{0}^{\infty} I_{0}(v) \exp \left[-\frac{t}{\tau(v)}\right] d v
$$

Where $I_{0}(v)$ is light intensity when the pulse laser ends and $\tau$ is the ring-down time. 
The ring-down time is related to the loss inside a resonant cavity:

$$
\tau(v)=\frac{d}{c\left\{|\ln [R(v)]|+\sum_{i} \sigma_{i}(v) \int_{0}^{l} N_{i}(x) d x\right\}}
$$

Where $c$ is the speed of light in the cavity and $d$ is the separation of the two mirrors, here will be equal to $l$. The reflectivity of the mirrors is usually higher than 0.99 , so it is reasonable to estimate that $|\ln R|=1-R . \sigma_{i}(v)$ is the frequency-related absorption cross section and $\int_{0}^{l} N_{i}(x) d x$ stands for a number density of the absorption species. Since the frequency is usually narrow and number density is homogeneous inside cavity, the ringdown time can be written as:

$$
\tau(v)=\frac{d}{c[(1-R)+\alpha(v) d]}
$$

Where $\alpha$ is the molecular absorption coefficient of the absorbing species (usually with a unit of $\left.\mathrm{cm}^{-1}\right)$.

Thus for a ring-down decay at a certain frequency, it can be written as:

$$
I(t)=I(0) \exp \left[-\frac{t}{\tau}\right]
$$

And if we compare ring-down times with and without sample species, the absorption coefficient of a sample can be written as:

$$
\alpha(v)=\frac{1}{c \tau(v)}-\frac{1}{c \tau_{0}(v)}
$$

Where $\tau_{0}$ is ring-down time for empty cavity and $\tau$ is ring-down time with sample.

Furthermore we are able to get the absorption cross section to be:

$$
\sigma_{i}(v)=\frac{\alpha(v)}{N_{i}}
$$


It is easy to see from (1.5) that the absorbing feature of a specific species is only dependent on the ring-down time, which helps to minimize the influence from intensity fluctuation and optical loss outside the cavity resonator. The elimination of most noise in a spectroscopy measurement makes the final result more accurate in CRDS.

\subsection{Cavity Enhanced Absorption Spectroscopy (CEAS)}

The Cavity Enhanced Absorption Spectroscopy (CEAS) has almost the same working theories as a CRDS. [16] It starts with an optical cavity and two high reflective mirrors as well, so we are able to enhance the detection limit by making a high optical path length. The main difference between a CRDS and a CEAS is that in a CEAS detection, we usually measure an output spectrum directly by intensity in a wavelength range while in a CRDS the measured intensity is converted to a ring-down time. Instead of calculating a ring-down time for a single wavelength, we can get an absorption character for one or several samples in a wider spectral range we want to know at the same time. By Beer's law, an absorption coefficient can be calculated at every wavelength point we are interested in.

The Beer's law is the main equation we use in a CEAS detection:

$$
I=I(0) \exp \left[-\sum_{i} \sigma_{i} \int_{0}^{l} N_{i}(x) d x\right]
$$

Or:

$$
A=-\log \left[\frac{I}{I(0)}\right]=\sum_{i} \sigma_{i} \int_{0}^{l} N_{i}(x) d x
$$

With a homogeneous sample species we can get the simplest Beer's law: 


$$
A=\sum_{i} \sigma_{i} N_{i} L=-\log \left[\frac{I}{I(0)}\right]
$$

Where $\sigma_{i}$ is the molecular absorption cross section, $N_{i}$ is the number density and $L$ is path length of light in the samples, and $A$ is the total absorbance.

Compared to a CRDS, the advantage of a CEAS measurement is obvious, however, the limitation of it is also easy to find. In a CRDS method, the final calculation gives us a ringdown time as the character to show the sample properties, but in a CEAS test, the main character changes to optical intensity itself. It is a more straightforward character but the intensity is usually not a very stable output. Power fluctuation, loss variation in the setup or other intensity random shifts can make the final output intensity fluctuates from time to time. The CRDS can get rid of this problem by detecting ring-down time instead of direct intensity but CEAS does not have such a character so intensity fluctuation is a point we need to think of in this project.

Based on its unique characteristics in spectroscopic measurements, there are many areas where CEAS methods can be useful. In some recent works, some incoherent light sources are used $[17,18]$, while in others there are requirements of multiple species or multiple wavelength detections simultaneously $[19,20]$. When expanded to liquid or solid phase detections, CEAS has its unique advantages in more aspects such as detection of refractive index [21] and microfluidic chip measurements [22] which demands only very little sample volume. In this work, we are using CEAS method to do liquid phase detections because a 
broadband light source is put in use. Here we will briefly demonstrate how CEAS can be adapted in liquid phase sensing. A whole description of how our system is designed and set will be fully introduced in Chapter 2 .

\subsection{Expansion of CRDS and CEAS Techniques to Condensed Phase}

The applications of CRDS and CEAS methods in gas phase have been well developed in the recent decades, and it has expanded to liquid and solid phases in the most recent decades [23]. Some unique optical cavities are designed to make them work in different circumstances. The ideas of using Brewster angle or total reflection have significantly expand the region where these techniques can make into use.

For condensed phases, the cavity design is separated to two main methods. The first one is to build a cavity similar as in the gas phase. A typical design is to build a gap on a singlemode fiber, and to fill the region between with the liquid species in a cuvette [24], or a waveguide [25] put between the fiber ends to work as an optical cavity. Such designs are very easy to realize but sometimes they can be unstable since the samples and fiber components are exposed outside and easy to be influenced by surrounding environments. High optical loss is another issue. Components like a cuvette sometimes have low reflectivity on their walls which can further lower the total reflectivity. The finesse for a liquid phase cavity is usually not as high as one built for gas phase. In this case for condensed phase CRDS and CEAS, the optical path length or ring-down time is always 
much smaller than gas phase devices.

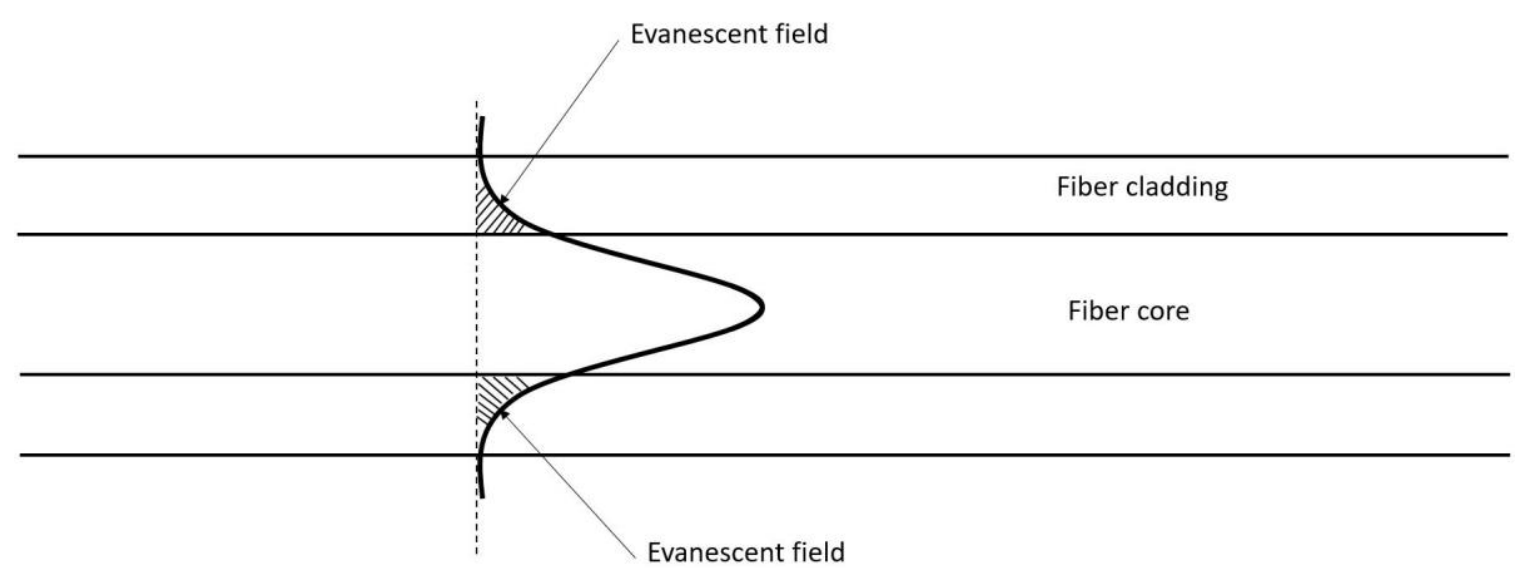

Figure 1.2: Schematic of evanescent field sensing. When a light is travelling in a fiber core in total internal reflection, there will electromagnetic field in a region around the fiber core. It is called the evanescent field and it usually spread from nanometers to micros away from the core.

The second design is to remain in an intact optical fiber that no gaps or etches are formed on it. In this kind of designs evanescent wave spectroscopy (EWS) [26-28] is usually the simplest way to make the whole system work. As show in Figure 1.2, when a light is travelling inside an optical fiber in total reflection, a portion of the light can still be exposed outside the core of the fiber. The percentage of the exposed light is related to the distance to the core and the total reflection angle. Theoretically, if the cladding of a fiber can be thin enough to less than 1 micron, a considerable amount of the light can be exposed in a region within a few microns outside the fiber. The evanescent field area (so called penetration depth into the cladding or surround region) also varies for different fiber components. We will further demonstrate this in details in Chapter 3. 
Based on the EWS, the smaller incident angle and the closer to the fiber core, the more evanescent intensity will be detected [29]. Thus, several liquid phase CRDS and CEAS sensing methods are developed aiming at making claddings thinner on an optical fiber. Side-polished fibers [30] and fiber tapers [29] are two typical examples of them. They both have their own strong points but some limitations as well. A side-polished fiber is made by removing cladding of a fiber from one side to near its core, while a taper is made by heating and pulling a fiber to make a tapered region so both the core and cladding will be thinner. In this work, we tried to perform some experiments on both sensors and to compare their sensing abilities. The structure and some measurement results on side-polished fibers and taper sensors will be further described in chapter 3 .

\subsection{Supercontinuum and Photonic Crystal Fiber (PCF)}

In order to build a simple but stable CEAS apparatus, a stable and high intensity light source is in need. In this project what we choose is a supercontinuum [31, 32]. A supercontinuum is a broadband light source that is generated from a narrowband laser source. It is called a supercontinuum because it has a very broad wavelength coverage. Our self-generated supercontinuum can cover a region from $600 \mathrm{~nm}$ to $1600 \mathrm{~nm}$. In some other works, a supercontinuum is able to cross a much broader region [33]. A supercontinuum is suitable for this liquid CEAS sensing not only because it is easy to generate and its stability, but also it has the advantages that comes from the original pumping laser source such as great spatial coherence and high intensity. 
The description of the nonlinear optical effects in a supercontinuum generation process usually starts with the Maxwell Equations [34]. We will briefly demonstrated some basics of it mainly related to nonlinear effects in fiber optics. We know that from Maxwell Equations the relationship between induced polarization $\mathrm{P}$ and strength of electric field $\mathrm{E}$ is:

$$
\boldsymbol{P}(t)=\chi \boldsymbol{E}(t)
$$

Where $\chi$ is the linear susceptibility, which means in linear response, polarization is proportional to the electric field. If the electric field became larger and larger to near the atomic electric field strength, however, the response will not be harmonic any more. In such situation of high field response, the equation above will become:

$$
\boldsymbol{P}(t)=\sum_{n} \chi^{(n)} \boldsymbol{E}(t)^{\boldsymbol{n}}
$$

where $\chi^{(1)}$ is the normal linear susceptibility and $\chi^{(n)}(n>1)$ are the nth- order nonlinear susceptibilities. $\chi^{(n)}$ is also responsible for the nonlinearity of the nth-harmonic generation.

In optical fibers, the even order susceptibilities are all 0 , so the majority of the nonlinearity is coming from the third susceptibility. The main phenomenon of this nonlinear effect is the refractive index on fiber material. Commonly the nonlinear refractive index can be written as:

$$
n(v, I)=n(v)+n_{2} I
$$

Where $I$ is the light intensity inside a fiber. This equation shows that the refractive index 
of an optical fiber changes linearly with the light intensity applied inside. This is the socalled self phase modulation process [36]. It is a phase shift on the optical field propagating inside an optical fiber inducted by itself, and it makes a great contribution to the nonlinear optics in a photonic crystal fiber we will discuss below.

There are several ways demonstrated before as a generation method for a supercontinuum $[32,35,37]$, and the main difference among all methods are the transition media used. Media in both liquid and solid phases have been demonstrated as great generation methods for a supercontinuum. While the media may be different, the theories are always to introduce nonlinear optics to the light beam that is travelling inside a specific transition medium. A Photonic Crystal Fiber (PCF) [37] is used as a generation medium in this work. The structure and the nonlinear optical effects in an optical fiber will be demonstrated here.

A Photonic Crystal Fiber (PCF) is a special kind of optical fiber that uses index waveguiding to achieve nonlinear optical effects. It is first introduced and made in early 1990s' $[38,39]$, and it is called photonic crystal because the original theory was to use photonic bandgap to achieve the construction of this kind of fibers. A core dopant was used in the early works to make a PCF, and nowadays the dopants are replaced by simply hollow transverse air holes in the microstructure of the core. This makes the main difference between a PCF and a conventional single-mode fiber, one has holes around the core and the other does not. So the most commonly PCF now is made from pure silica as well, and 
it is the air holes that do the work of nonlinear optics and confinement.

The size of a PCF is similar as a standard single mode fiber. The one we use in this project has a core diameter of $5 \mu \mathrm{m}$, and a cladding diameter of $125 \mu \mathrm{m}$. As show in Figure 1.3, the cross section of a PCF clearly demonstrates the core and cladding of a PCF and the hollow air holes around the core. Expect for the core and cladding diameters, there are a few other crucial parameters that determines the nonlinear optical effect for one specific $\mathrm{PCF}$, the air hole diameter $d$, the distance between the holes $\Lambda$, and the formation shape and size of all the holes, such as a square, a hexagon or a rectangle. The PCF used here has all the air holes forms a shape of hexagon as shown in Figure 1.3, but the size and number of the holes are just schematic to show how they are arranged in the fiber. 


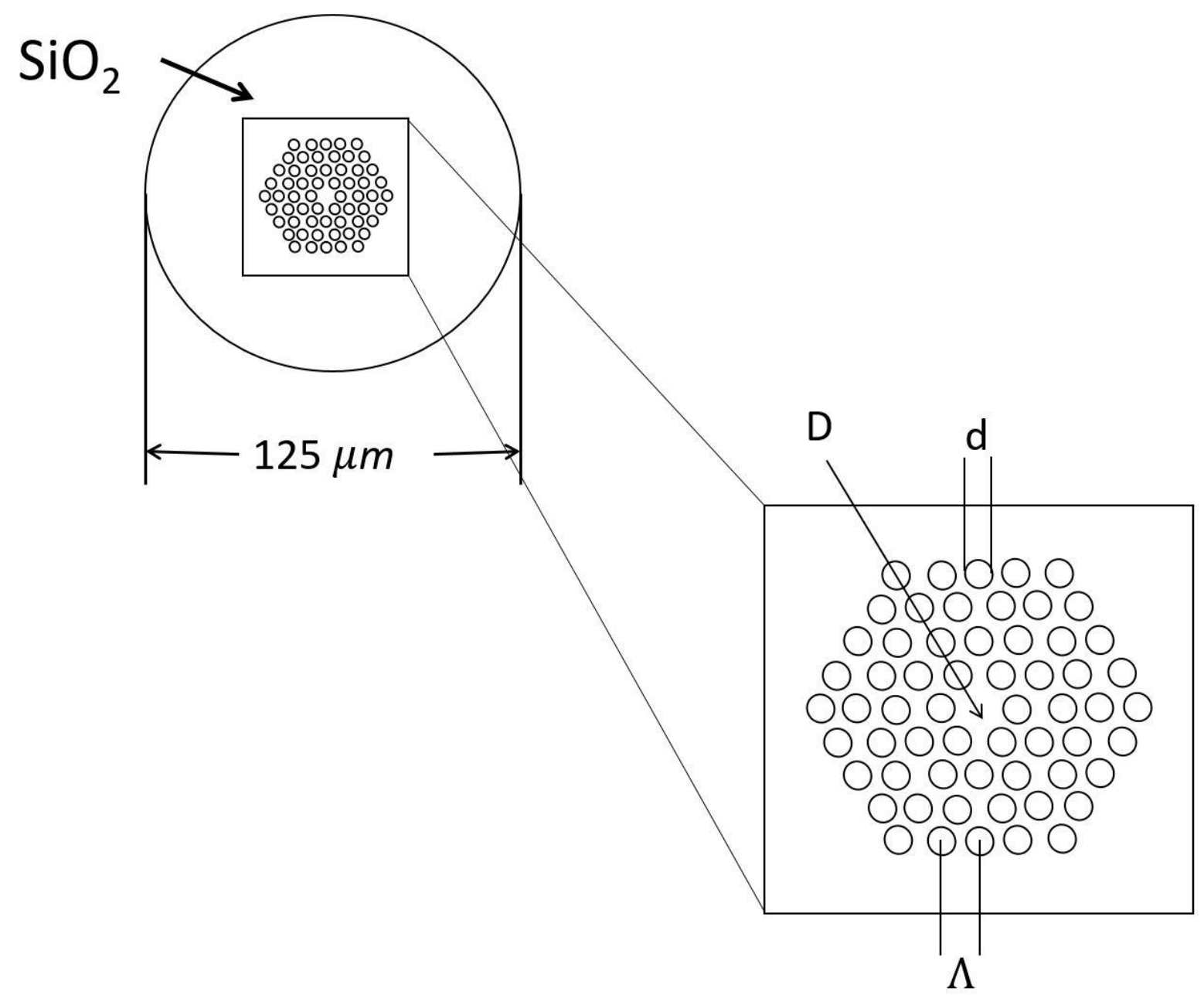

Figure 1.3: Schematic of the cross section of a Photonic Crystal Fiber (PCF) in this work, the air holes form a shape of hexagon, several important parameters that define the nonlinear optics for a PCF, $D$ : fiber core diameter; $d$ : hollow air hole diameter; and $\Lambda$ : distance between air holes next to another.

The construction of a PCF is also similar as a single-mode fiber. As listed in ref. [38], the construction of the air holes include using a drawing tower the same as conventional fibers, a fiber preform and capillary tubes. Those capillary tubes are arranged in the fiber preform as in the microstructure of the wanted PCF. The raw material is then heated in the preform and drawn out on a fiber spool using the drawing tower. This process is simple and not 
difficult to realize, furthermore, it also helps to keep the newly manufactured PCF stable and air holes do not collapse in or after the process. A PCF with air holes as small as $1.5 \mu \mathrm{m}$ has been demonstrated and manufactured in ref. [39] with a diameter accuracy of less than $1 \%$ fluctuation.

The nonlinear effect in the generation of a supercontinuum could be complicated, and it is not only depend on the transverse media but also on the wavelength and power of the pump laser. Even with the same medium such as PCFs, the difference in microstructures of different fibers will have different dispersion properties. For each designed PCF, the pump source must be near its zero dispersion wavelength to generate a supercontinuum. The PCF we are using has a zero dispersion wavelength of $1040 \mathrm{~nm}$ and the pump laser source is at $1064 \mathrm{~nm}$. The main nonlinear optics for a supercontinuum generation in a PCF is called four-wave mixing and self phase modulation [40, 41]. We will demonstrate a detailed discussion of the nonlinear effects on PCFs.

Degenerate four-wave mixing is the annihilation of two pumped protons that are converted into two sidebands that are frequency downshifted (Stokes) or upshifted (anti-Stokes). [41] Self phase modulation gives the phase match to realize degenerate four-wave mixing. The phase match and conservation of energy equation can be given as follows:

$$
k_{\text {pump }}=k_{\text {signal }}+k_{\text {idler }}+2 \gamma P
$$




$$
2 \omega_{\text {pump }}=\omega_{\text {signal }}+\omega_{\text {idler }}
$$

where $k$ are wavevectors of fiber modes and $\omega$ are the frequencies of pump, signal (antiStokes) and idler (Stokes). $\gamma$ is the nonlinear coefficient of photonic crystal fiber and $P$ is the peak power of the pump. $\gamma$ can be written as:

$$
\gamma=\frac{2 \pi n_{2}}{\lambda A_{e f f}}
$$

where $\lambda$ is the pump wavelength and $A_{e f f}$ is effective area of the fiber. $n_{2}$ is the nonlinear refractive index of fused silica, $n_{2}=2 \times 10^{-20} \mathrm{~m}^{2} / \mathrm{W}$. [41]

Maximum gain in sidebands requires zero phase mismatch. The phase mismatch of the photonic crystal fiber is:

$$
\kappa=\Delta k_{m}+\Delta k_{w}+\Delta k_{N L}=0
$$

where $\Delta k_{m}$ is the phase mismatch from material dispersion, $\Delta k_{w}$ is the phase mismatch from waveguide dispersion, and $\Delta k_{N L}$ is the phase mismatch from nonlinear effects. The three parameters can be written as:

$$
\begin{gathered}
\Delta k_{m}=\frac{n_{3} \omega_{3}+n_{4} \omega_{4}-2 n_{1} \omega_{1}}{c} \\
\Delta k_{w}=\frac{\Delta n_{3} \omega_{3}+\Delta n_{4} \omega_{4}-2 \Delta n_{1} \omega_{1}}{c} \\
\Delta k_{N L}=2 \gamma P
\end{gathered}
$$

where $n$ are the material indexes of refraction, $\Delta n$ are the change in the material indexes of refraction by waveguide dispersion. Because the pump frequency lies in the anomalous dispersion region of the fiber, $\Delta k_{m}$ is negative. $\Delta k_{N L}$ is then used to compensate for the contributions of $\Delta k_{m}$ and $\Delta k_{w}$ to give $\kappa=0$. 
With the developments in the recent few decades, the structure and stability of PCFs become much better, in the meantime, by using a PCF instead of other generation media, the requirement of the pump laser in supercontinuum generation becomes much lower than before as well. When a supercontinuum is first generated [35], the coverage is very small (only $400 \mathrm{~nm}$ to $700 \mathrm{~nm}$ ), and the conditions usually require pump laser power as high as up to milliwatts per pulse, and a pulse as short as picosecond lasers [31, 35]. With the introduction of PCFs, however, these requirements have been significantly decreased to be much less expensive and more importantly, far less critical conditions.

Table 1.1: Parameters of the Photonic Crystal Fiber [42]

\begin{tabular}{|l|l|l|l|}
\hline \multicolumn{2}{|l|}{ Physical Properties } & \multicolumn{2}{l}{ Optical Properties } \\
\hline Material & Pure silica & Zero-dispersion wavelength & $1040 \pm 10 \mu m$ \\
\hline Cladding diameter & $125 \pm 3 \mu m$ & Cut-off wavelength & $<1000 \mathrm{~nm}$ \\
\hline \multirow{2}{*}{ Coating diameter } & $244 \pm 10 \mu m$ & Nonlinear coefficient @ 1060nm & $11(\mathrm{~W} \cdot \mathrm{km})^{-1}$ \\
\hline \multirow{2}{*}{ Coating material } & \multirow{2}{*}{ Acrylate } & Attenuation @ 1040nm & $<3 \mathrm{~dB} / \mathrm{km}$ \\
\hline \multirow{2}{*}{ Core diameter } & $4.8 \pm 0.2 \mu m$ & Attenuation @ 1550nm & $<2.5 \mathrm{~dB} / \mathrm{km}$ \\
\hline \multirow{2}{*}{} & & Attenuation @ 600nm & $<25 \mathrm{~dB} / \mathrm{km}$ \\
\cline { 2 - 4 } & Mode field diameter & $4.0 \pm 0.2 \mu m$ \\
\cline { 2 - 4 } & & Numerical aperture @ 1060nm & $0.20 \pm 0.05$ \\
\cline { 2 - 4 } & & $\Delta n @ 1060$ nm & $1.7 \times 10^{-4}$ \\
\cline { 2 - 4 } & & Polarization extinction ratio & $>20 \mathrm{~dB}$ \\
\cline { 2 - 4 } & &
\end{tabular}


In this project, $\sim 15 \mathrm{~m}$ of PCF (NKT Photonics, SC-5.0-1040) is used in generation of a supercontinuum and Table 1.1 shows several parameters of this PCF. The pump power is a Q-switched Nd:YAG laser centered at $1064 \mathrm{~nm}$. The repetition rate on the pump laser is set at a few tens of $\mathrm{kHz}$ and a maximum time averaged power output on this laser is only 1.2W. The pulsed laser is free-space coupled into the PCF and a microscope is used to focus the laser beam on the input end of the PCF. Normally a transmission rate of $\sim 50 \%$ of the laser can be reached and the supercontinuum can cover the region from $600 \mathrm{~nm}$ to $1600 \mathrm{~nm}$. Figure 1.4 gives an example of the spectrum of this supercontinuum looks like. Only $900 \mathrm{~nm}$ to $1700 \mathrm{~nm}$ is recorded because the coverage of our spectrometer (Ocean Optics, NIRQuest 512) is in this region. The pulse mode laser is set at $20 \mathrm{kHz}$ repetition rate and spectra at different pump powers are recorded to show how the supercontinuum expanded with the increase of pump power. All spectra are taken with $20 \mathrm{~ms}$ integration time and 100 times average, and the intensity is normalized against a standard Halogen light source to give a relative power.

Shortly after it was first introduced in the 1970s [31, 35], supercontinuum light sources show their potential uses in many ways for its obvious advantages over other light sources. Now with the developing of PCF techniques, it has become much easier to realize the generation process. [32] Its higher intensity, broader spectral range, lower cost and higher reliability than most other light sources has made a supercontinuum the first choice when people think of using broadband light sources. $[19,43]$ Same as CRDS spectrometers, there 
are more companies now making manufactured supercontinuum light sources [44, 45], mostly based on nonlinear fiber optics. The rapidly growing market requirements stimulates the development of supercontinuum technology as well. While more people

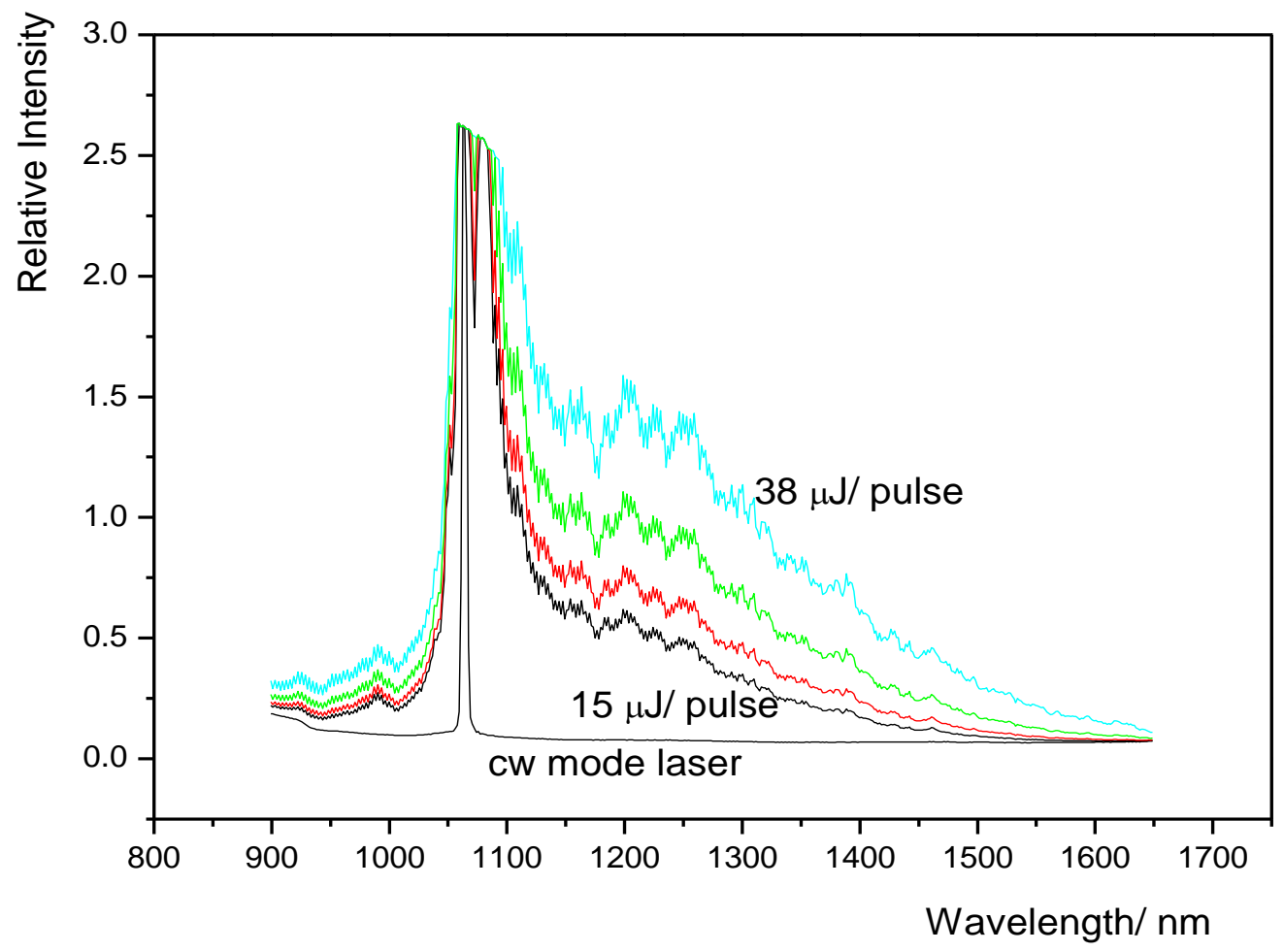

Figure 1.4: Figure of the supercontinuum output we have in this work, different colors stand for different power of the pump laser to show how the supercontinuum grow with pump power. Spectrum in the bottom is the pump laser at $1064 \mathrm{~nm}$, which further confirms the super-broadening effect in the generation of supercontinuum.

purchase a supercontinuum for very specific applications, there are only several kinds on sale right now. Being still in very fast developments, we believe it will have a wider region for applications in the future. 


\subsection{Conclusions}

In this project, a fiber based liquid phase CEAS device will be demonstrated. A supercontinuum is used as the light source and a fiber-loop is built as a liquid phase optical resonator. The work is done in the near infrared region based on the coverage of the supercontinuum source and the spectrometer. The design of the whole device will be introduced first in the following chapter.

\subsection{Reference}

1. O'Keefe, Anthony, and David AG Deacon. "Cavity ring-down optical spectrometer for absorption measurements using pulsed laser sources." Review of Scientific Instruments 59, no. 12 (1988): 2544-2551.

2. Engeln, Richard, Giel Berden, Rudy Peeters, and Gerard Meijer. "Cavity enhanced absorption and cavity enhanced magnetic rotation spectroscopy." Review of scientific instruments 69, no. 11 (1998): 3763-3769.

3. Jiang, Chenyu, Meixiu Sun, Zhennan Wang, Zhuying Chen, Xiaomeng Zhao, Yuan Yuan, Yingxin Li, and Chuji Wang. "A portable real-time ringdown breath acetone analyzer: toward potential diabetic screening and management." Sensors 16, no. 8 (2016): 1199.

4. Paldus, B. A., C. C. Harb, T. G. Spence, R. N. Zare, C. Gmachl, F. Capasso, D. L. Sivco, J. N. Baillargeon, A. L. Hutchinson, and A. Y. Cho. "Cavity ringdown spectroscopy 
using mid-infrared quantum-cascade lasers." Optics letters 25, no. 9 (2000): 666-668.

5. Kassi, Samir, and Alain Campargue. "Cavity ring down spectroscopy with $5 \times 10-13$

cm- 1 sensitivity." The Journal of chemical physics 137, no. 23 (2012): 234201.

6. Xu, Shucheng, Guohe Sha, and Jinchun Xie. "Cavity ring-down spectroscopy in the liquid phase." Review of scientific instruments73, no. 2 (2002): 255-258.

7. Waechter, Helen, Jessica Litman, Adrienne H. Cheung, Jack A. Barnes, and Hans-Peter Loock. "Chemical sensing using fiber cavity ring-down spectroscopy." Sensors 10, no. 3 (2010): 1716-1742.

8. Tan, Y., J. Wang, C-F. Cheng, X-Q. Zhao, A-W. Liu, and S-M. Hu. "Cavity ring-down spectroscopy of the electric quadrupole transitions of $\mathrm{H} 2$ in the $784-852 \mathrm{~nm}$ region." Journal of Molecular Spectroscopy 300 (2014): 60-64.

9. Romanini, D., A. A. Kachanov, N. Sadeghi, and F. Stoeckel. "CW cavity ring down spectroscopy." Chemical Physics Letters 264, no. 3-4 (1997): 316-322.

10. Dudek, John B., Peter B. Tarsa, Armando Velasquez, Mark Wladyslawski, Paul Rabinowitz, and Kevin K. Lehmann. "Trace moisture detection using continuous-wave cavity ring-down spectroscopy." Analytical chemistry 75, no. 17 (2003): 4599-4605.

11. Atkinson, Dean B. "Solving chemical problems of environmental importance using cavity ring-down spectroscopy." Analyst 128, no. 2 (2003): 117-125.

12. Thorpe, Michael J., David Balslev-Clausen, Matthew S. Kirchner, and Jun Ye. "Cavityenhanced optical frequency comb spectroscopy: application to human breath analysis." Optics Express 16, no. 4 (2008): 2387-2397. 
13. Available at: http://www.laserfocusworld.com/articles/2012/10/tiger-optics-laserbased-analyzer.html

14. Available at: https://www.picarro.com/technology/cavity_ring_down_spectroscopy

15. Berden, Giel, Rudy Peeters, and Gerard Meijer. "Cavity ring-down spectroscopy: Experimental schemes and applications." International Reviews in Physical Chemistry 19, no. 4 (2000): 565-607.

16. Paldus, Barbara A., and Alexander A. Kachanov. "An historical overview of cavityenhanced methods." Canadian Journal of Physics 83, no. 10 (2005): 975-999.

17. Fiedler, Sven E., Achim Hese, and Albert A. Ruth. "Incoherent broad-band cavityenhanced absorption spectroscopy." Chemical physics letters 371, no. 3 (2003): 284294.

18. Ruth, Albert A., Sophie Dixneuf, and Johannes Orphal. "Laser-induced plasmas in ambient air for incoherent broadband cavity-enhanced absorption spectroscopy." Optics express 23, no. 5 (2015): 6092-6101.

19. Langridge, J. M., T. Laurila, R. S. Watt, R. L. Jones, C. F. Kaminski, and J. Hult. "Cavity enhanced absorption spectroscopy of multiple trace gas species using a supercontinuum radiation source." Optics Express 16, no. 14 (2008): 10178-10188.

20. Romanini, D., M. Chenevier, S. Kassi, M. Schmidt, C. Valant, M. Ramonet, J. Lopez, and H-J. Jost. "Optical-feedback cavity-enhanced absorption: a compact spectrometer for real-time measurement of atmospheric methane." Applied Physics B 83, no. 4 (2006): 659-667. 
21. Flores, J. Michel, R. A. Washenfelder, Gabriela Adler, H. J. Lee, Lior Segev, Julia Laskin, Alexander Laskin, S. A. Nizkorodov, S. S. Brown, and Yinon Rudich. "Complex refractive indices in the near-ultraviolet spectral region of biogenic secondary organic aerosol aged with ammonia." Physical Chemistry Chemical Physics 16, no. 22 (2014): 10629-10642.

22. Nitkowski, Arthur, and Michal Lipson. "Cavity-enhanced on-chip absorption spectroscopy." U.S. Patent 8,992,836, issued March 31, 2015.

23. Thompson, Jonathan E. "Cavity-Enhanced Spectroscopy in Condensed Phases: Recent Literature and Remaining Challenges." Journal of Spectroscopy 2017 (2017).

24. Islam, Meez, L. Nitin Seetohul, and Zulfiqur Ali. "Liquid-phase broadband cavityenhanced absorption spectroscopy measurements in a $2 \mathrm{~mm}$ cuvette." Applied spectroscopy 61, no. 6 (2007): 649-658.

25. Bescherer, Klaus, Jack A. Barnes, and Hans-Peter Loock. "Absorption measurements in liquid core waveguides using cavity ring-down spectroscopy." Analytical chemistry 85, no. 9 (2013): 4328-4334.

26. Tarsa, Peter B., Paul Rabinowitz, and Kevin K. Lehmann. "Evanescent field absorption in a passive optical fiber resonator using continuous-wave cavity ring-down spectroscopy." Chemical Physics Letters 383, no. 3 (2004): 297-303.

27. Avino, S., C. Richmond, A. Giorgini, P. Malara, R. Zullo, P. De Natale, and G. Gagliardi. "High-sensitivity ring-down evanescent-wave sensing in fiber resonators." Optics letters 39, no. 19 (2014): 5725-5728. 
28. Waechter, Helen, Jessica Litman, Adrienne H. Cheung, Jack A. Barnes, and Hans-Peter Loock. "Chemical sensing using fiber cavity ring-down spectroscopy." Sensors 10, no. 3 (2010): 1716-1742.

29. Ahmad, Mohammad, and Larry L. Hench. "Effect of taper geometries and launch angle on evanescent wave penetration depth in optical fibers." Biosensors and Bioelectronics 20, no. 7 (2005): 1312-1319.

30. Tseng, Shiao-Min, and Chin-Lin Chen. "Side-polished fibers." Applied optics 31, no. 18 (1992): 3438-3447.

31. Alfano, Robert R. "The supercontinuum laser source." (1989): 458.

32. Dudley, John M., Goëry Genty, and Stéphane Coen. "Supercontinuum generation in photonic crystal fiber." Reviews of modern physics 78, no. 4 (2006): 1135.

33. Kano, Hideaki, and Hiro-O. Hamaguchi. "Ultrabroadband $\left(>2500 \mathrm{~cm}^{-1}\right)$ multiplex coherent anti-Stokes Raman scattering microspectroscopy using a supercontinuum generated from a photonic crystal fiber." Applied Physics Letters 86, no. 12 (2005): 121113.

34. Boyd, Robert W. Nonlinear optics. Academic press, 2003.

35. Alfano, R. R., and S. L. Shapiro. "Emission in the region 4000 to $7000 \AA$ via fourphoton coupling in glass." Physical Review Letters24, no. 11 (1970): 584.

36. Stolen, R. H., and Chinlon Lin. "Self-phase-modulation in silica optical fibers." Physical Review A 17, no. 4 (1978): 1448.

37. Brodeur, A., and S. L. Chin. "Ultrafast white-light continuum generation and self- 
focusing in transparent condensed media." JOSA B 16, no. 4 (1999): 637-650.

38. Russell, Philip. "Photonic crystal fibers." science 299, no. 5605 (2003): 358-362.

39. Russell, Philip St J. "Photonic-crystal fibers." Journal of lightwave technology 24, no. 12 (2006): 4729-4749.

40. Agrawal, Govind P. Nonlinear fiber optics. Academic press, 2007.

41. Wadsworth, William J., N. Joly, Jonathan C. Knight, Tim A. Birks, F. Biancalana, and P. St J. Russell. "Supercontinuum and four-wave mixing with Q-switched pulses in endlessly single-mode photonic crystal fibres." Optics express 12, no. 2 (2004): 299309.

42. Available at: http://www.nktphotonics.com/wpcontent/uploads/sites/3/2015/02/nonlinear-fibers.pdf

43. Amiot, Caroline, Antti Aalto, Piotr Ryczkowski, Juha Toivonen, and Goëry Genty. "Cavity enhanced absorption spectroscopy in the mid-infrared using a supercontinuum source." arXiv preprint arXiv:1706.01335 (2017).

44. Available at: http://www.nktphotonics.com/lasers-fibers/en/productcategory/supercontinuum-lasers/

45. Available at: https://www.thorlabs.com/newgrouppage9.cfm?objectgroup_id=5519 


\section{Chapter 2: Fiber Based Cavity Enhanced Absorption Spectroscopy}

In this project, we are trying to build a spectrometer for liquid phase sensing, especially for $\mathrm{H}_{2} \mathrm{O}$ sensing in different liquid solutions. By combining Cavity Enhanced Absorption Spectroscopy (CEAS) with fiber based optical sensing, we are able to take the advantages of both higher sensibility of CEAS and simple but stable liquid sensing using optical fibers. Even though optical loss on fiber connections are usually higher than cavity resonators for gaseous sensing, and there is more loss in fiber transitions to make even less round trips in fiber resonators than conventional cavity resonators, it is still more reliable and easier to implement than simply single-pass sensing or other liquid phase sensing methods. In this chapter we will demonstrate our apparatus in details to show how it works. Optical losses in the device will be discussed and some critical points will be described in building up the whole setup.

\subsection{Fiber-loop Resonator and Supercontinuum in the Resonator}

Since evanescent wave spectroscopy (EWS) is a relatively simple and reliable way to perform optical tests in liquid phase, $[1,2]$ it is expected that some optical fiber sensing method can be implemented in this work. The use of fiber optical measurement has been widely demonstrated in CRDS and CEAS, mostly in the recent decade, in liquid or solid state sensing in many aspects. [3-5] As we stated in Chapter 1, there are multiple ways to setup fiber-based optical sensing elements. Based on how the fiber system is designed, it 
can be separated as open fiber sensing or closed fiber-loop. Based on how the sensing element is designed, there are gaps and cuvettes [6], or continued fiber by using optical waveguides [5] or special fiber components like fiber tapers [7] or side-polished fibers [3]. Each different method has its own advantages and limitations over the degree of difficulty in setup design, complexity in aligning fibers and other optical components, optical loss of the resonator for a moderate ring-down time, and stability and robustness of the whole system.

Among these setup designs, we figured a closed fiber loop with a sensing region fabricated in loop is the best way for our device since it is well developed and is suitable for the sensing elements we intend to use like a fiber taper and a side-polished fiber. More importantly, this design does not require a very large area for the setup and its components are simple: only a few optics and the rest are optical fibers. Thus, after it is fully set and tested, it can be pretty stable because most part of the system is made from fibers and they are fusion spliced together as a whole that is not easy to be messed for additional optical losses. 


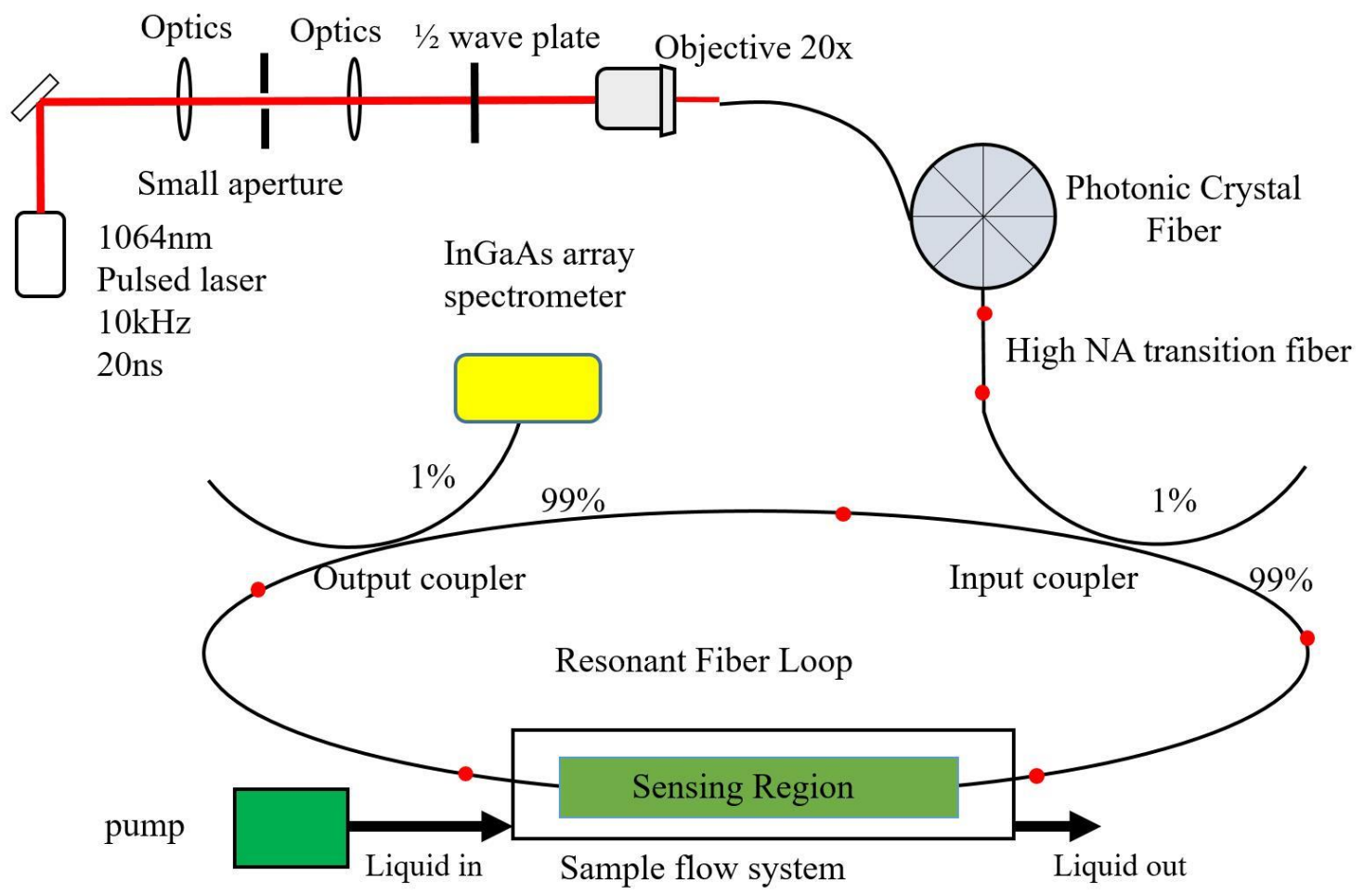

Figure 2.1: Schematic of the supercontinuum fiber-loop cavity enhanced absorption spectroscopy device. Pump laser power ranges from $300 \mathrm{~mW}$ to $1 \mathrm{~W}$ to generate a supercontinuum. The photonic crystal fiber is $\sim 15 \mathrm{~m}$ in length. Red dots stand for fusion splice points.

Figure 2.1 shows a schematic of how our fiber-loop sensing system is designed. The setup can be simply divided into two parts, the Supercontinuum generation and the fiber loop. The supercontinuum generation starts with a Q-switched solid state $\mathrm{Nd}: \mathrm{YVO}_{4}$ laser (Spectra-Physics, T20-8S40-16 laser power supply and V80-106Q laser head) which produces infrared laser at $1064 \mathrm{~nm}$. In order to produce a supercontinuum, the laser is set at pulse mode with a repetition rate of 10 or $20 \mathrm{kHz}$ and a pump power of $300 \mathrm{~mW}$ or more. The laser output is guided by an optical set to get rid of its higher order modes. Its TEM00 mode is then free-space coupled by a 20x microscope to focus on to the input end of the 
15-meter-long Photonic Crystal Fiber (PCF) for supercontinuum generation. The microscope is set in a stage with 5 degrees of freedom, $x-, y-, z$-axis translation and two adjustable angles on $\mathrm{x}$ - and $\mathrm{y}$-axis. The supercontinuum is generated in the PCF, and difference repetition rate and power of the pump laser will give an output supercontinuum different wavelength coverage and power intensity. Figure 1.4 in Chapter 1 is an example of the generated supercontinuum spectra with different pump power. Generally, when the laser is set to work under pulse mode at $20 \mathrm{kHz}$, a significant wavelength broadening effect can be seen with a pump power higher than $300 \mathrm{~mW}$. This power threshold can be even lower at $10 \mathrm{kHz}$.

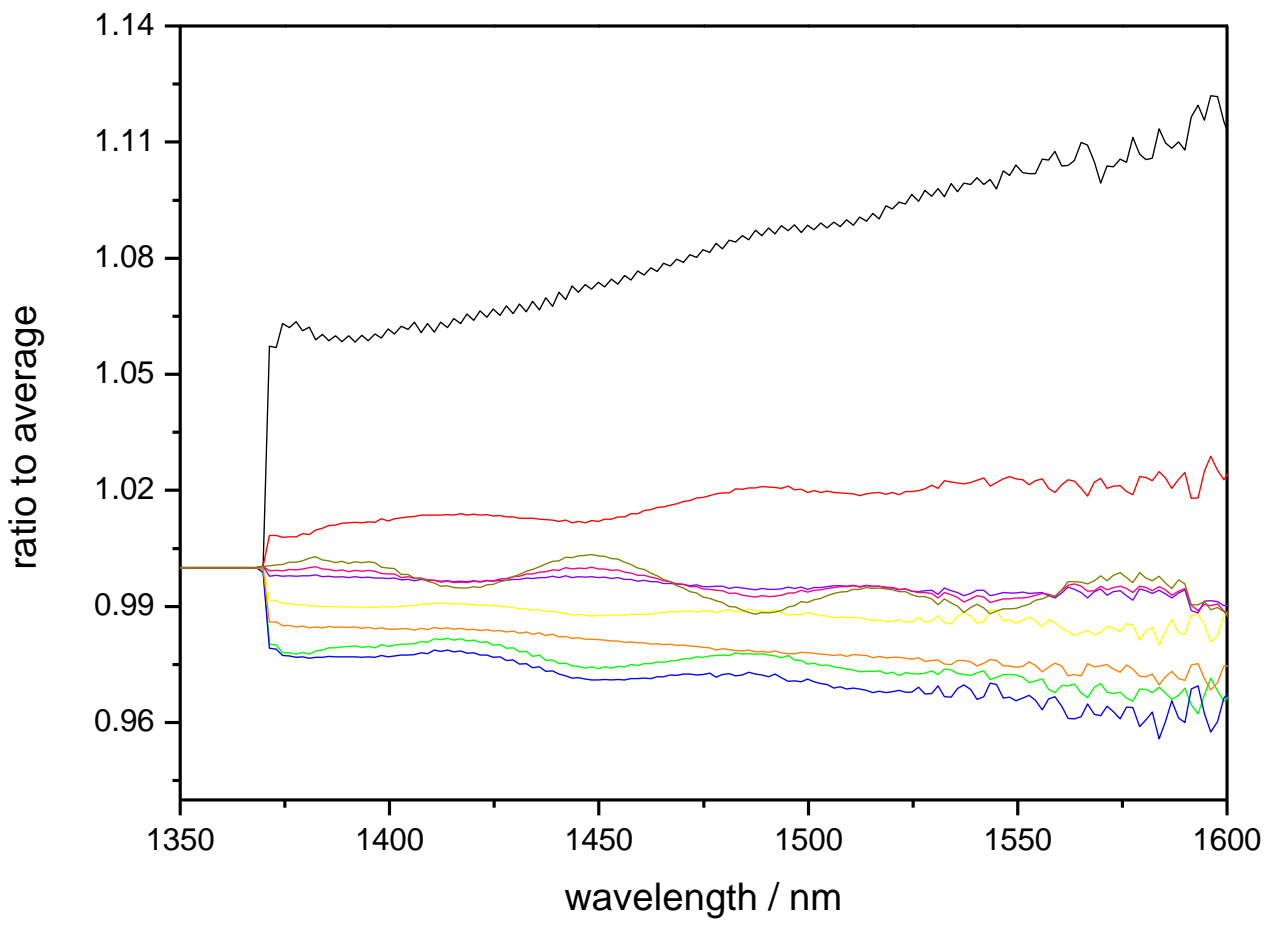

Figure 2.2: Long term stability test of the supercontinuum. Spectra are scanned every hour and ratios are taken against their average. The supercontinuum is seen narrowing during the first two hours of operation. 
The intensity and stability of the generated supercontinuum is an important factor because it is related to the accuracy of the final results. Thus a long term monitoring of supercontinuum intensity is done for each sensor. A supercontinuum is kept running for 810 hours on a same pump power and the spectrometer is scanning the spectra for an empty cavity during this time. The scanned spectra are selected with roughly same time intervals to compare their intensity and wavelength coverage. A ratio is made on each wavelength to show the intensity difference. Figure 2.2 gives a long term test we did on a side-polished fiber sensor. It is easy to find a larger fluctuates (decreases) in light intensity in the first 1.5-2 hours operation. We believe this is mostly coming from the fluctuation of the power of the pump laser. Most of our tests are done after $\sim 1$ hour of running the laser.

The generated supercontinuum is guided into a 500-meter-long fiber-loop by directly splicing the PCF with a single mode fiber. A special high numerical aperture (NA) fiber is used as a transition between the PCF and single mode fiber to reduce the connection loss in the fusion splice of different fibers. [8] With a numerical aperture $(\mathrm{NA}=0.35)$ higher than both a PCF $(\mathrm{NA}=0.20)$ and a single mode fiber $(\mathrm{NA}=0.14)$, it helps to decrease transition loss between different fibers because its high NA allows the supercontinuum from the PCF travelling into it and then out to the single mode fiber as well. A total loss of $\sim 2 \mathrm{~dB}$ can be reached on this point. However, it is still considered to be the highest loss in the whole device, compared with other splice points to be all less than 0.05dB. [9] Two $2 \times 2$ optical couplers with 99/1 splitting ratio are used as input and output on the loop. [10] The couplers 
are fusion spliced on both ends of the single mode fiber and then connected with each other to build a closed loop so that light guided inside can travel in loop multiple times. Each time on the output coupler $1 \%$ of light is guided out to a spectrometer which means $99 \%$ remains inside for multiple passes to enhance the absorption. The spectrometer we use is a NIRQuest 512 spectrometer from the Ocean Optics. It has a spectrum coverage of 900nm to $1700 \mathrm{~nm}$. It is called NIRQuest 512 because it has 512 pixels inside to separate the wavelengths. Thus a resolution of about $1.56 \mathrm{~nm}$ is reached by this spectrometer.

A sensing region is directly fabricated and fusion spliced in the loop. The sensing elements we tested include a fiber taper, a side-polished fiber and a free-space mirror reflection sensor. Details about what they look like and how the sensing work will be further discussed in Chapter 3. A flow system is built around the sensing system for pumping liquid samples into and out of the sensing region. A container is built around the sensors to hold samples and a small pump is connected to pump samples in while we are doing measurements. Different sensor requires different flow system to work. The flow system helps not only in making it easier in adding, changing, or removing samples but also in stabilizing the output spectra as well. Designs of the flow systems for different sensors will be introduced in Chapter 3 together with the sensors.

\subsection{Connection between the PCF and Fiber-loop}

The discussion of this newly designed device starts with the connection between the 
supercontinuum and the fiber-loop. In this device, all other splices in the fiber system are between single mode fibers. By using a fusion splicer (Ericsson, FSU-975) with carefully configured parameters, the connection loss on those points can be made lower than $0.05 \mathrm{~dB}$ ( $1.16 \%$ loss), usually about 0.01 to $0.03 \mathrm{~dB}$. [9] When it comes to different fibers, however, connection losses will be significantly higher than between similar fibers. [11] Our tests show that even a connection between a simple single mode fiber and a multi-mode fiber can have loss as high as several decibels. The main reason for this phenomenon is that the difference in core and cladding diameters for different fibers makes the modes not overlap very well, and different sizes require different temperature on the two fiber ends, so it is more difficult to control the voltage and fusion time used on a fusion splicer. A lower voltage and time makes a splice fragile and easy to break, while a higher voltage and time may cause total destruction on the fiber structure which will cause severe optical loss. 


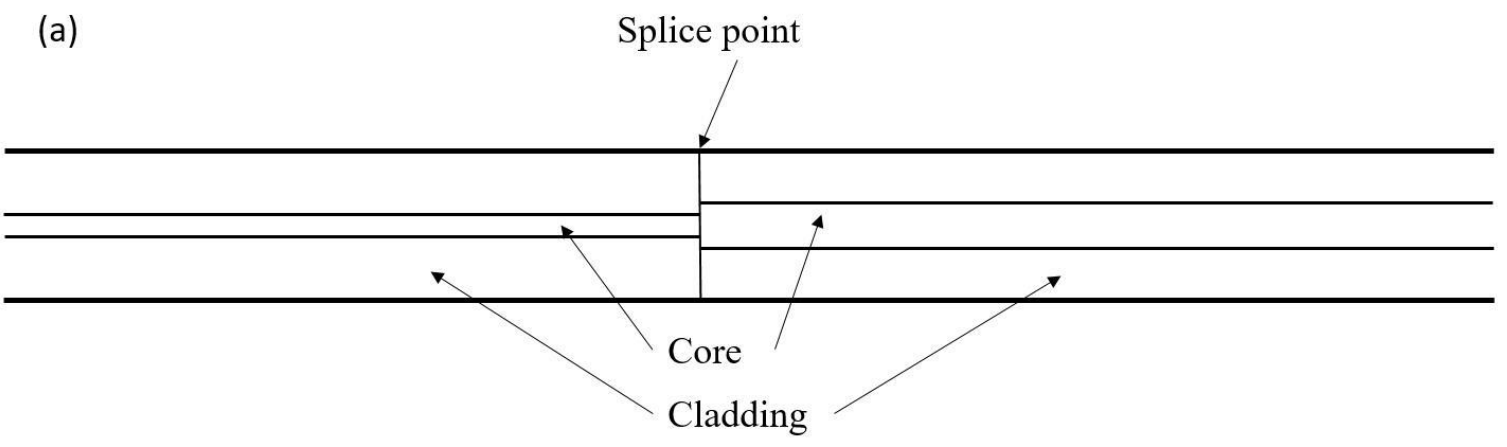

(b)

Tapered transition region

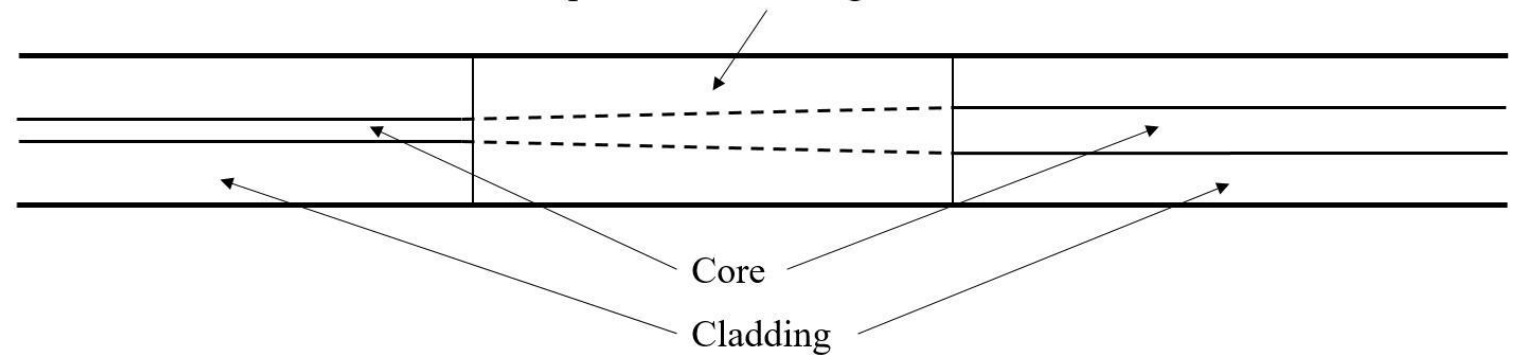

(c)

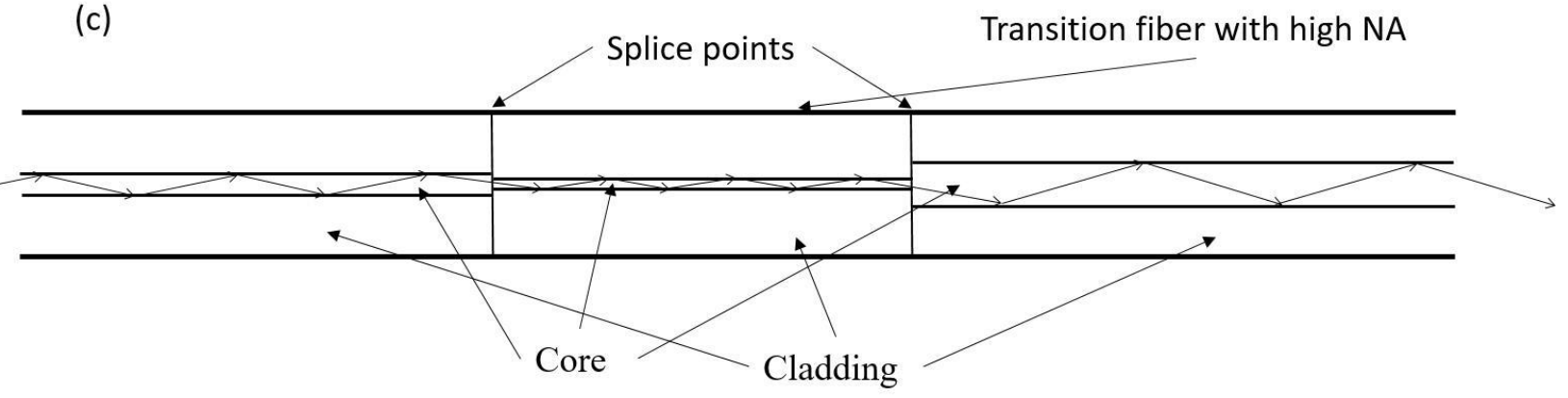

Figure 2.3: Ways of doing connections with different fibers. (a), direct splice. It usually has the highest optical loss. (b), create a taper region from the fiber with larger core size. It requires fine adjustment on splicing parameters and a long time splicing to form the taper. (c), use a transition fiber of a third kind. Several key parameters of the transition fiber we use in this project: core diameter $1.8 \mu \mathrm{m}$, core NA 0.350 , cladding diameter $125.0 \mu \mathrm{m}$. 
Usually there are two solution for this. The first one is to apply a relatively higher time but lower voltage on a fusion splice in order to make a robust splice without damaging the fibers. The second is to build a transition region between the two different size fibers like simply using a transition fiber or building a taper-like transition region from the fiber with larger core diameter or numerical aperture to lower the loss. These methods can help remove most of the connection loss but it is still several times higher than a same-fiber splice. When it comes to a PCF, however, the microstructure of it requires minimum power and time in a fusion splice. [8] Heated at a high temperature, the air holes in a PCF will collapse totally so it can make a worse splicing quality and more importantly, block the light transition inside the fiber to cause a significantly high optical loss. Our trials of directly connection of a single mode fiber with the PCF confirms this phenomenon by giving a loss of higher than $17 \mathrm{~dB}$. The idea of building a transition taper would be not practical as well since it also requires a long time heating and some fiber pulling inside the fusion splicer.

Figure 2.3 gives some schematics of how the connections of different fibers usually work. [12] Figure 2.3(a) and (b) are the connection methods we introduced above of direct connection and transitions using a self-made fiber taper. In a common sense the method in (a) will have a highest loss, which is proved by our tests of a loss of $17 \mathrm{~dB}$ in a splice between a PCF and a single mode fiber. But sometimes with a very fine alignment to make a high mode overlapping, this can have a relatively low loss of $\sim 1$ or $2 \mathrm{~dB}$ [12] since both 
(b) and (c) require two slices and there will be losses on the transitions more or less. Figure 2.3(c) shows the way we use in this project for the connection of the PCF and single mode fiber loop. Trials using method showed in Figure 2.3(a) gives a loss as high as $17 \mathrm{~dB}$ and a loss of more than $10 \mathrm{~dB}$ using method (b). Thus we switched to method (c) trying to find a third kind of fiber as a transition between to lower the connection loss. Several different fibers are tested including single mode fibers with different core sizes, multi-mode fibers, and other fibers with different properties. Our final choice is a high numerical aperture fiber purchased from Nufern (UHNA3, Ultra-High NA Select Cutoff Single-Mode Fiber). We select this fiber mainly because of its high numerical aperture and small core size. A numerical aperture of a fiber corresponds with the output angle of the light beam. A higher numerical aperture means a wider angular range the output light from the fiber can cover and the light coming inside this angle range can come inside as well. The numerical aperture of this UHNA3 fiber is higher than both a PCF and a single mode fiber even with a thinner core, so we can make sure that the whole beam of supercontinuum coming out from a PCF can go through it and then go to the single mode fiber as well. In this way we are able to minimize the loss on transitions from one fiber to another. This helps reducing connection loss on fibers with different sizes significantly. Another reason we choose this UHNA3 is that it has a very small core size with a diameter of only $1.8 \mu \mathrm{m}$. The small diameter makes the fusion splice process take less time and power to complete. Some changes are made on splicing parameters of our fusion splicer, aiming at minimizing splicing voltage and fusion time to preserve the micro air holes in the PCF as intact as 
possible. Some key parameters of the fusion splices between the different fibers are listed in Table 2.1, compared with the preset parameters for standard single mode-singe mode splice. If the air holes do not collapse, there will be no unexpected fiber materials to block supercontinuum transmission, so a minimum loss can be reached. With some adjustments on splicing parameters, we make the connection loss to reach about $2 \mathrm{~dB}$ on this connection.

Table 2.1: Splicing parameters on PCF-UHNA3 and UHNA3-single mode fiber (SM)

\begin{tabular}{|c|c|c|c|}
\hline & SM - SM & SM - UHNA3 & PCF - UHNA3 \\
\hline Prefusion time $^{1}$ & $0.2 \mathrm{~s}$ & $0.2 \mathrm{~s}$ & $0.2 \mathrm{~s}$ \\
\hline Prefusion current & $10.0 \mathrm{~mA}$ & $10.0 \mathrm{~mA}$ & $7.0 \mathrm{~mA}$ \\
\hline Gap & $50.0 \mu \mathrm{m}$ & $50.0 \mu \mathrm{m}$ & $50.0 \mu \mathrm{m}$ \\
\hline Overlap $^{2}$ & $10.0 \mu \mathrm{m}$ & $10.0 \mu \mathrm{m}$ & $3.0 \mu \mathrm{m}$ \\
\hline Fusion time 1 & $0.3 \mathrm{~s}$ & $0.3 \mathrm{~s}$ & 0s \\
\hline Fusion current 1 & $10.5 \mathrm{~mA}$ & $10.5 \mathrm{~mA}$ & $0 \mathrm{~mA}$ \\
\hline Fusion time 2 & $2.0 \mathrm{~s}$ & $2.0 \mathrm{~s}$ & $1.8 \mathrm{~s}$ \\
\hline Fusion current 2 & $16.3 \mathrm{~mA}$ & $16.3 \mathrm{~mA}$ & $10.0 \mathrm{~mA}$ \\
\hline Fusion time 3 & $2.0 \mathrm{~s}$ & $2.0 \mathrm{~s}$ & Os \\
\hline Fusion current 3 & $12.5 \mathrm{~mA}$ & $12.5 \mathrm{~mA}$ & $0 \mathrm{~mA}$ \\
\hline Left MFD ${ }^{3}$ & $9.8 \mu \mathrm{m}$ & $3.2 \mu \mathrm{m}$ & $4.8 \mu \mathrm{m}$ \\
\hline Right MFD & $9.8 \mu \mathrm{m}$ & $9.8 \mu \mathrm{m}$ & $3.2 \mu \mathrm{m}$ \\
\hline AOA current ${ }^{4}$ & $0 \mathrm{~mA}$ & $0 \mathrm{~mA}$ & $10 \mathrm{~mA}$ \\
\hline
\end{tabular}

1. Prefusion is a procedure to use a rapid arc charge to clean the fiber ends.

2. Overlap means the overlap of the two fibers in a splice. It is smaller for PCFs so the micro structures can be kept intact.

3. MFD is the mode field diameter, which is set the same as the MFD of the fiber inserted. 4. AOA is short for arc-on alignment, which is for stabilizing the splice loss for fibers with smaller core diameters. 


\subsection{Theoretical and Experimental Loss of the Fiber-loop Resonator}

The loss on the connection between PCF and single mode fiber is important because we want to guide as much supercontinuum into the fiber-loop as possible. However since it is only a single-time pass, what we care more is still the optical loss inside the loop because it directly correspond with the ring-down time we can have with the loop resonator, or in CEAS, the number of round trips the supercontinuum can make inside the loop. In order to get a rough estimation of our new device, some calculations and tests are done before making it work for liquid samples. Table 2.2 shows some typical ranges of optical loss on every optical components we have in loop. In each round trip, we will have 500 meters of single mode fiber, 2 passes on optical couplers, 5 fusion splice points and a single pass on the sensing element. The total loss can be written as:

$$
L=2 L_{\text {coupler }}+5 L_{\text {splice }}+0.5 L_{\text {fiber }}+L_{\text {sensor }}+L_{\text {absorbance }}
$$

Because the mirror reflection sensor has a relatively high transition loss on free space coupling light from one fiber to another, our calculation here are mainly based on a sidepolished fiber sensor.

Table 2.2 Typical optical loss on fiber-loop components

\begin{tabular}{|l|c|c|c|c|}
\hline & Fiber coupler & Optical fiber $/ \mathrm{km}$ & Fusion splice & Fiber sensor \\
\hline Insertion Loss (dB) & 0.04 & $0.19-0.50$ & $0.01-0.05$ & 0.20 \\
\hline Insertion Loss (\%) & 1 & $4.3-10.8$ & $0.23-1.16$ & 4.71 \\
\hline
\end{tabular}


According to the table and equation above, a calculated estimation of a round trip loss in loop for an empty cavity should be in a range of 0.43 to $0.78 \mathrm{~dB}$, or $10.4 \%$ to $19.7 \%$. Compared with a gas phase resonant cavity, this round trip loss is much higher which means the finesse of our fiber-loop resonator will be much smaller than a conventional gas phase cavity. Instead of making hundreds or thousands of round trips, it can only make several round trips in this fiber-loop. A low enhancement factor is a limitation liquid phase CEAS has over gas phase, but it still gives times of enhancement compared to a single pass device.

Tests are done after the estimation of round trip loss to show how the system works. In Chapter 1 we have the ring-down time calculated as:

$$
\tau(v)=\frac{d}{c[(1-R)+\alpha(v) d]}
$$

The denominator is a round trip loss in a resonator so in this work it can be simply written as:

$$
\tau(v)=\frac{d}{c L(v)}
$$

Where $\mathrm{L}$ is the total round trip loss we listed above and $\mathrm{c}$ is the speed of light inside the fiber. So if a ring-down time can be measured, it will be easy to get a round trip loss together with an effective number of round trips on each wavelength. 
(a)
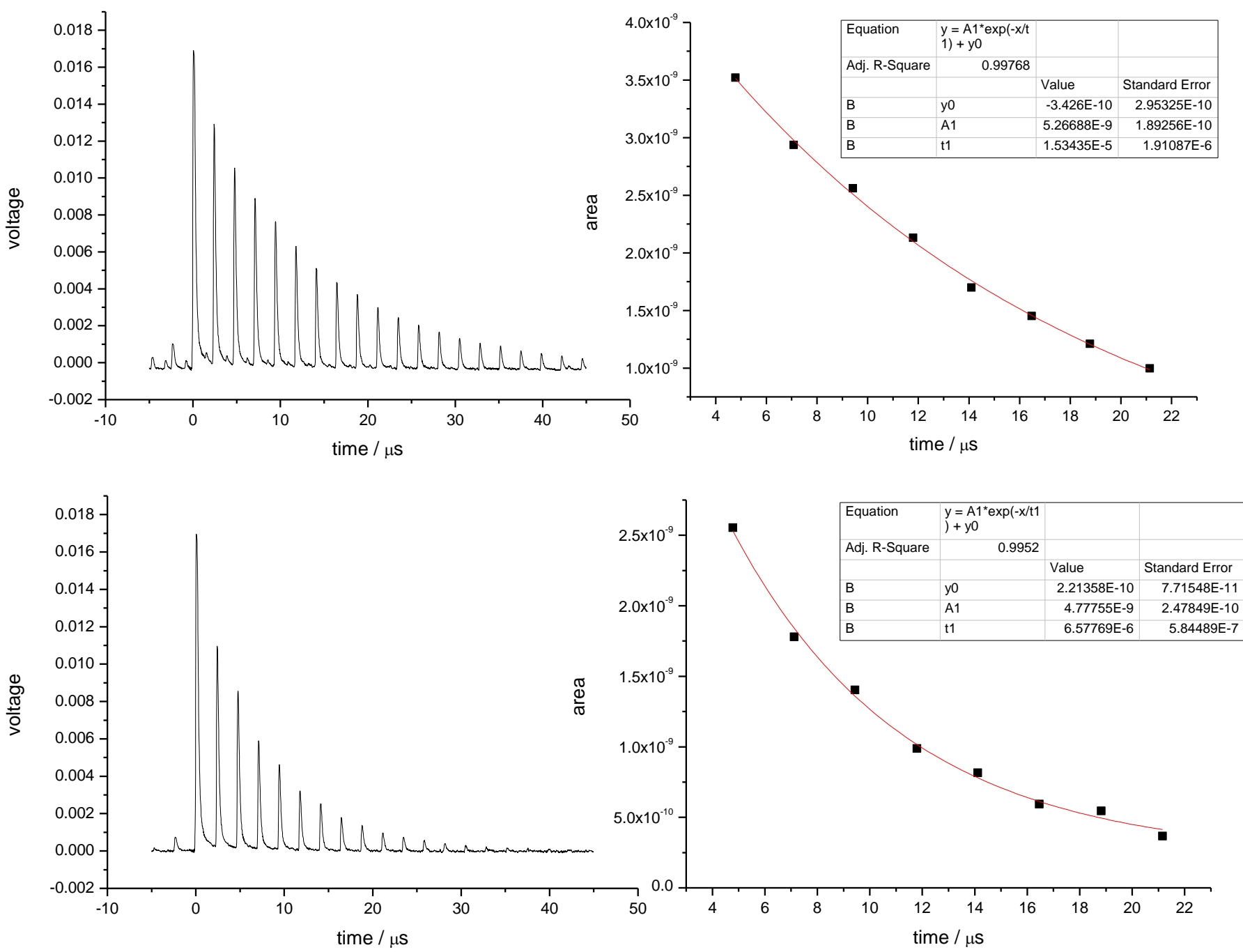
(b)

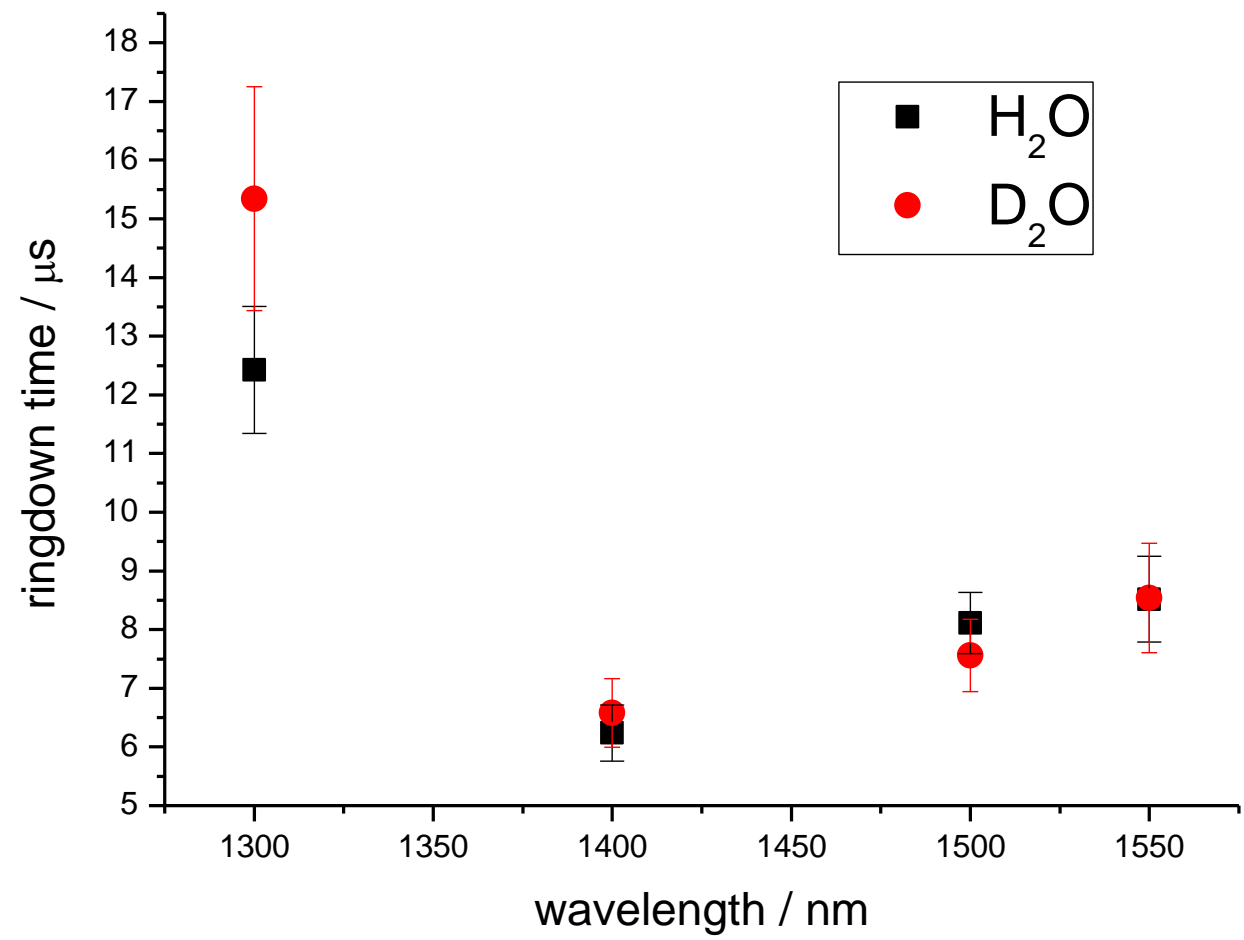

Figure 2.4: Ring-down data and ring-down time fit for $1300 \mathrm{~nm}$ and $1400 \mathrm{~nm}$ for $\mathrm{D}_{2} \mathrm{O}$. Areas are fit from the second peak to avoid influence from light coupled into cladding modes. A comparison of all ring-down time in the region of 1000 to $1600 \mathrm{~nm}$ is also attached in the bottom figure.

Several interference filters [13] are used to separate one wavelength each time to do a ringdown time measurement. Figure 2.4 (a) shows several ring-down time decays we fitted for the wavelength of $1300 \mathrm{~nm}$ and $1400 \mathrm{~nm}$ respectively, and more ring-down time data is plotted in Figure 2.4(b). We are testing both $\mathrm{H}_{2} \mathrm{O}$ and $\mathrm{D}_{2} \mathrm{O}$ samples because $\mathrm{H}_{2} \mathrm{O}$ has an $\mathrm{O}-$ $\mathrm{H}$ bond absorption at $1450 \mathrm{~nm}$ but $\mathrm{D}_{2} \mathrm{O}$ does not, so our main interest lies in the near infrared region of 1300 to $1500 \mathrm{~nm}$. Therefore pure $\mathrm{D}_{2} \mathrm{O}$ sample can work as a reference. The round trip loss lies between $16 \%$ and $22 \%$ in this region, which is slightly higher than our estimation. Possible reasons are the loss on optical fiber is slightly higher than what we 
listed in table 2.2, and loss on a side-polished fiber can also be higher than what is listed.

For $\mathrm{H}_{2} \mathrm{O}$ and $\mathrm{D}_{2} \mathrm{O}$ the ring-down times are similar so we can directly compare their output intensities when we are trying to get an absorbance on $\mathrm{H}_{2} \mathrm{O}$ in neglect of the difference in the number of round trips.

\subsection{Noise Level and Sensitivity}

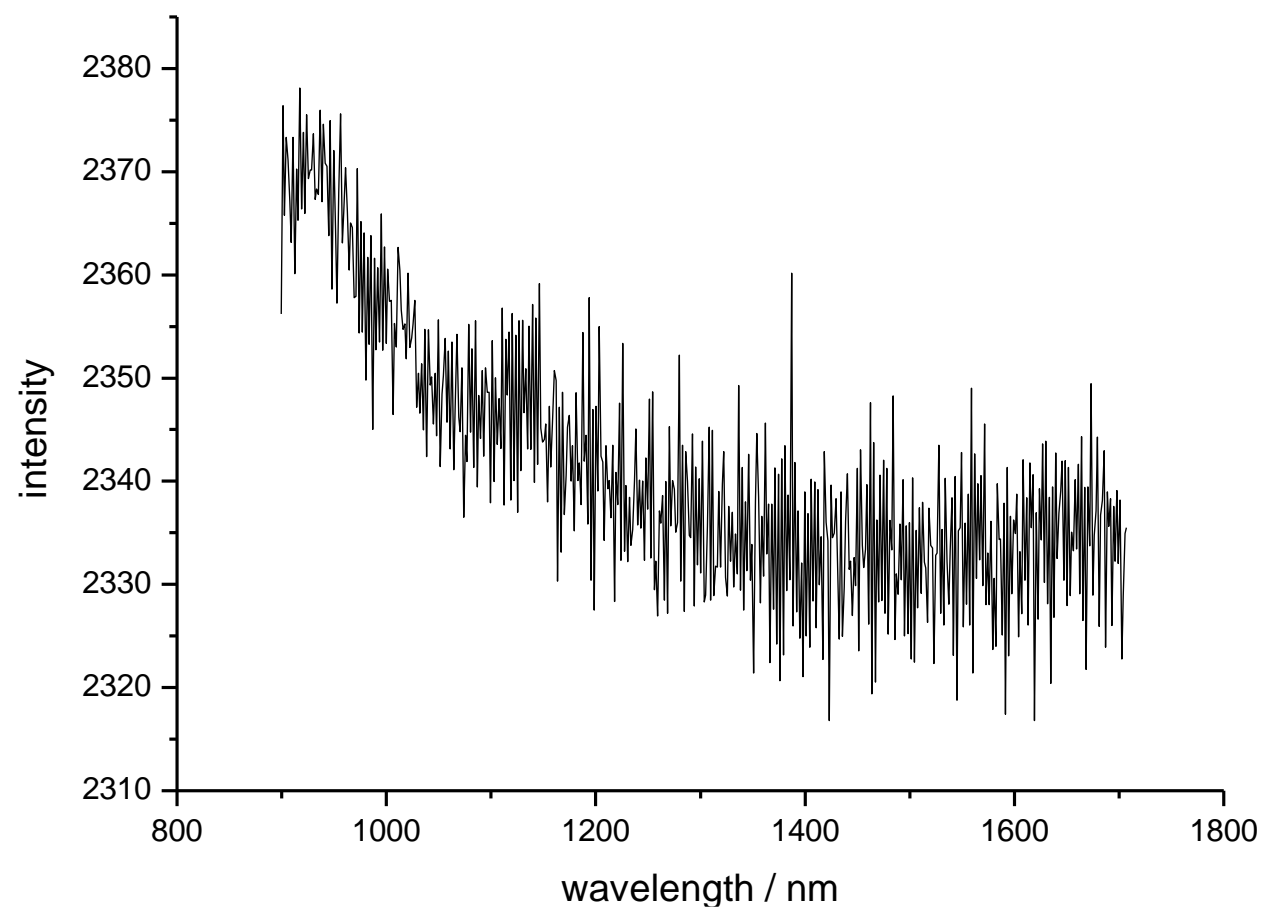

Figure 2.5: background (dark) noise of the spectrometer in this project at an integration time of $20 \mathrm{~ms}$ and averaging of 100 times, which is similar as an integration time usually used in acquiring sample spectra.

Theoretically, the minimum noise level we can get is the same as a dark spectrum measured from the spectrometer. Figure 2.5 shows the baseline of the background noise we have on 
the dark spectrum. The intensity on the $y$-axis is only a relative number and its saturation point is 66000 . If we estimate the signal level to be relatively around 50000 to 60000 in intensity we can have a background noise level of $0.03 \%$ of the signal. Further experiments on empty cavity (simply air in the sensing region) confirms our estimation.

When samples are added into the system, extra noise is observed in the spectra. The noise features for different sensors are not the same, the mirror reflection sensor is observed to have a higher noise level than the other two, especially when testing on organic samples. Further discussions on noise levels and signal to noise ratios will be demonstrated in Chapter 4 when the experimental results are demonstrated.

\subsection{Conclusions}

In this Chapter we have demonstrated the design of our liquid phase Cavity enhanced absorption device and discussed its feasibility. The device we build made a combination of a supercontinuum generation system with a fiber-loop resonator to take the advantages of both. A broadband high intensity supercontinuum light is one of the best among all the broadband light sources and the simple but robust fiber-loop cavity makes the whole liquid phase sensing system inexpensive and reliable as well. The use of a PCF as the supercontinuum generation medium makes it possible to use a relatively low power laser source as the pump. A minimum requirement of only $300 \mathrm{~mW}$ is enough for a moderate wavelength broadening and the whole coverage of this supercontinuum can reach more 
than $1000 \mathrm{~nm}$ (ranging from 600 to $1600 \mathrm{~nm}$ ) when the pump power is $1 \mathrm{~W}$. A ring-down time is also fitted for several different wavelengths. An exponential fit indicates that this supercontinuum does make multiple round trips inside the resonator. Although the number of round trips cannot match with what we can usually have with a gas phase resonator, it gives several times of enhancement in the signal level. Theoretical estimation matches with experimental results to give $\sim 20 \%$ loss per round trip near $1400 \mathrm{~nm}$ to $1500 \mathrm{~nm}$. The formation of a fiber-loop from a long $(500 \mathrm{~m})$ single mode fiber make a ring-down relatively long so we could record a ring-down signal precisely to fit the decay. Finally some noise level measurements are done on this device. For a side-polished fiber sensor the noise level is reasonable for some experiments but it grows when we switch to a mirror reflection system, especially for organic samples. We have made some efforts to get rid of most of the noise so far, but it is still several times higher than a side-polished fiber sensor.

\subsection{References}

1. Wolfbeis, Otto S. "Fiber-optic chemical sensors and biosensors." Analytical chemistry 80, no. 12 (2008): 4269-4283.

2. Pickrell, Gary, Wei Peng, Bassam Alfeeli, and Anbo Wang. "Fiber optic chemical sensing." In Optics East 2005, pp. 59980D-59980D. International Society for Optics and Photonics, 2005.

3. Waechter, Helen, Jessica Litman, Adrienne H. Cheung, Jack A. Barnes, and Hans-Peter 
Loock. "Chemical sensing using fiber cavity ring-down spectroscopy." Sensors 10, no. 3 (2010): 1716-1742.

4. Waechter, Helen, Dorit Munzke, Angela Jang, and Hans-Peter Loock. "Simultaneous and continuous multiple wavelength absorption spectroscopy on nanoliter volumes based on frequency-division multiplexing fiber-loop cavity ring-down spectroscopy." Analytical chemistry 83, no. 7 (2011): 2719-2725.

5. Bescherer, Klaus, Jack A. Barnes, and Hans-Peter Loock. "Absorption measurements in liquid core waveguides using cavity ring-down spectroscopy." Analytical chemistry 85, no. 9 (2013): 4328-4334.

6. Van der Sneppen, L., F. Ariese, C. Gooijer, and W. Ubachs. "Liquid-phase and evanescent-wave cavity ring-down spectroscopy in analytical chemistry." Annual Review of Analytical Chemistry 2 (2009): 13-35.

7. Tarsa, Peter B., Aislyn D. Wist, Paul Rabinowitz, and Kevin K. Lehmann. "Single-cell detection by cavity ring-down spectroscopy." Applied Physics Letters 85, no. 19 (2004): 4523-4525.

8. Xiao, Limin, M. S. Demokan, Wei Jin, Yiping Wang, and Chun-Liu Zhao. "Fusion splicing photonic crystal fibers and conventional single-mode fibers: microhole collapse effect." Journal of lightwave technology 25, no. 11 (2007): 3563-3574.

9. Marcuse, Dietrich. "Loss Analysis of Single-Mode Fiber Splices." Bell Labs Technical Journal 56, no. 5 (1977): 703-718.

10. Brown, R. Stephen, Igor Kozin, Zhaoguo Tong, Richard D. Oleschuk, and Hans-Peter 
Loock. "Fiber-loop ring-down spectroscopy." The Journal of chemical physics 117, no. 23 (2002): 10444-10447.

11. Bechamps, Ronald D., and Wayne L. Peterson. "Apparatus for storing and splicing optical fibers." U.S. Patent 5,966,492, issued October 12, 1999.

12. Yablon, Andrew D. Optical fiber fusion splicing. Vol. 103. Springer Science \& Business Media, 2005.

13. "Infra-red interference filter." U.S. Patent 3,682,528, issued August 8, 1972. 


\section{Chapter 3 Liquid Phase Sensing Theories and Methods}

As stated in Chapter 2, several different sensing elements are tried in this project, including fiber tapers, side-polished fibers, and a mirror reflection sensor. Each sensor has its own advantages but also limitations as well. In this chapter we will discuss the theory of each fiber sensor of how it works and how to make it inside the loop system. We will focus mostly on the sensors themselves in this chapter about their stability, sensitivity and some noise level discussion. Further experimental results on different samples will be demonstrated in Chapter 4. The flow system designs are different for different sensors but all aiming at making the change of samples easier.

\subsection{Fiber Tapers}

A fiber taper, or a biconical tapered fiber, is a fiber component commonly used in telecommunication and optical sensing. [1] In a CRDS or CEAS device, a taper is used for its relatively high evanescent field intensity [2] which means more light in the resonator can be used in sample sensing. In evanescent wave spectroscopy, because only the evanescent field interacts with samples, the proportion of light source working as evanescent field is an important character we need to think about, which is different from a gas phase sensing where a whole light beam is in use when it is introduced into a resonant cavity. 
(a)

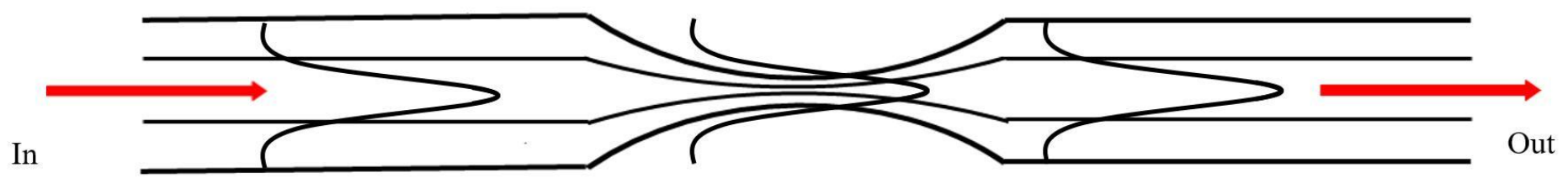

Tapered region

(b)

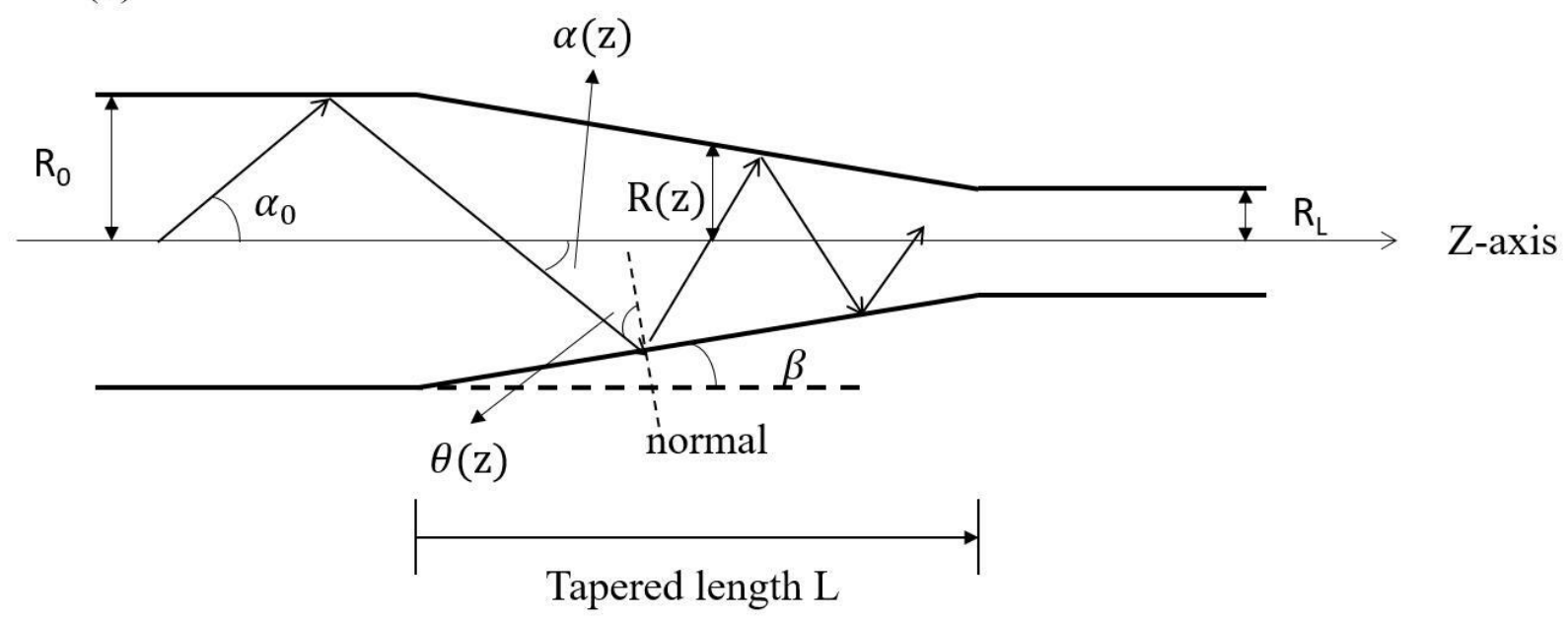

Figure 3.1: Schematics of a fiber taper. (a), what an optical fiber taper looks like and how the evanescent field is enhanced on a taper. The narrowed region is the tapered region and the narrowest part is called the waist of a taper. (b), a diagram of the core of a single mode fiber taper for the illustration of the penetration depth calculations below. It is imagined to be a linear taper only to simplify the calculation. Other taper shapes (parabolic, exponential, etc.) have the same calculation process only with more complex expressions.

Figure 3.1(a) shows a schematic of what a taper looks like, and it is simple to figure out that it is in the waist region that we are getting the maximum evanescent field. Some key characters are demonstrated in Figure 3.1(b) as we are determining the penetration depth and field intensity of an evanescent wave. [3] With a simple optical fiber, the penetration 
depth is usually determined as:

$$
d_{p}=\frac{\lambda}{2 \pi \sqrt{n_{1}^{2} \sin ^{2} \theta-n_{2}^{2}}}
$$

Where $\lambda$ is the wavelength of incident light, $n_{1}$ and $n_{2}$ are the refractive index of the core and cladding of the fiber, $\theta$ is the incident angle $\left(\frac{\pi}{2}-\alpha\right)$. For a simple linear optical fiber taper showed in Figure 3.1(b) [3], we define radius of the taper R(z) with position $\mathrm{z}$ on the taper and it decreases from original fiber radius $\mathrm{R}_{0}$ to the size of taper waist $\mathrm{R}_{\mathrm{L}}$ with a total tapered length L. Also $\alpha(\mathrm{z})$ is the launch angle at position $\mathrm{z}$ with initial angle of $\alpha_{0}$, and $\theta(\mathrm{z})$ is the incident angle at position $\mathrm{z}$. The radius and launch angle has a relation of:

$$
R_{0} \sin \left(\alpha_{0}\right)=R(z) \sin \left(\alpha_{z}\right)
$$

For a linear fiber taper, the radius can be expressed as:

$$
\mathrm{R}(\mathrm{z})=\frac{Z}{L}\left(R_{0}-R_{L}\right)+R_{L}
$$

So we have the angle at $\mathrm{z}$ to be:

$$
\alpha_{z}=\arcsin \left[\frac{\sin \left(\alpha_{0}\right)}{\left(1-\frac{R_{L}}{R_{0}}\right) \frac{Z}{L}+\frac{R_{L}}{R_{0}}}\right]
$$

The incident angle $\theta$ is related to $\alpha_{z}$ and the taper angle $\beta$ as well:

$$
\begin{gathered}
\theta(\mathrm{z})=\frac{\pi}{2}-\alpha_{z}-\beta \\
\beta=\arctan \left(\frac{R_{0}-R_{L}}{L}\right)
\end{gathered}
$$

With the numerical aperture (NA) of a fiber to be:

$$
\mathrm{NA}=\sqrt{n_{1}^{2}-n_{2}^{2}}=\sin \left(\theta_{c r t}\right)
$$

Where $\theta_{c r t}$ is the critical angle for total internal reflection, we can have the penetration 
depth for a fiber taper to be:

$$
d_{p}=\frac{\lambda \sqrt{1-\frac{n_{a q}^{2}}{n_{c}}}}{2 \pi N A \sqrt{\cos ^{2}\left\{\arcsin \left[\frac{\alpha_{0}}{\left(1-\frac{R_{L}}{R_{0}}\right)^{2}} \frac{Z}{L}+1\right]+\arctan \left(\frac{R_{0}-R_{L}}{L}\right)\right\}-\left(\frac{n_{a q}}{n_{c}}\right)^{2}}}
$$

Where we change $n_{1}$ and $n_{2}$ to $n_{c}$ for the refractive index of the fiber core and $n_{a q}$ for the refractive index of surrounding liquid. Here the cladding index is ignored since we assume cladding on a taper is thin enough for the core to make direct contact with samples.

From the equation above, it is not difficult to find when an optical fiber taper is made, the penetration depth is determined, which means at a certain wavelength, penetration depth will be certain for a certain taper. Excluded for some predetermined characters like the refractive index of a fiber core and liquid sample along with the fiber size, the penetration depth at a certain position $\mathrm{z}$ on a taper is mainly depend on two numbers: the final taper waist $R_{L}$ and the initial angle $\alpha_{0}$. Calculations of different fiber shapes like a parabolic or exponential taper will be more complex but with similar results. [3]

Based on Equation (3.8), the narrower the waist $R_{L}$ and the smaller in the incident angle $\theta$ (or larger on the initial angle $\alpha_{0}$ ), the more evanescent field penetrates. Some other work has already been done with manufactured fiber tapers previously in our group, $[4,5]$ so in this project we are working on making our own tapers to do the job. 


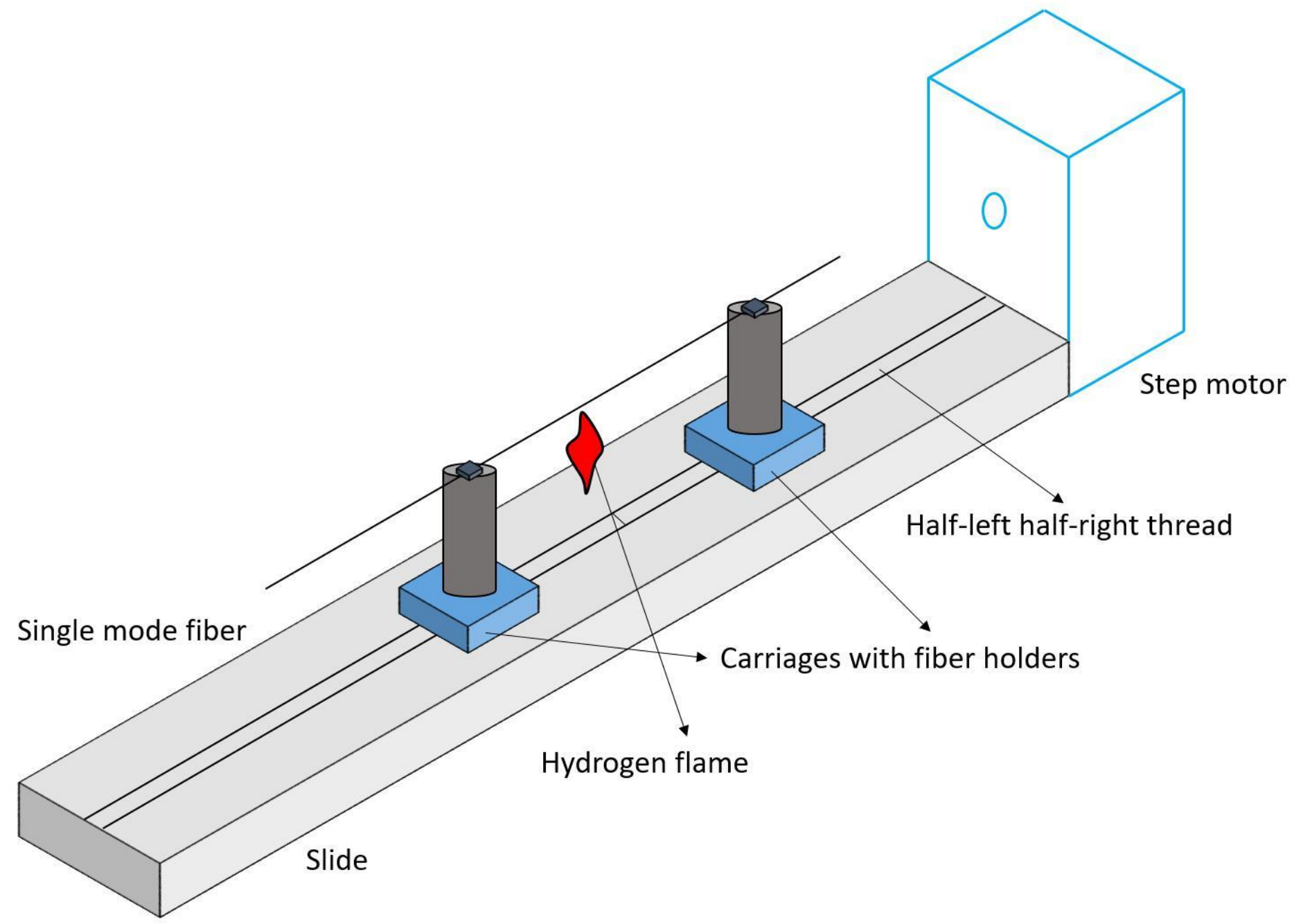

Figure 3.2: Schematic of the fiber pulling device. The flame is coming from a hydrogen torch and the heated single mode fiber has its coating removed, leaving only core and cladding.

Almost all works of making tapers are done by heating and pulling a bared single mode fiber. [2, 6] In this work we build our pulling system out from a slide with an electrical step motor (Velmex Unislide, 12.5") and a hydrogen torch (Premier Industries, 3-H Hydrogen/Oxygen torch). As shown in Figure 3.2, there are two carriages on the slide, attached on either side of a left-right screw in the slide. When the motor is on, it can drive 
the screw to make the two carriages go on the opposite directions simultaneously. A pole with a fiber clamp is fixed on each carriage to hold a fiber over the slide. The resolution of this slide is $0.050 \% / \mathrm{rev}$, and the motor is $200 \mathrm{steps} / \mathrm{rev}$, which makes the total resolution of the slide to be $2.5 \times 10^{-4 "} /$ step. A total of 2000 steps can make a separation of 1 " in a time period of $\sim 2 \mathrm{~min}$. A bared single mode fiber is held by the two clamps on the carriages when the motor is turned on and the torch is lit at the same time. We choose a hydrogen torch because the melting points for both fiber core and cladding (silica, $1713^{\circ} \mathrm{C}$ ) [7] are high and only a hydrogen flame $\left(2254^{\circ} \mathrm{C}\right.$ compared with a $\mathrm{CH}_{4}$ flame of only $\left.1963^{\circ} \mathrm{C}\right)$ [8] can easily reach a temperature high enough to make it soft and reshaped. The heated fiber is pulled to become a taper. Pulling time is set to be $2 \mathrm{~min}$ and hydrogen and oxygen flow rates are controlled to have a flame high enough to cover the fiber. A total length of 15$18 \mathrm{~mm}$ is obtained for a taper each time. Because the flame cannot stay exactly the same each time, the taper length and shape are not always the same.

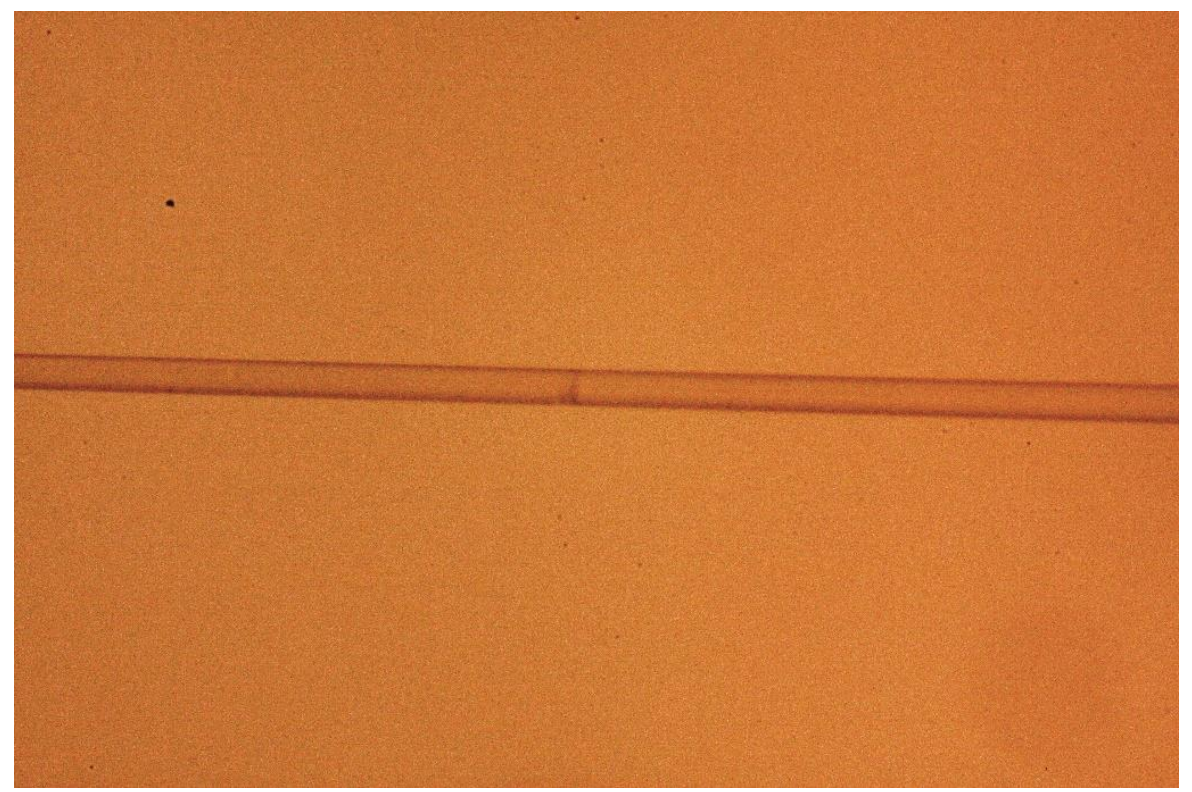




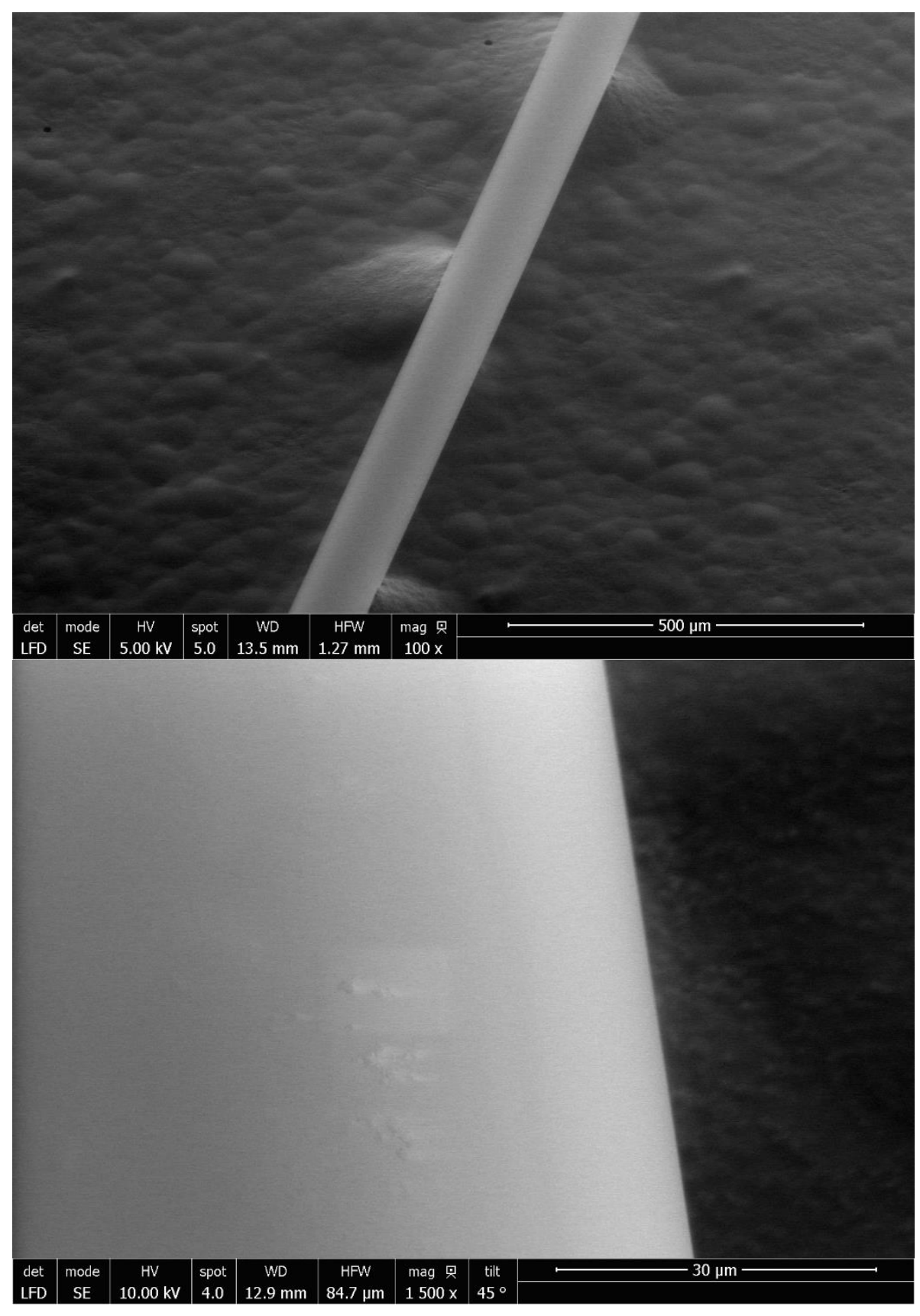

Figure 3.3: images of fiber tapers. The top image is taken from a microscope and the bottom two are taken from the SEM. Most tapers are with a length of $\sim 1.8 \mathrm{~cm}$ and waist of $\sim 20-30 \mu m$. 


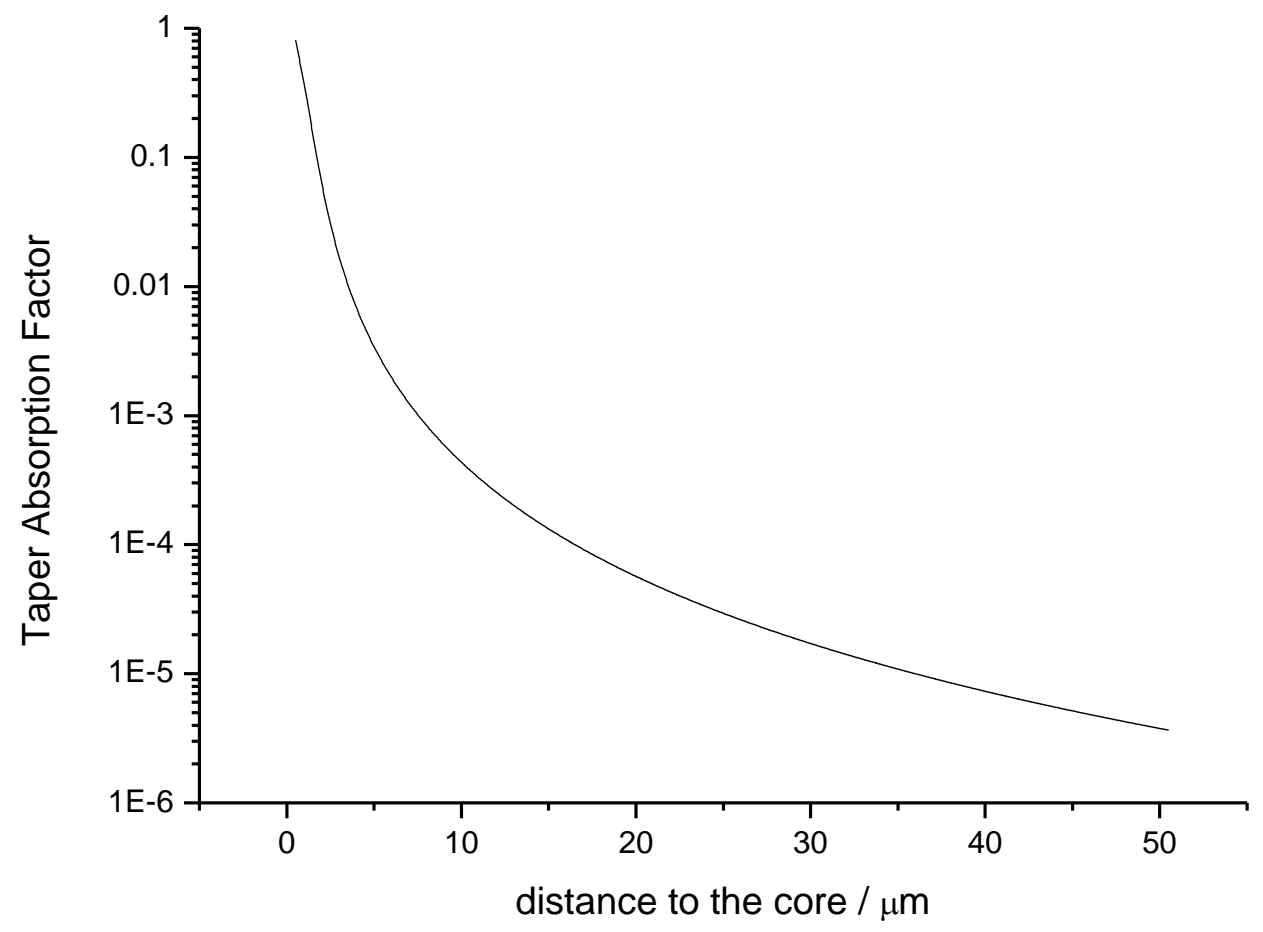

Figure 3.4: Taper absorption factor $\left(\eta_{1} / v_{g}\right)$ versus distance to the surface of the fiber core. Detectable intensity is restricted within several microns around the core.

In order to select a suitable fiber taper for the experiment, we take scanning electron microscope (SEM) images of the tapers and choose the ones with relatively narrower waist size. Figure 3.3 shows some images we have on different tapers and SEM imaging makes it easy to find out difference in their waist sizes. Based on Figure 3.1 and ref[2, 3], The narrower a taper waist is, the more evanescent field there is outside the fiber. The intensity decreases significantly with the distance from the fiber. In terms of evanescent field intensity only, a thinner taper waist is always the better. A schematic of evanescent field absorption factor (Evanescent field energy fraction $\left(\eta_{1}\right)$ divided by group velocity $\left(v_{g}\right)$ of the light propagating in the fiber.) versus the distance to fiber core is shown in Figure 3.4 
[9]. The closer to the core, the more evanescent field can be used, and more evanescent intensity means a higher signal to noise for higher sensitivity.

A narrower waist means higher evanescent field percentage, but it means the taper is more fragile as well. A taper thin enough can be coiled up to a really small radius [10] but only a small twist or a pull along the fiber will make it break into two halves. Another limitation is a taper has to be adiabatic to prevent the light inside to get from core mode to cladding mode [11] or other modes that could make extra noise on our signal. Light in cladding modes is easier to be absorbed by surround samples than in core mode, and going into cladding mode usually means most of it cannot be coupled back to core mode again when coming out of the tapered region. What's more, the light coupled back will have different path length from the light stays in core mode, which will cause interference to give higher noise level. Both situations mean more optical loss and higher noise on a taper than simply an absorption, which makes the final spectra not as stable as expected. We managed to get some results on $\mathrm{H}_{2} \mathrm{O} / \mathrm{D}_{2} \mathrm{O}$ sample pairs with a taper sensor, but most data we get is with very high noise levels.

The self-made taper is also not thin enough to give very high signal level. We believe the self-built pulling system is the main cause for this issue. A more accurate control is required in the pulling process especially in controlling the flame size and position. Uncertainties like unexpected wind in the pulling process and $\mathrm{H}_{2} / \mathrm{O}_{2}$ flow rate instability are in need to 
be monitored and controlled more precisely. Flame temperature is also affected by these uncertainties. A lower temperature usually means the fiber is not soft enough to be pulled so it will not form a taper or even be broken during the process. A higher temperature makes it too soft so a fiber may be not adiabatic or even melt down.

\subsection{Side-polished Fibers}

A side-polished fiber is a fiber component also works by making use of evanescent wave spectroscopy. [12, 13] Its working theory is similar as a fiber taper: exposing the core of a fiber outside by make the cladding thinner. Figure 3.5 is a schematic of what a side-polished fiber looks like from the side. Instead of making the whole region thinner as a fiber taper, a side-polished fiber is fabricated by simply polishing a layer of the cladding out from one side of a single mode fiber. Usually the cladding is removed to leave only several microns away from the core so enough evanescent field can be exposed and reacted with surrounding environment. The other region of the fiber will remains intact which means even in the side-polished region, only less than $50 \%$ of the fiber diameter is removed to leave a reasonably thick part on the fiber. For example, for a typical single mode fiber with $125 \mu \mathrm{m}$ cladding diameter and $9.8 \mu \mathrm{m}$ core diameter, even only $2 \mu \mathrm{m}$ is left on cladding to the core, the side-polished region can still be $\sim 70 \mu \mathrm{m}$ in thickness. Compared with a taper, a much thicker sensing region makes a side-polished fiber more robust and is much easier to handle. This makes it convenient to put a side-polished fiber into the flow system we build and not to worry about breaking. A side-polished fiber is fabricated with very 
dedicate procedures, so the ones we use in this project are purchased from Phoenix Photonics. The polished region is $17 \mathrm{~mm}$ long and the polished depth is $\sim 1-2 \mu \mathrm{m}$ to the core.

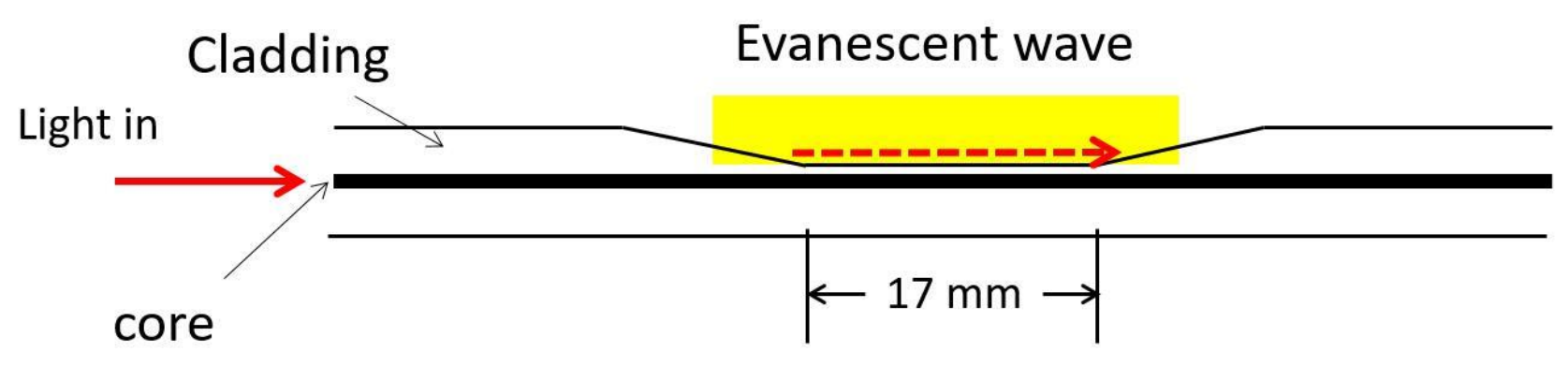

Figure 3.5: A diagram of a side-polished fiber, and the evanescent wave in the sidepolished region.

Another advantage a side-polished fiber has over a fiber taper is the optical loss. Generally speaking, a total loss on a side-polished fiber is slightly smaller than a fiber taper with a similar length. More importantly, since the cladding of a fiber is removed without touching its core, most of the light will still be traveling inside the core rather than becoming into cladding mode. Less unexpected cladding mode loss makes the final results more accurate and lower in the noise level. Bending loss [14] is another concern. Because a taper cannot be pulled with very large force, it is sometimes bending inside the flow system. Extra loss can be generated because of the bending, and similar as the cladding mode loss, it is difficult to estimate how much loss is related to it. What is more, the bending loss could be changing during a sample measurement. So for each taper it is better to do a ring-down 
time measurement before other tests to get an estimation on total loss. By using a sidepolished fiber the total optical loss is more stable and controllable so further experiments are much easier to operate. Calculations on the evanescent field proportion will be demonstrated and compared in Chapter 4.

The limitations of a side-polished fiber is obvious as well. As can be seen from the schematic in Figure 3.5, since no efforts are done to make thinner core diameter, most of the light is still travelling in the fiber core with only a slight portion of it exposed as evanescent wave. Theoretically, the intensity of evanescent field we will have on a sidepolished fiber is different from what we have from the calculations in Figure 3.1(b) for a taper because its core is untouched so the diameter is larger. Based on Figure 3.4, the evanescent wave can only be detected in a region of a few microns around the core area, and the total intensity is usually less than $1 \%$ of the light intensity. We will further demonstrated the detection results and calculations in Chapter 4 that confirms this estimation. A side-polished fiber sensor contributes a lot in making a low noise level and a stable spectrum output, but its low signal level can be the main restriction on achieving a higher sensitivity of the device.

\subsection{Mirror Reflection System}

After acquiring the detection limit on a side-polished fiber, we find that it impossible to make it work for a detection of $\mathrm{H}_{2} \mathrm{O}$ in a concentration of part per million level, which is 
the level of the saturation $\mathrm{H}_{2} \mathrm{O}$ solution in some common organics such as carbon tetrachloride $\left(\mathrm{CCl}_{4}\right)$ and 1-octyne $\left(\mathrm{C}_{8} \mathrm{H}_{14}\right)$. To improve the efficiency of the light source, we come up with the idea of building up a mirror reflection setup. Instead of the evanescent wave spectroscopy, if a light output from one optical fiber can be directly coupled to another [15], there will be no need to estimate or calculate the evanescent field proportion. By filling the space between the two fibers with liquid samples, it is reasonably to believe that all light in this region is in contact with samples to make the efficiency to be nearly $100 \%$.

The design of the sensing region, shown in Figure 3.6, is made up by holding two fibers together and being vertical to a mirror held in a container. A point on a continuous fiber loop is broken into two halves, and the two exposed fiber ends are held side by side inside a same fiber holder. The holder is designed to hold one fiber so when we put two fibers in, pressure from the holder will squeeze the two fibers to be tightly close to each other. A concave spherical mirror (Gold coating, $\phi 1 / 2 ", 9.5 \mathrm{~mm}$ focal length) is placed under the holder to reflect the light coming out from one fiber to the other. The spherical mirror can help reflect most of the light the same way back to the other fiber because of vertical incidence. Our calculation shows that if the position of the two fiber ends is within a small deviation $(<0.2 \mathrm{~mm}$, compared with a focal length of $9.8 \mathrm{~mm})$, loss on the reflection would be insignificant $(<1 \%)$ compared with the total loss we have on the mirror reflection of 30$40 \%$. 


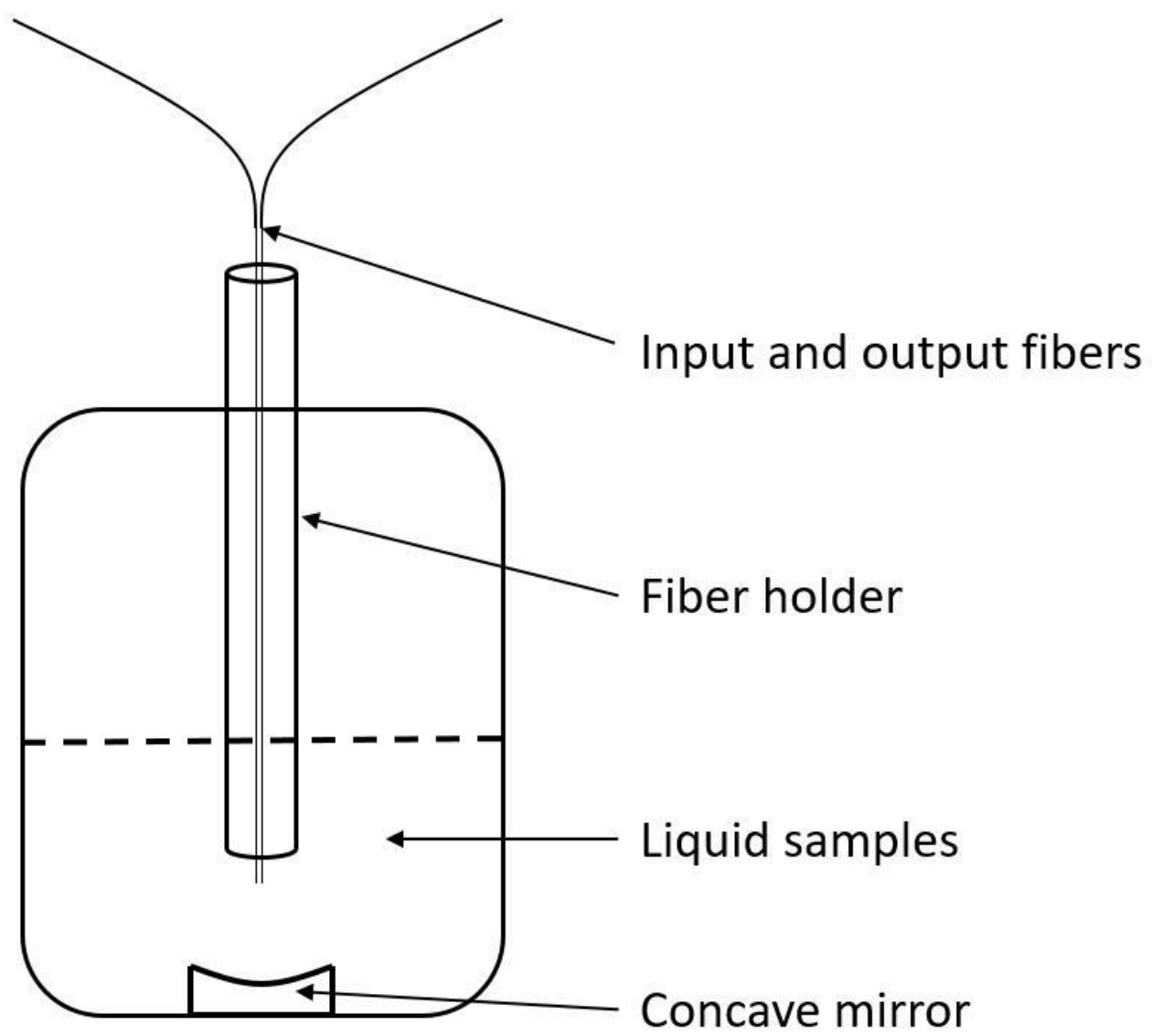

Figure 3.6: Design of the mirror reflection system. Two fibers are held as close as possible and are vertical placed to a mirror. Reflection of light from one fiber to the other helps make improvement on light efficiency.

Assuming that the two fibers are placed close enough to neglect the space between them, it is easy to figure out that the best place to put the fiber ends is at a distance of two focal lengths away from the mirror. Experimental alignment shows the best distance to achieve the highest transmission is at $17.5 \mathrm{~mm}$, and we put it slightly closer to avoid some intensity fluctuation by sacrificing part of the transmission efficiency. With a numerical aperture 
(NA) of the single mode fiber:

$$
N A=n \cdot \sin \theta=\sqrt{n_{\text {core }}^{2}-n_{\text {clad }}^{2}}
$$

Where $\mathrm{n}$ is the refractive index of the surrounding environment. We can have the radius of the light spot on the mirror is:

$$
r=d \cdot \tan \theta=d \cdot N A \cdot \sqrt{\frac{1}{n^{2}-N A^{2}}}
$$

Where $\mathrm{d}$ is the distance from mirror to the fiber ends and $\mathrm{n}$ is the refractive index of the medium. With $\mathrm{NA}=0.14$ for a single mode fiber, in air we can have $\mathrm{r}=0.43 \mathrm{~cm}$. Thus the spot diameter is $0.5 \mathrm{~cm}$ which is smaller than a mirror size $(\phi 1 / 2 ")$. For liquid samples like $\mathrm{H}_{2} \mathrm{O}(\mathrm{n}=1.33)$ and $\mathrm{CCl}_{4}(\mathrm{n}=1.46)$, the diameter could be even smaller.

By implementing this mirror reflection device, we are able to significantly increase the supercontinuum efficiency from less than $1 \%$ in a side-polished fiber to almost $100 \%$ in the sensing region thus improve a signal level by several thousand times. However it goes back with an issue of noise level. Because the design of the sensor become exposed to open air again and the requirement of both longer of time and larger volumes of liquid samples, more fluctuation is observed in the supercontinuum spectra to cause a higher noise level compared to a side-polished fiber sensor. Measurements of organics samples even gives higher noise than air or inorganics samples like $\mathrm{H}_{2} \mathrm{O}$ and $\mathrm{D}_{2} \mathrm{O}$. After some experimental tests we believe most of the higher noise level comes because the organics we test (carbon tetrachloride, acetone, isopropanol, etc.) are all liquids with strong solubility. Unexpected 
contaminates can be easily mixed and dissolved in the samples to cause extra absorbance or noise during an experimental measurement. Even part of the device itself can be unstable in some of those organic environments. The fiber coating, for example, can be corrupted and partly dissolved when immersed in carbon tetrachloride or isopropanol for some time. Efforts are done to attenuate the effect of organics and to lower the noise level, but it is still the most troublesome limitation we have on reaching a more improved signal to noise ratio on this sensing method.

\subsection{Designs for the Flow Systems}

Because of the different in designs of the fiber optical sensors, the flow system we build are also different, especially for the mirror reflection sensor. A closed system is built for the taper and side-polished fiber sensor while the mirror reflection sensor only has an open system because alignments are in need with the free space coupling. The schematics of our flow system designs are shown in Figure 3.7. 

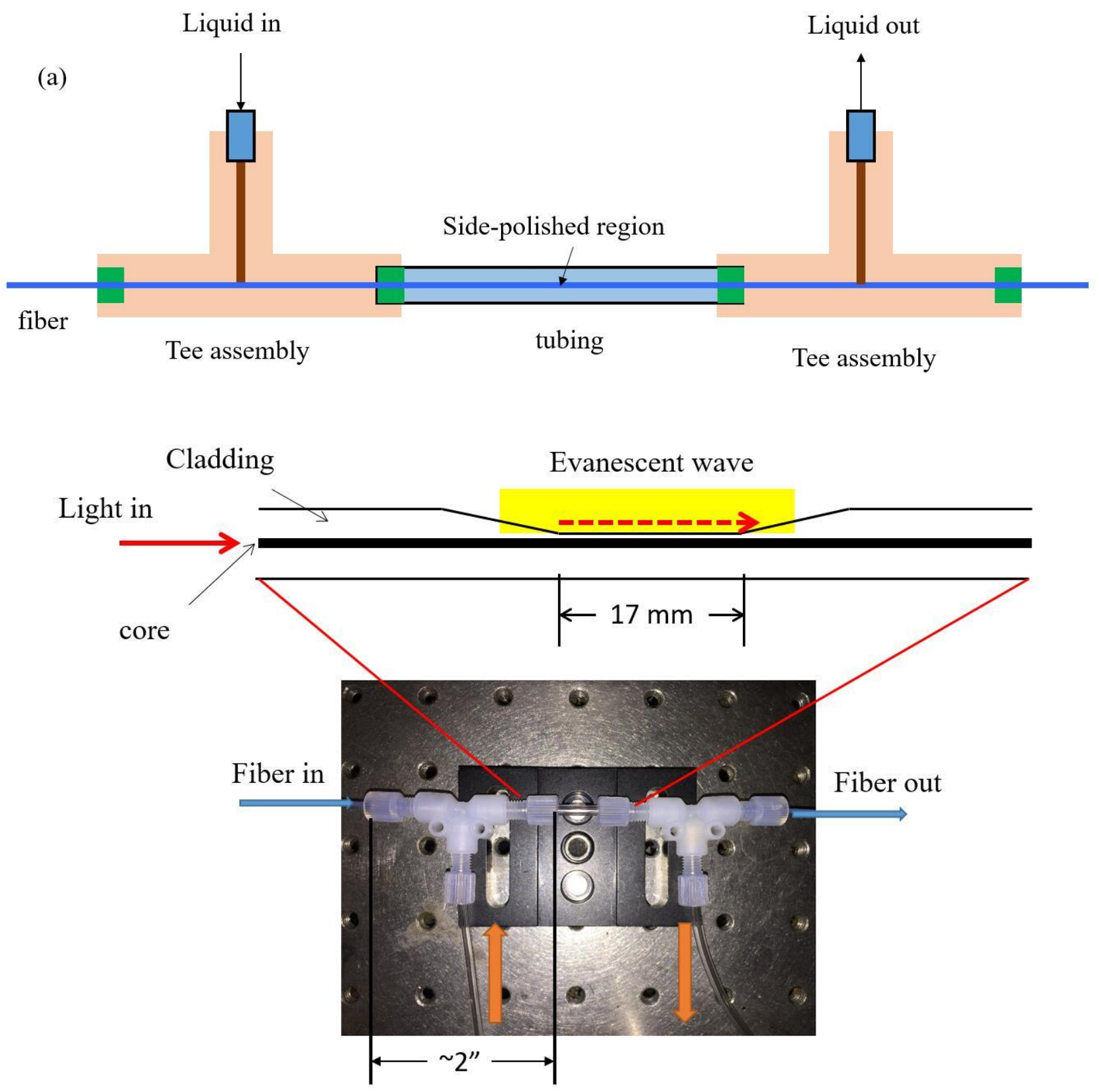

Figure 3.7(a): Schematic of the flow system for a side-polished fiber sensor. Inner diameters of the tee assembly and the tubing are 0.020 " and 0.011 ", respectively. A picture taken for the system on table is attached to show its real size. 


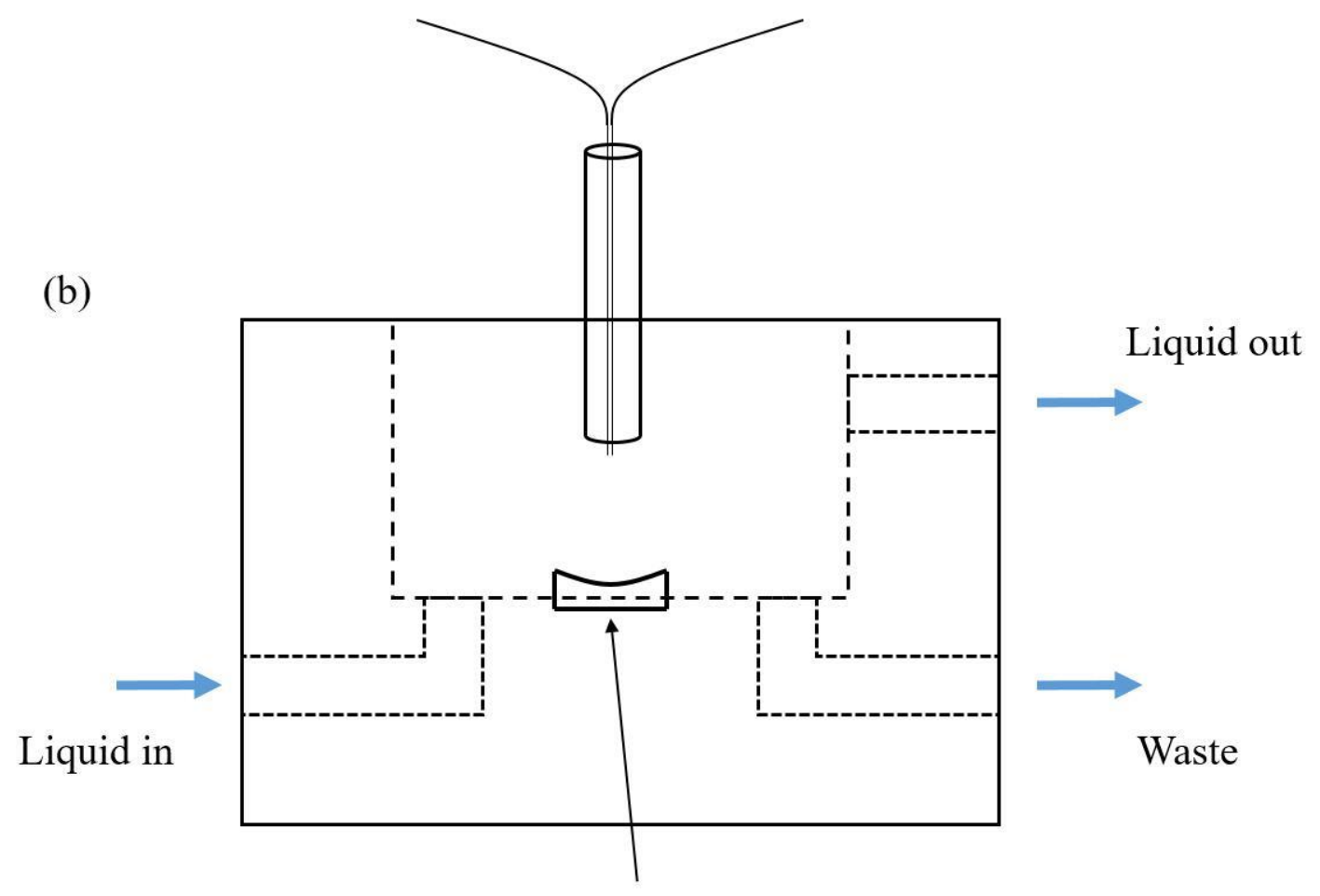

Concave mirror

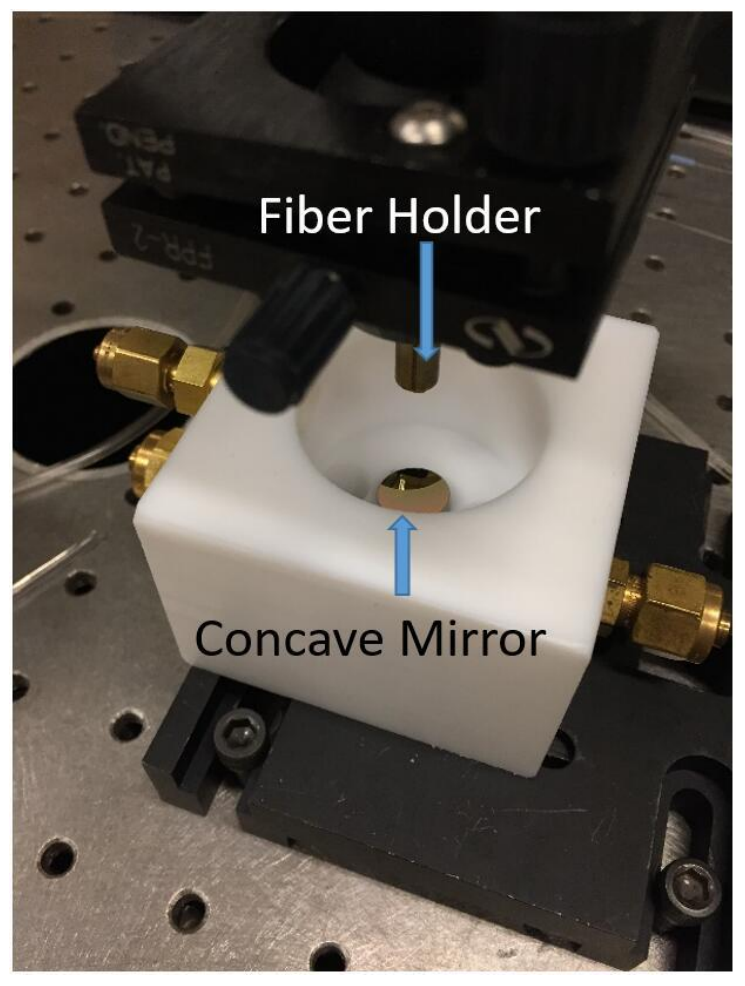

Figure 3.7(b): Flow system designed for the mirror reflection sensor. Inner diameter of the container is $\sim 1$ " and the fibers are placed $\sim 1.7 \mathrm{~cm}$ above the mirror. A real picture is attached for estimation of a sample volume consumed each time. 
Figure 3.7(a) demonstrates the design of the flow systems we have for a side-polished fiber and a taper sensor. It consists of two tee assemblies (Idex Health \& Science, Tee Assembly Tefzel (ETFE), for 1/16" OD, .020" thru hole) and a tubing (Idex Health \& Science, NanoTight Sleeve 1/16" OD x .011" ID x 1.6") held between them. A fiber is held inside the system with its side-polished or tapered region inside the tubing. The pump (Fisher Scientific, Variable-Flow Peristaltic Pumps, Ultralow flow) is connected to the two tee assemblies so it is easy to pump liquid samples in and out and to change samples. Below the schematic we also have a picture of the setup to show the size of this whole sensor. The diameters for the tubing and the through holes in the tee assemblies are very small (Inner diameters of 0.011 " and $0.020 "$, respectively), so each time only a small amount of liquid samples is required to complete a measurement. In average, less than $5 \mathrm{ml}$ of sample is enough to finish a whole experimental procedure including cleaning, rinsing and sensing. For long term tests, the required sample volume is always less than $10 \mathrm{ml}$ per hour. The flow system not only helps to speed up the change of liquid samples, but also contributes to stabilize the supercontinuum spectra. A closed system manages to keep most unexpected influence away and the slow but constant flow of liquids keeps changing liquids inside to make sure the tested samples are new and clean.

Figure 3.7(b) gives the schematic of the flow system we have for the mirror reflection sensor. The mirror is placed inside a container previously made from glass and now switched to Teflon. Two holes are made on either side of the container to connect with 
tubing from the pump. Because of the mirror size and focal length, the container is designed to have 1 " in diameter and 1 " in depth. A shallow $1 / 2$ " hole of $2 \mathrm{~mm}$ deep is made in the bottom of this container so we are able to push the mirror inside to fix it in the bottom without using other adhesives. The fiber holder is above the mirror and both fiber ends are inside to be immersed when the container is filled with liquid samples. A picture of this device is also shown in Figure 3.7(b) to show the size of this system. Based on the size of the container, it takes more than $10 \mathrm{ml}$ of liquids to fill up the whole system. If cleaning and rinsing liquids are included, it could take more than 10 times the volume a side-polished fiber system requires to finish a measurement. The need of larger amount of samples makes the time of a sample test longer because of the restriction on pumping speed. It also makes the use of pump insignificant not only because it takes minutes to pump $\sim 10 \mathrm{ml}$ liquid in, but also a high speed pumping makes unexpected fiber and mirror fluctuations possible during measurements which will lead to bad experimental results.

\subsection{Conclusions}

We have demonstrated three different optical fiber based sensors in this chapter: a fiber taper, a side-polished fiber and a mirror reflection sensor. Each sensor has its own properties that are suitable for this project but also limitations. A taper is a best sensor works with evanescent wave spectroscopy, however our self-made tapers are not robust enough and have an issue to be nonadiabatic. Side-polished fibers are robust and stable enough to give the lowest noise level but their evanescent field intensities cannot match 
with tapers to give a relatively high signal level [16]. The mirror reflection system does not implement the evanescent wave spectroscopy so it has the highest supercontinuum efficiency among all three. It gives fair noise levels but still higher than the side-polished fiber. If the open air system can be improved to closed system and to decrease the requirement on sample volume at the same time, it will make this method the best among all three sensors. Extra noise is observed when organic samples are tested, further improvements on carefully choosing materials to make the device especially in areas where are making contact with liquid samples is required. Finally our designs for the flow system is demonstrated. Generally speaking, a closed system is better than an open one, but for now an open system is in need for the mirror reflection sensor to do optical alignment from time to time. Now most of the sensing is done with the mirror reflection device. In the following chapter we will demonstrate the data and results acquired from these three sensing elements.

\subsection{Reference}

1. Kieu, Khanh Quoc, and Masud Mansuripur. "Biconical fiber taper sensors." IEEE Photonics Technology Letters 18, no. 21 (2006): 2239-2241.

2. Moar, P. N., S. T. Huntington, J. Katsifolis, L. W. Cahill, A. Roberts, and K. A. Nugent. "Fabrication, modeling, and direct evanescent field measurement of tapered optical fiber sensors." Journal of applied Physics 85, no. 7 (1999): 3395-3398. 
3. Ahmad, Mohammad, and Larry L. Hench. "Effect of taper geometries and launch angle on evanescent wave penetration depth in optical fibers." Biosensors and Bioelectronics 20, no. 7 (2005): 1312-1319.

4. Tarsa, Peter B., Paul Rabinowitz, and Kevin K. Lehmann. "Evanescent field absorption in a passive optical fiber resonator using continuous-wave cavity ring-down spectroscopy." Chemical Physics Letters 383, no. 3 (2004): 297-303.

5. Tarsa, Peter B., Aislyn D. Wist, Paul Rabinowitz, and Kevin K. Lehmann. "Single-cell detection by cavity ring-down spectroscopy." Applied Physics Letters 85, no. 19 (2004): $4523-4525$.

6. Ward, J. M., A. Maimaiti, Vu H. Le, and S. Nic Chormaic. "Contributed Review: Optical micro-and nanofiber pulling rig." Review of Scientific Instruments 85, no. 11 (2014): 111501.

7. "Silicon dioxide." https://en.wikipedia.org/wiki/Silicon_dioxide

8. "Adiabatic flame temperature." https://en.wikipedia.org/wiki/Adiabatic_flame_temperature

9. Snyder, Allan W., and John Love. Optical waveguide theory. Springer Science \& Business Media, 2012.

10. Chen, George Y., Ming Ding, Trevor P. Newson, and Gilberto Brambilla. "A review of microfiber and nanofiber based optical sensors." (2013): 32-57.

11. Dong, L., L. Reekie, J. L. Cruz, J. E. Caplen, J. P. De Sandro, and D. N. Payne. "Optical fibers with depressed claddings for suppression of coupling into cladding modes in 
fiber Bragg gratings." IEEE Photonics Technology Letters 9, no. 1 (1997): 64-66.

12. Tseng, Shiao-Min, and Chin-Lin Chen. "Side-polished fibers." Applied optics 31, no. 18 (1992): 3438-3447.

13. Gaston, Ainhoa, Ibon Lozano, Fátima Perez, Fernando Auza, and Joaquín Sevilla. "Evanescent wave optical-fiber sensing (temperature, relative humidity, and $\mathrm{pH}$ sensors)." IEEE Sensors Journal 3, no. 6 (2003): 806-811.

14. Bobb, Lloyd C., P. M. Shankar, and Howard D. Krumboltz. "Bending effects in biconically tapered single-mode fibers." Journal of lightwave technology 8, no. 7 (1990): 1084-1090.

15. Marcuse, Dietrich, and Julian Stone. "Coupling efficiency of front surface and multilayer mirrors as fiber-end reflectors." Journal of Lightwave Technology 4, no. 4 (1986): 377-381.

16. Henry, W. "Evanescent field devices: a comparison between tapered optical fibres and polished or D-fibres." Optical and quantum electronics 26, no. 3 (1994): S261-S272. 


\section{Chapter 4 Liquid Phase Sensing of $\mathrm{H}_{2} \mathrm{O}$ in $\mathrm{D}_{2} \mathrm{O}$ and Organics}

$\mathrm{H}_{2} \mathrm{O}$ is an elementary compound that has long been a wide interest for a long time. Water vapor [1], liquid water [2] and solid ice [3], pure or mixed with other compounds, all appear in many works ever since several decades ago. [4] Water detection provides an attractive way to demonstrate and examine our newly built device. There is an $\mathrm{O}-\mathrm{H}$ bond stretching vibration overtone absorption peak in the region around 1450nm for pure liquid $\mathrm{H}_{2} \mathrm{O}$ [5] which matches with the wavelength coverage of the supercontinuum (600nm to $1600 \mathrm{~nm})$ and spectrometer (900nm to $1700 \mathrm{~nm}$ ). The position of this absorption peak is observed to move to lower wavelength when $\mathrm{H}_{2} \mathrm{O}$ is made in liquid mixtures. The reference spectrum is chosen to be $\mathrm{D}_{2} \mathrm{O}$, which has similar behavior as $\mathrm{H}_{2} \mathrm{O}$ but does not show the high absorption feature as $\mathrm{H}_{2} \mathrm{O}$ does around $1450 \mathrm{~nm}$ because the O-D bond stretching overtone is less in intensity and shifted in peak position. In this project, $\mathrm{H}_{2} \mathrm{O}$ spectra with different concentrations in $\mathrm{D}_{2} \mathrm{O}$ was measured with the three different sensors introduced above to show how each sensor works in the device. The samples are further expand to $\mathrm{H}_{2} \mathrm{O}$ spectra in organics like carbon tetrachloride $\left(\mathrm{CCl}_{4}\right)$ with the mirror reflection sensor since it is made to improve the efficiency of the light source in the fiber-loop. In this chapter we will demonstrate the results we have for different samples with the three sensors and some discussions are made as well. 


\section{1 $\mathrm{H}_{2} \mathrm{O}$ in $\mathrm{D}_{2} \mathrm{O}$ with Fiber Tapers}

The reason we start with $\mathrm{H}_{2} \mathrm{O}$ and $\mathrm{D}_{2} \mathrm{O}$ sample pair is they are miscible with any concentration mixtures. The reference spectrum is always the pure $\mathrm{D}_{2} \mathrm{O}$, and $\mathrm{H}_{2} \mathrm{O}$ is dissolved in with different amount to make several samples with different $\mathrm{H}_{2} \mathrm{O}$ percentage

for tests of the device. Each time the spectra are scanned under 20ms integration time and averaged 100 times. A ratio of the absorption spectrum of the sample with the reference sample is made and then based on Beer's Law [6], an absorbance can be acquired:

$$
A=-\log \left(\frac{I}{I_{0}}\right)=\sigma \cdot N \cdot L=\alpha \cdot L
$$

Where $\mathrm{A}$ is the total absorbance, $\mathrm{I}$ and $\mathrm{I}_{0}$ are the light intensities of sample and reference respectively, $\sigma$ is the molecular absorption cross section for the sample, $\mathrm{N}$ is the number density, $\mathrm{L}$ is the absorption optical path length and $\alpha$ is the absorption coefficient for the sample. Samples with known concentrations are tested first so our calculation are focused on getting an effective optical path for each sensor and estimating a light efficiency for it.

For fiber tapers, it is difficult to control the optical loss in the fabrication process, so we directly connect the fiber with a power meter on the pulling device and keep a laser running through it during the whole pulling process. The tapers with low optical loss are thus selected to work as sensors. However, this power monitor only gives a rough understanding of a total loss on the sensor but does not give any information on the signal level we can be expecting because it is hard to tell the waist diameter of a taper. As stated before, tapers with different waist diameters will have significant different fraction of intensity in the 
evanescent field that interacts with the samples $[7,8]$, which makes the signal level and signal to noise ratio differs from one taper to another. Furthermore, it is difficult to take SEM images of a taper, increasingly as the tapers get narrower. A part from a taper sample has to be removed and put into the chamber to do SEM imaging, which means it can no longer be used as a sensor. Our SEM images are taken only from used or broken tapers.

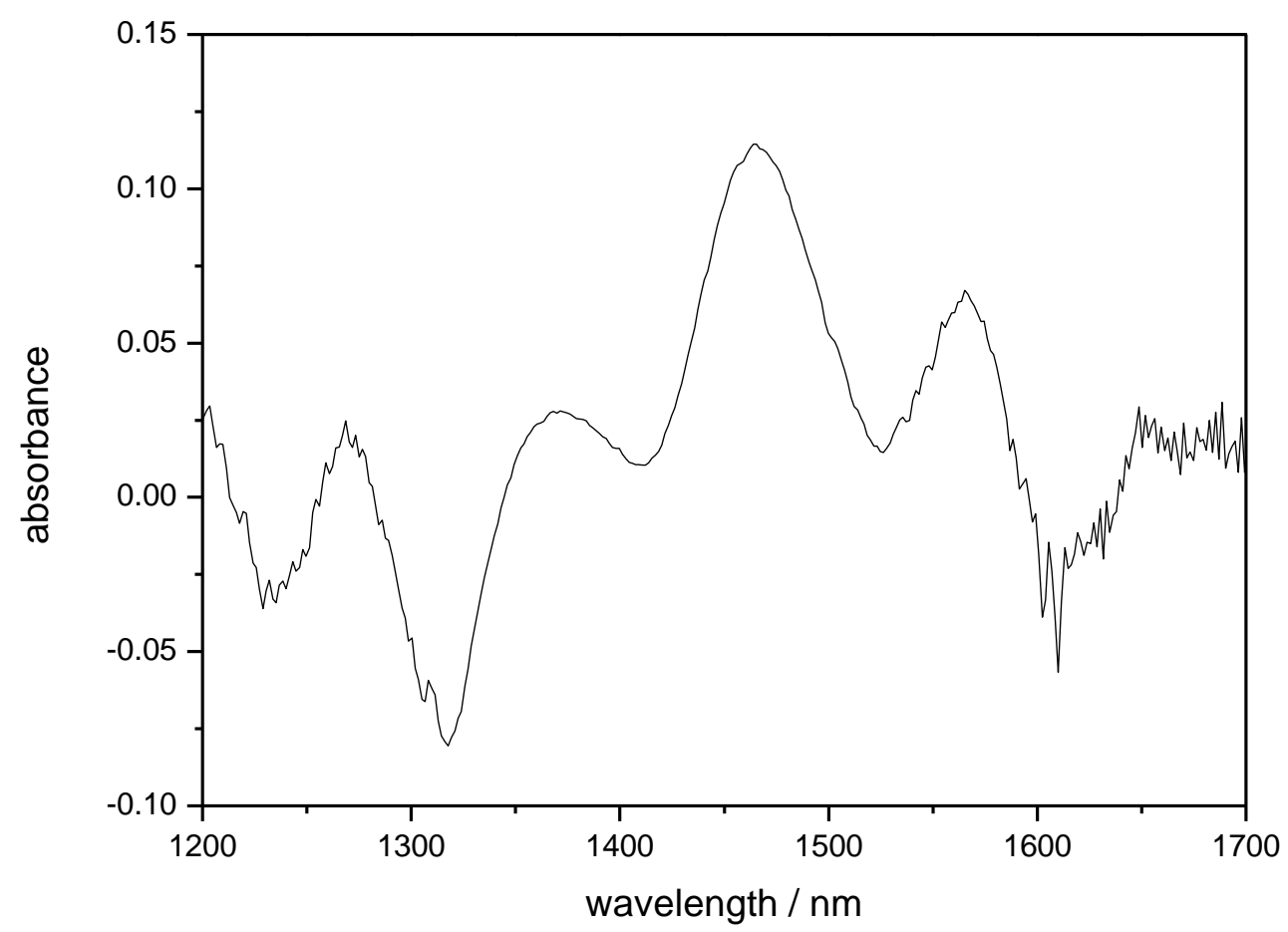

Figure 4.1: Absorbance of pure $\mathrm{H}_{2} \mathrm{O}$ measured by a fiber taper sensor.

Figure 4.1 shows an absorption spectrum we take from one taper sensor. The sample here is pure liquid $\mathrm{H}_{2} \mathrm{O}$. The measurements are done for several times for the taper and the peak observed near $1450 \mathrm{~nm}$ is at the same position as other sensors. However, there are other peaks that we are not able to explain clearly. Another absorption peak next to the $1450 \mathrm{~nm}$ 
absorption appears on both side-polished fiber and mirror reflection device near 1520nm, but only on the samples of $\mathrm{H}_{2} \mathrm{O}$ solutions in $\mathrm{D}_{2} \mathrm{O}$. Pure $\mathrm{H}_{2} \mathrm{O}$ sample gives only one absorption feature so the second peak could be due to the formation of HDO in the solutions. However, this extra peak in we find on a taper sensor could be due to the sensor itself. The absorptions in $1200 \mathrm{~nm}$ to $1300 \mathrm{~nm}$ are peaks not appearing with the other two sensors. Possible reasons are the tapers not being adiabatic for the light propagating in. The interference of light beams in core mode and cladding mode [9] may cause some of these apparent peaks, which can also make the real absorbance we have on $\mathrm{H}_{2} \mathrm{O}$ sample to be not accurate.

Another issue we have with the tapers is they do not give a signal level as high as expected. The absorbance observed at 1450nm in Figure 4.1 is less than 10 times higher of what is got from the side-polished fiber sensor, which only gives an evanescent field intensity of $<0.02 \%$ of the total supercontinuum intensity travelling inside the loop. (Calculations will be demonstrated in the part of results for side-polished fibers.) This means the evanescent field intensity for a taper is also far below a proportion of $1 \%$ of the total supercontinuum light source. Mainly due to the waist diameter not being thin enough, the evanescent field is not comparable with the strength it can be at a waist of $20 \mu \mathrm{m}$ and below. In terms of this, the tapers cannot match with the side-polished fibers we use in this project since they give a signal level only a few times higher but are more fragile and with unstable noise level. Furthermore, the evanescent field intensity can be significantly different with 
different fibers. Sometimes a $1-2 \mu \mathrm{m}$ reduction in a taper waist can double the evanescent field intensity, especially when the waist is already thin enough because the incident angle can be very close to the critical angle (Seen from Figure 3.4). The pulling device has to be further redesigned with more sensitive fiber alignment and a camera to observe the pulling process which requires more tests with more careful operations, so for now we believe a side-polished fiber is a better option. Improvements have also to be done on controlling the hydrogen flame size and temperature. Enclosing the pulling setup in a box and using a more precise flow meter for $\mathrm{H}_{2}$ and $\mathrm{O}_{2}$ gases were implemented, however there are still room for improvement. Random air current brought by igniting the flame and even small particles attached on fiber or torch can make the flame not as stable as expected. More works on fiber pulling is required, a fully manufactured commercial taper with predetermined evanescent field intensity and optical loss is a better option before the whole improvement is finished and tested.

\section{2 $\mathrm{H}_{2} \mathrm{O}$ solutions in $\mathrm{D}_{2} \mathrm{O}$ with Side-polished Fiber Sensors}

Compared with a fiber taper, a side-polished fiber is obviously more stable and robust. A clear improvement we notice is that when a taper is replaced by a side-polished fiber inside the flow system made by the two tee assemblies, no extra optical loss is recorded. We believe this is because it is easy to pull a side-polished fiber straight inside the flow system while usually we do not dare to put extra force on a taper which will cause some bending loss [10], and if a taper is in touch with the inner wall of the tubing, more loss could be 
introduced. As we stated in Chapter 2, several wavelengths are separated by interference filters to record their ring-down signal and do an exponential decay fit for their ring-down time. With the ring-down time we determine the optical loss in each wavelength regions and necessary adjustments on the effective round trip times are made when we do final calculations.

With the $\mathrm{O}-\mathrm{H}$ bond absorbance centered at $1450 \mathrm{~nm}$ for pure $\mathrm{H}_{2} \mathrm{O}$ and between $1400 \mathrm{~nm}$ and 1450nm for $\mathrm{H}_{2} \mathrm{O}$ at different concentrations in different solutions, our focus is the supercontinuum in the wavelength range of 1300 to $1500 \mathrm{~nm}$. For a side-polished fiber, the same ring-down time fit is not only done with an empty resonant cavity (pump off with only air in the flow system), but it is also done in the environment of pure $\mathrm{H}_{2} \mathrm{O}$ and $\mathrm{D}_{2} \mathrm{O}$ surroundings with the flow system on. A same procedure is done to record the ring-down signal and then fit the decay to obtain a ring-down time for each different wavelength band centered at $1300 \mathrm{~nm}, 1400 \mathrm{~nm}, 1500 \mathrm{~nm}$ and $1550 \mathrm{~nm}$. The comparison of ring-down times for a side-polished fiber in pure $\mathrm{H}_{2} \mathrm{O}$ and $\mathrm{D}_{2} \mathrm{O}$ environment have already been demonstrated in Figure 2.3. The bandwidth (FWHM) of the interference filters is $2 \mathrm{~nm}$, which is almost the same as the resolution of the spectrometer $(1.56 \mathrm{~nm})$. For the wavelengths of $1400 \mathrm{~nm}$ and $1500 \mathrm{~nm}$, the difference in ring-down times between a $\mathrm{H}_{2} \mathrm{O}$ and a $\mathrm{D}_{2} \mathrm{O}$ environment is small because $\mathrm{H}_{2} \mathrm{O}$ absorbance on a side-polished fiber is small of only $\sim 0.02$ for pure $\mathrm{H}_{2} \mathrm{O}$. The final round trip loss results can be found in Table 4.1 listed below after the calculations based on the absorbance. 
The steps to take a sample spectrum start with the background or dark spectrum of the spectrometer itself. Then a reference spectrum is taken by subtracting the background value from a spectrum of a pure $\mathrm{D}_{2} \mathrm{O}$ sample. A sample spectrum is taken afterwards on pure $\mathrm{H}_{2} \mathrm{O}$ or mixtures of $\mathrm{H}_{2} \mathrm{O}$ and $\mathrm{D}_{2} \mathrm{O}$. The mixtures are made by making combinations of $\mathrm{H}_{2} \mathrm{O}$ and $\mathrm{D}_{2} \mathrm{O}$ in different volumes. For example, a $10 \% \mathrm{H}_{2} \mathrm{O}$ solution in $\mathrm{D}_{2} \mathrm{O}$ means $10 \mathrm{ml}$ of deionized $\mathrm{H}_{2} \mathrm{O}$ is taken and diluted to $100 \mathrm{ml}$ with $\mathrm{D}_{2} \mathrm{O}$ in a $100 \mathrm{ml}$ volumetric flask. A ratio of the sample and the reference spectra is computed. The logarithm of the ratio is the calculated absorbance for a sample at the specific wavelength. Finally by the calculated absorbance, we are able to know the working abilities of the sensing element in use.

Figure 4.2 gives the data taken from side-polished fiber sensors. In both spectra, a larger integration time is set on the spectrometer so the region of $1200 \mathrm{~nm}$ to $1300 \mathrm{~nm}$ is saturated on the spectrometer to make a reasonably higher intensity level in $1300 \mathrm{~nm}$ to $1500 \mathrm{~nm}$ to make full use of the spectral coverage in the region where the absorption peak lies in. Figure 4.2(a) is a spectrum on pure $\mathrm{H}_{2} \mathrm{O}$ sample taken against the reference of $\mathrm{D}_{2} \mathrm{O}$. An obvious signal level is obtained for the absorption and the peak position corresponds well with some previous work on pure $\mathrm{H}_{2} \mathrm{O}$ [5]. Based on the figure, the absolute value of this absorbance is low to be only $<0.020$. Comparing with a bulk absorption coefficient of $26 \mathrm{~cm}^{-1}$ [4], it is easy to figure out that only a slight portion of the supercontinuum is working as the evanescent field outside the side-polished fiber. Here we will briefly demonstrate the process of calculations of the evanescent field intensity proportion. 
(a)

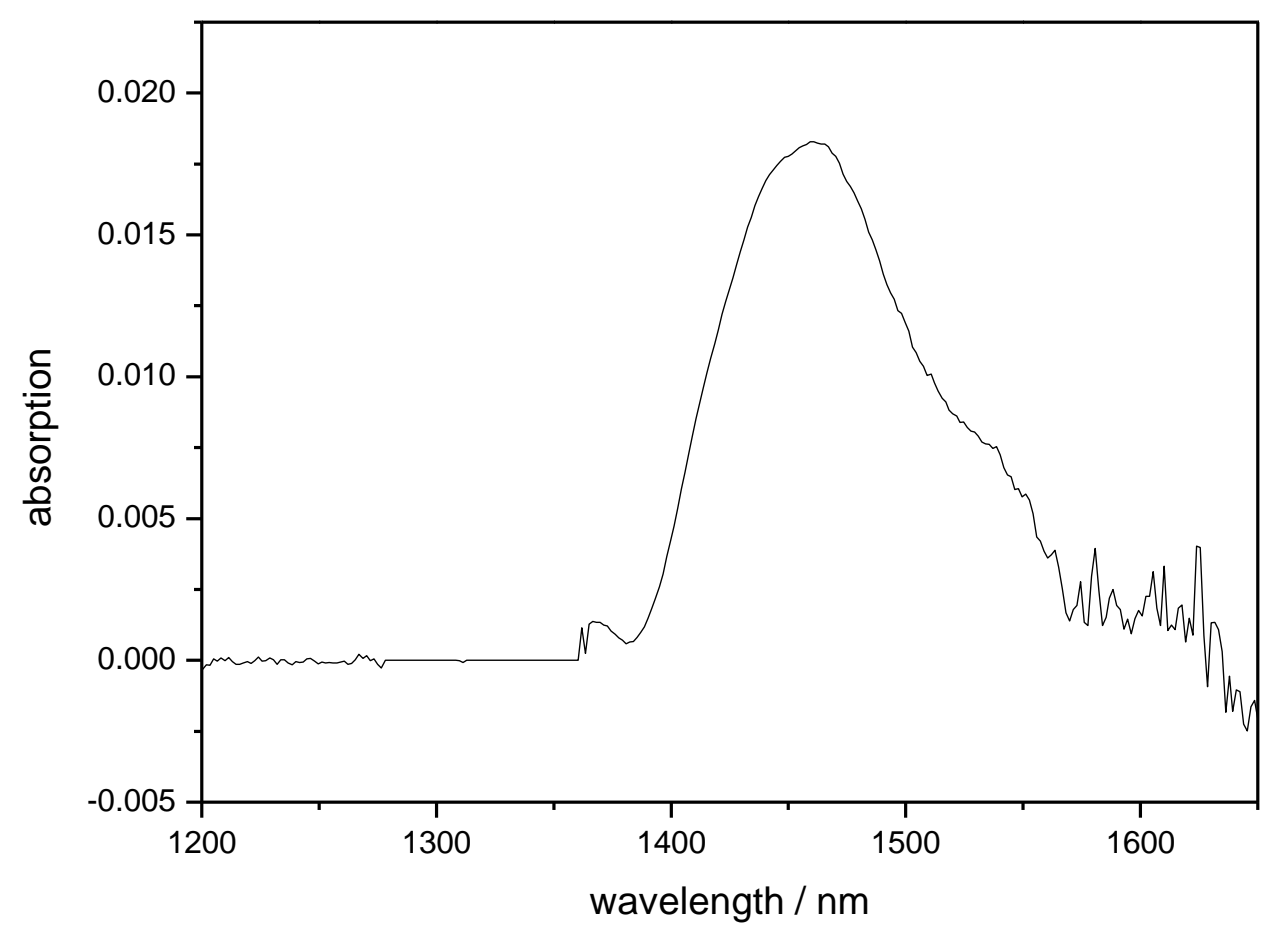

(b)

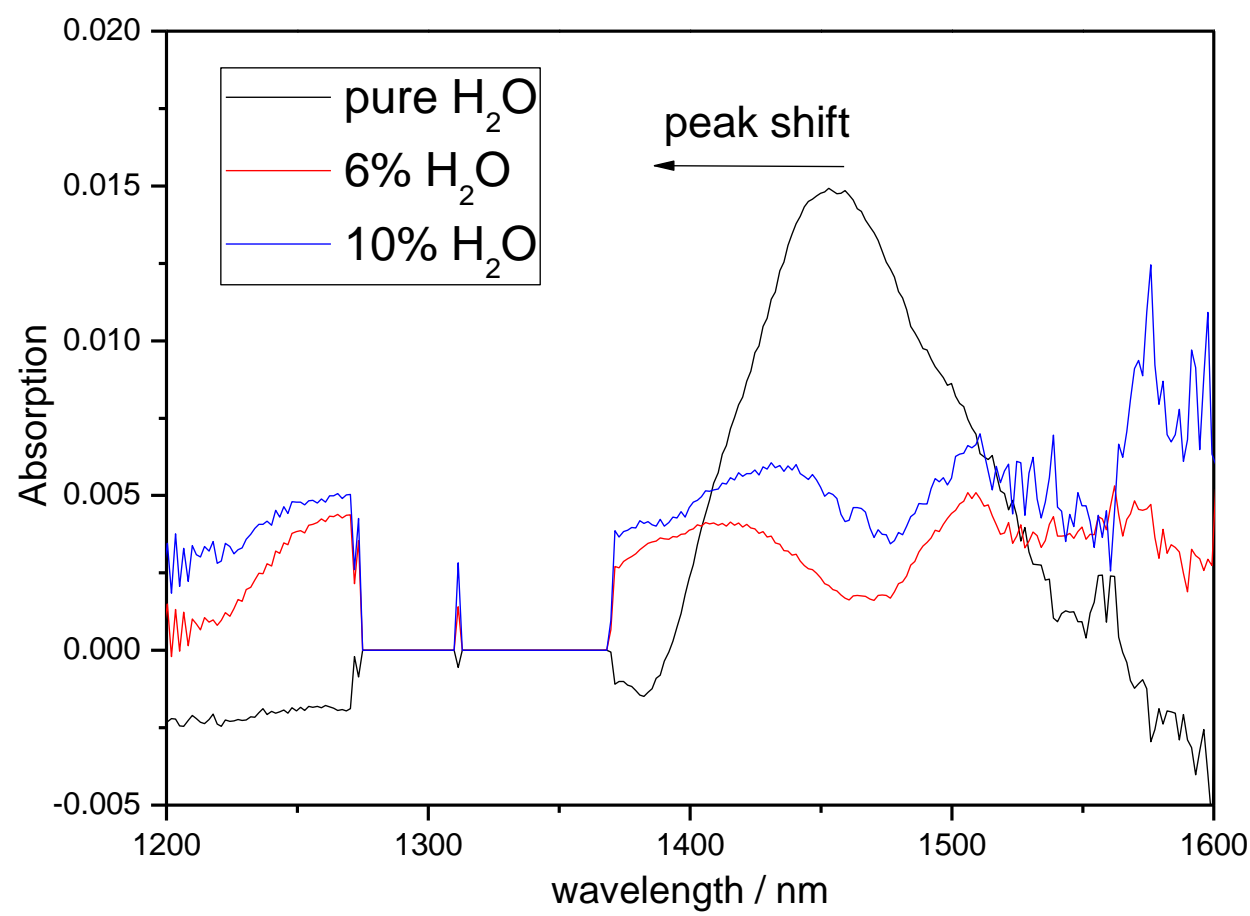

Figure 4.2: Absorbance of $\mathrm{H}_{2} \mathrm{O}$ samples measured by a side-polished fiber sensor. (a) pure $\mathrm{H}_{2} \mathrm{O}$ sample; (b) comparison of pure $\mathrm{H}_{2} \mathrm{O}$ sample with $10 \%$ and $6 \% \mathrm{H}_{2} \mathrm{O}$ samples in $\mathrm{D}_{2} \mathrm{O}$. Pure $\mathrm{D}_{2} \mathrm{O}$ is the reference in both measurements. 
Assuming the total absorption coefficient is $\alpha$ for $\mathrm{H}_{2} \mathrm{O}$, based on the absorbance we have in Figure 4.2(a), we can have an effective absorption length to be:

$$
L_{\mathrm{eff}}=\frac{\mathrm{A}}{\alpha}
$$

A total absorption length can be represented as:

$$
L=x \cdot l
$$

Where $x$ is the round trip times and $l$ is the total length of the side-polished region. So an evanescent field proportion can be taken from the effective length to be:

$$
\eta_{\text {eva }}=\frac{L_{e f f}}{L}
$$

With the absorbance measured in Figure 4.2 and the ring-down time from Figure 2.3, the round trip times can be got from $1400 \mathrm{~nm}$ and $1500 \mathrm{~nm}$. If we assume the round trip time for $1450 \mathrm{~nm}$ is the mid-point between $1400 \mathrm{~nm}$ and $1500 \mathrm{~nm}$, a final number can be calculated and the results are listed in Table 4.1.

Table 4.1: Results for $\mathrm{H}_{2} \mathrm{O} / \mathrm{D}_{2} \mathrm{O}$ measurements done with a side-polished fiber

\begin{tabular}{|c|c|c|c|}
\hline & Ring-down time & Round trip loss & Round trip times \\
\hline $1400 \mathrm{~nm}$ & $6.24 \mu \mathrm{s}$ & $27 \%$ & 2.42 \\
\hline $1500 \mathrm{~nm}$ & $8.11 \mu \mathrm{s}$ & $21 \%$ & 3.14 \\
\hline Average & $7.18 \mu \mathrm{s}$ & $24 \%$ & 2.78 \\
\hline $\mathrm{H}_{2} \mathrm{O}$ absorption coefficient $\alpha=26 \mathrm{~cm}^{-1}$ & $L_{\mathrm{eff}}=\frac{\mathrm{A}}{\alpha}=7.04 \times 10^{-4} \mathrm{~cm}$ \\
\hline \multicolumn{2}{|c|}{$L=x \cdot l=47.26 \mathrm{~mm}$} \\
\hline \multicolumn{2}{|c|}{ Side-polished length: $l=17 \mathrm{~mm}_{\text {eva }}=\frac{L_{\text {eff }}}{L}=0.00015=0.015 \%$} \\
\hline
\end{tabular}


As listed in Table 4.1, after the numbers from the figures are implanted in the equations, a final number of a percentage of less than $0.02 \%$ is found for the evanescent field compared with the total intensity of supercontinuum. Different from a fiber taper, for a side-polished fiber, it is the fiber core radius that defines how large the evanescent field intensity is. Based on the work done by Tseng et al. [11], a fiber with core diameter of $9.8 \mu \mathrm{m}$ and cladding of 1 to $2 \mu \mathrm{m}$, the attenuation coefficient should be lower than $1 \times 10^{-3}$, which agrees well with the percentage from this work.

Based on the absorption peak we have on pure $\mathrm{H}_{2} \mathrm{O}$ sample in Figure 4.1(a), a signal to noise level of $>100$ is obtained. Figure 4.2(b) is a comparison of pure $\mathrm{H}_{2} \mathrm{O}$ sample with some mixed samples of $\mathrm{H}_{2} \mathrm{O}$ and $\mathrm{D}_{2} \mathrm{O}$. Concentrations of $10 \%$ and $6 \% \mathrm{H}_{2} \mathrm{O}$ in $\mathrm{D}_{2} \mathrm{O}$ are made, and the reference is also pure $\mathrm{D}_{2} \mathrm{O}$. There is a clear redshift on the peak position of the total absorbance and the less $\mathrm{H}_{2} \mathrm{O}$, the lower wavelength the peak is. This redshift is reported in some other works as well [12], and the most possible reasons can be the formation of $\mathrm{HDO}$ when $\mathrm{H}_{2} \mathrm{O}$ and $\mathrm{D}_{2} \mathrm{O}$ is mixed. In the solution there is an equilibrium of:

$$
\mathrm{H}_{2} \mathrm{O}+\mathrm{D}_{2} \mathrm{O} \rightleftharpoons 2 \mathrm{HDO}
$$

This equilibrium has an equilibrium constant of $\mathrm{K}=3.76$ at $25^{\circ} \mathrm{C}$ [13], which means roughly there will be $18 \% \mathrm{HDO}$ and only $1 \% \mathrm{H}_{2} \mathrm{O}$ existing in a $10 \% \mathrm{H}_{2} \mathrm{O}$ solution in $\mathrm{D}_{2} \mathrm{O}$ when the equilibrium is reached. The absorption feature for different species of $\mathrm{H}_{2} \mathrm{O}, \mathrm{D}_{2} \mathrm{O}$ and $\mathrm{HDO}$ are slightly different due to different density and length of hydrogen bonds. [14] The noise level on the mixed samples also becomes higher than pure $\mathrm{H}_{2} \mathrm{O}$, but with the $6 \%$ sample a 
signal to noise of $\sim 5$ can still be reached which makes it possible to use the side-polished fiber in $\mathrm{H}_{2} \mathrm{O}$ detections of a concentration lower than $5 \%$.

The comparison between a side-polished fiber and a fiber taper is focused on the signal levels they can provide. Even though our self-made fiber taper fails to prove its advance in providing more signal intensity (i.e. evanescent field intensity in this work), many other works $[15,16]$ and some of our previous work [17] all show what a well fabricated fiber taper can achieve in liquid phase sensing. On the other hand, a side-polished fiber is more robust and easier to handle, which makes it better to work as a portable sensor.

\subsection{Results for Mirror Reflection Device}

The main reason we change from a side-polished fiber to a mirror reflection is due to the low evanescent field intensity on the side-polished fibers. Since the detection limit of a side-polished fiber only makes to several percent of $\mathrm{H}_{2} \mathrm{O}$ concentration, which makes it unable to do measurement with the amount of $\mathrm{H}_{2} \mathrm{O}$ in organics immiscible with $\mathrm{H}_{2} \mathrm{O}$. The switching of sensors significantly improves the signal by thousands times larger than the other two sensors. As in the calculations we show in Table 4.1 for the side-polished fibers, assuming the final percentage increases from $0.015 \%$ to $100 \%$ and to derive it back, a total absorbance of $\sim 120$ can be achieved. Although some parameters like round trip loss would be higher here due to a higher absorption and the reflection coupling efficiency, the final signal level is still improved by a factor of $10^{3}$. 
(a)

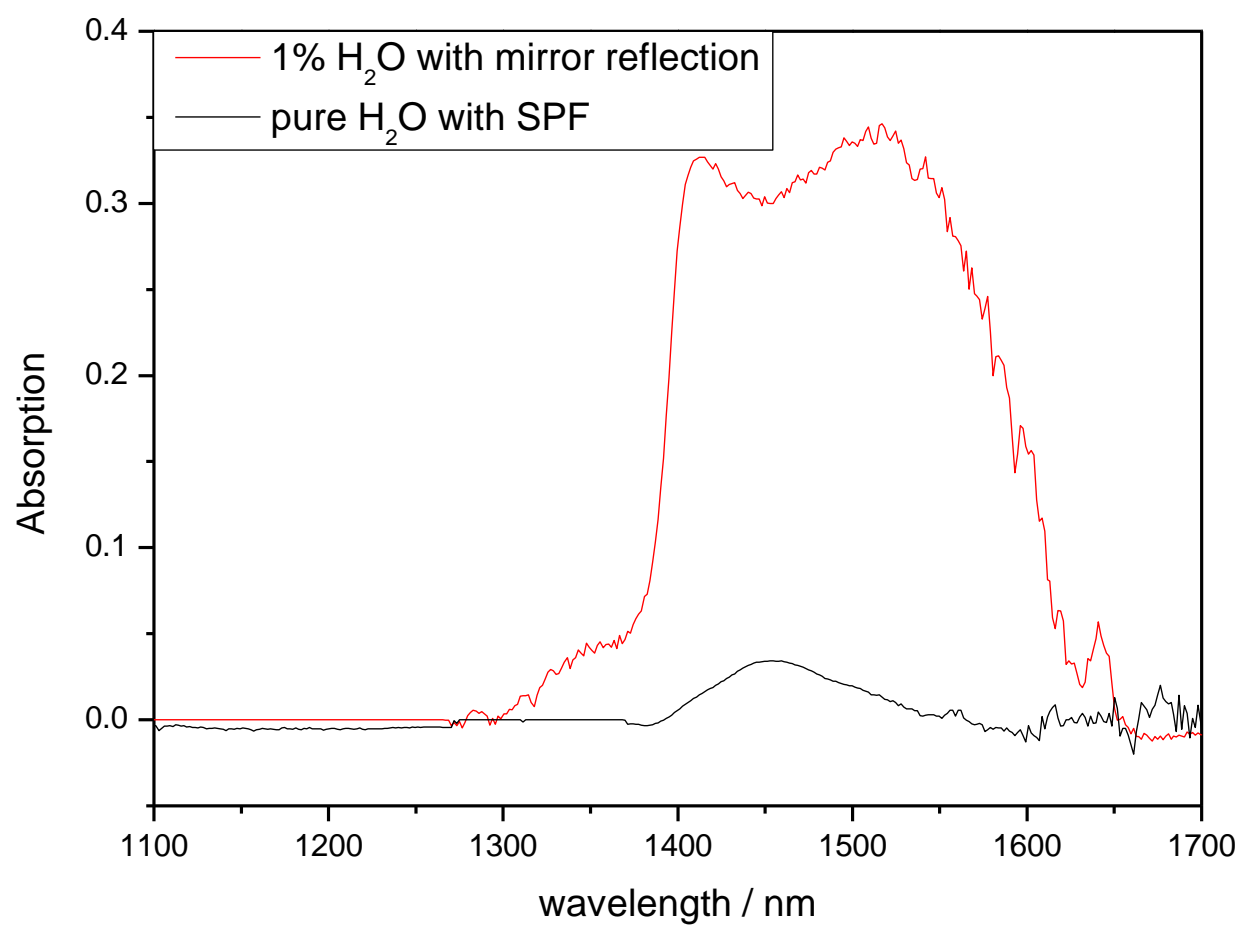

(b)

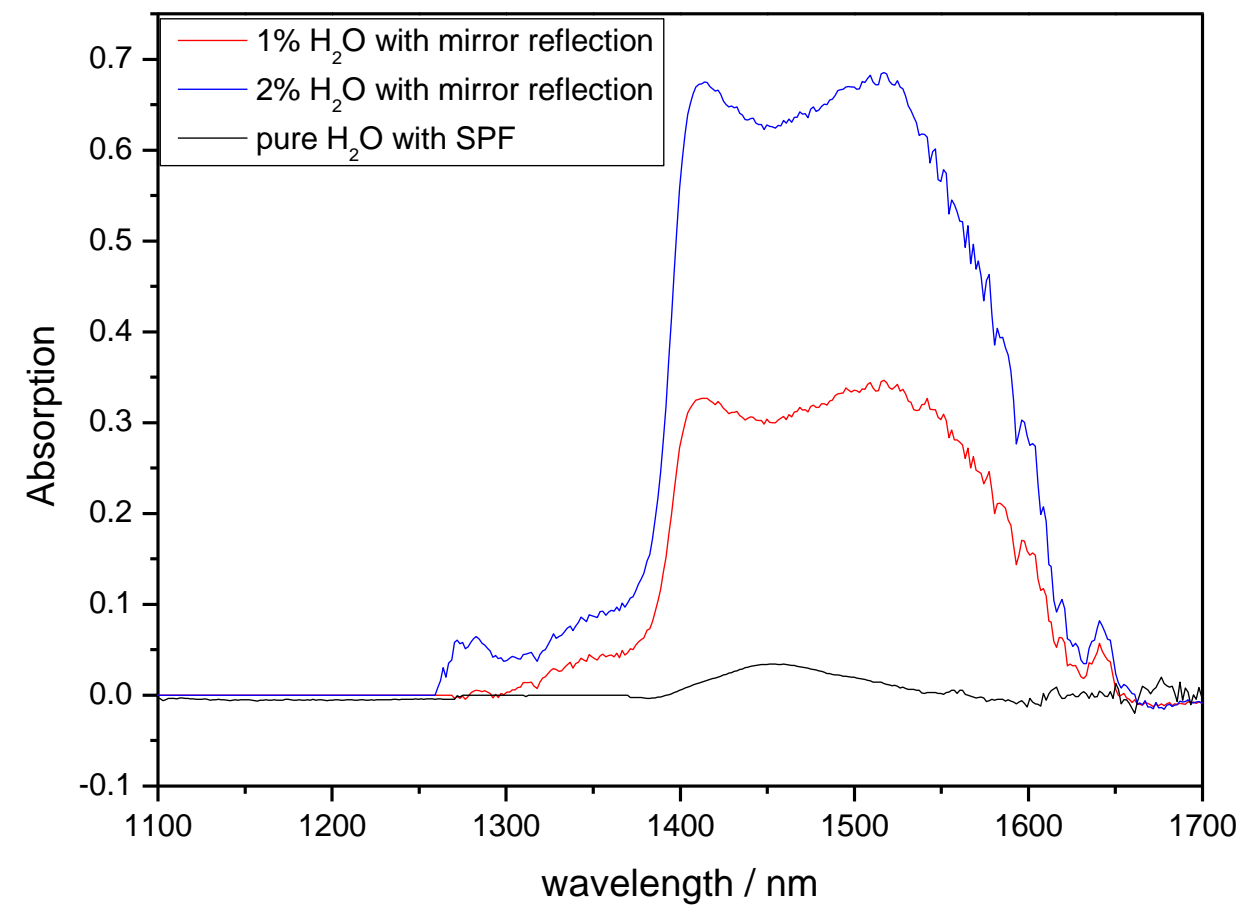

Figure 4.3: Absorbance of $\mathrm{H}_{2} \mathrm{O}$ samples measured by a mirror reflection sensor. (a) $1 \%$ $\mathrm{H}_{2} \mathrm{O}$ solution in $\mathrm{D}_{2} \mathrm{O}$; (b) $1 \%$ and $2 \% \mathrm{H}_{2} \mathrm{O}$ solution in $\mathrm{D}_{2} \mathrm{O}$. Comparison with pure $\mathrm{H}_{2} \mathrm{O}$ sample from the side-polished fiber sensor is made in both figures. 
The test for the mirror reflection system also starts with pure $\mathrm{D}_{2} \mathrm{O}$ as a reference and samples of $\mathrm{H}_{2} \mathrm{O}$ in $\mathrm{D}_{2} \mathrm{O}$ solutions are taken. Because the estimation of signal increase is high, only $1 \%$ and $2 \% \mathrm{H}_{2} \mathrm{O}$ solutions are made as samples to be tested. Figure 4.3 gives the results we have on these liquid samples. The absorbance are acquired the same way we do with $\mathrm{H}_{2} \mathrm{O}$ samples with a side-polished fiber and two spectra from different sensors are further compared with their signal and noise.

Figure 4.3(a) is the absorbance spectrum of the $1 \% \mathrm{H}_{2} \mathrm{O}$ solution. Position of the absorption peak has further redshifted from 1450nm to less than 1410nm [18] and with an even lower concentration than the ones made with side-polished fiber measurements. The black curve is the pure $\mathrm{H}_{2} \mathrm{O}$ spectrum from side-polished fiber results. It is clear the absorbance increases from $\sim 0.020$ to almost 0.35 even with a 100-time concentration decrease. So it is reasonable to estimate when a same sample is used in both the side-polished fiber and the mirror reflection, a more than 1000 times signal improvement can be expected. Another point we notices is that since the absorbance is high at most of the region between 1400 and $1500 \mathrm{~nm}$, it makes the implement of fiber-loop not as necessary as it is with a sidepolished fiber sensor. Because the high absorption of $\mathrm{H}_{2} \mathrm{O}$ in this region, most absorption happens at the first round trip. Light intensity in the second round trip is estimate to be less than $40 \%$ of the first one which means an effective round trip number is only slightly more than 1 . If we take the extra loss on the reflection coupling into concern, this number could be even smaller. Because a little tradeoff is made on the coupling efficiency to make the 
spectrum more stable and with less fluctuation, the free space coupling loss could be as high as $40 \%$ in the final measurements. Based on the signal and noise level from the figures, a detection limit of a concentration of $0.02 \% \mathrm{H}_{2} \mathrm{O}$ can be reached on the mirror reflection sensor.

Another absorption feature overlapped with the $1410 \mathrm{~nm} \mathrm{H}_{2} \mathrm{O}$ peak is observed at $1520 \mathrm{~nm}$. Similar peaks are observed in the spectra of $6 \%$ and $10 \% \mathrm{H}_{2} \mathrm{O}$ as well in the tests done on side-polished fibers shown in Figure 4.2(b) but a little buried in the noise. Similar feature does not appear on the pure sample or $\mathrm{H}_{2} \mathrm{O}$-organic samples, so this is also believed to be due to the formation of HDO inside the mixed samples.

In Figure 4.3(b) a spectrum taken from $2 \% \mathrm{H}_{2} \mathrm{O}$ solution is added to be compared with the $1 \%$ sample and the pure sample from side-polished fiber test. The absorbance doubles at $1410 \mathrm{~nm}$ to $\sim 0.7$ which indicates that almost $80 \%$ of the supercontinuum intensity is absorbed within its first round trip in the loop. For an absorption as high as this, it makes no difference if a fiber-loop is in use or not. The double pass from a one-time mirror reflection has already made the same absorption level on the spectrometer as multi-passes. Another issue that needs to be considered is that for a CEAS measurement, when the absorption becomes stronger, the effective path length will also be depend on the per-pass optical loss [19]. If the total loss becomes high enough because of the absorption from a liquid sample, there will be a decrease in the total pass length as well. In this case the total 
absorbance and sample number density will no longer hold a linear relationship as the Beer's law indicates. In this work, an effective path length is always assumed to be one round trip or twice the distance between the mirror and the fibers $(1.7 \mathrm{~cm})$, so if an absorbance higher the $\mathrm{H}_{2} \mathrm{O}$ solution is measured in the fiber-loop system, an effective path length has to be calculated first.

By switching from side-polished fiber sensors to the mirror reflection device, the increase happens not only on the signal level because the light becomes more efficient, but also on the noise level as well. Clearly when the system is connected as a whole continuous piece of fiber, there will be less influence on the light intensity travelling inside the fiber than in the free space. In the mirror reflection device we will have more aspects to consider. More noise would be introduced from the sensing device because of its exposure to the surrounding environment in the lab and higher random noise from a longer time it takes to finish one sample test. Examples like the fluctuations on the fiber alignments brought by liquid flowing, dust particles sticking on the fiber ends, influence from unwanted contaminates in the liquid samples are all possible ways that could make the noise worse than a side-polished fiber sealed inside a small tubing. Flow rate on the pump is set on a low speed and sometimes samples are added manually to shorten the time it takes to finish one measurement. By switching to a newly designed Teflon container, manually adding and removing samples and carefully removing all the fiber coating inside the sensing region, we are able to minimize the noise level on the mirror reflection sensor, but it is still 10 
times larger than a side-polished fiber sensor. Further work on the mirror reflection sensor is in progress to reduce the noise.

After finishing the $\mathrm{H}_{2} \mathrm{O}$ and $\mathrm{D}_{2} \mathrm{O}$ sample pairs, we are trying to expand our work to $\mathrm{H}_{2} \mathrm{O}$ detections in organics. The organic sample we pick is carbon tetrachloride $\left(\mathrm{CCl}_{4}\right)$ for it is a widely used organic solvent but its nonpolar molecule makes it immiscible with $\mathrm{H}_{2} \mathrm{O}$. The measurement of $\mathrm{H}_{2} \mathrm{O}$ in organics will help to determine a detection limit of this device more precisely. A saturate solubility of $\mathrm{H}_{2} \mathrm{O}$ in $\mathrm{CCl}_{4}$ is at a molar fraction of $8.97 \times 10^{-4}$ at $25^{\circ} \mathrm{C}$ [20], which is $0.00924 \mathrm{~mol} / \mathrm{L}$ or switching to the volume proportion of $1.66 \times 10^{-4}$ (166ppm). According to the absorbance we have on the $1 \% \mathrm{H}_{2} \mathrm{O}$ solution, the absorbance on a saturate $\mathrm{H}_{2} \mathrm{O}$ in $\mathrm{CCl}_{4}$ solution is expected to be 0.005 to 0.01 , which is lower than the absorbance of pure $\mathrm{H}_{2} \mathrm{O}$ sample measured by a side-polished fiber sensor but still detectable. Saturated $\mathrm{H}_{2} \mathrm{O}$ solutions are made by thoroughly mixing $\mathrm{H}_{2} \mathrm{O}$ and $\mathrm{CCl}_{4}$ and pouring out extra $\mathrm{H}_{2} \mathrm{O}$ floating on top. Measurement procedures are the same as before, and the results are shown in Figure 4.4. The position of the absorption peak matches with other works done on $\mathrm{H}_{2} \mathrm{O}$ in $\mathrm{CCl}_{4}$ [18] but the absorbance appears in the figure with clear noise on it. The smoothing method of Savitzky-Golay filter [21] is applied on the curve in order to make the line shape smoother so we can have a more stable and accurate absorbance.

To confirm the absorbance of saturated $\mathrm{H}_{2} \mathrm{O}$ in $\mathrm{CCl}_{4}$ measured with this device, we further 
compare the spectrum taken in ref[18] of $\mathrm{H}_{2} \mathrm{O}$ spectra in $\mathrm{CCl}_{4}$ with the measurements got on this device. Figure 4.5 shows the spectrum of $\mathrm{H}_{2} \mathrm{O}$ solution from ref[18], and the $\mathrm{H}_{2} \mathrm{O}$ was made unsaturated at $0.0055 \mathrm{~mol} / \mathrm{L}$ (saturate solubility $0.00924 \mathrm{~mol} / \mathrm{L}$ ), and the path length was $10 \mathrm{~cm}(3.2 \mathrm{~cm}$ in our work). Our test shows a match with the absorbance at $5285 \mathrm{~cm}^{-1}(\sim 1.9 \mu \mathrm{m})$ but not the $7167 \mathrm{~cm}^{-1}(\sim 1.4 \mu \mathrm{m})$ absorbance. However the absorption coefficient for $\mathrm{H}_{2} \mathrm{O}$ at these two positions are close to each other [22] (In ref[22] the gas phase absorption coefficient for the two peaks are 21.9 and $16.1 \mathrm{~cm}^{-2} \mathrm{~atm}^{-1}$ respectively), so we believe one of the absorbance measured in ref[18] may be incorrect, and further measurements have to be done to confirm our suspicion.

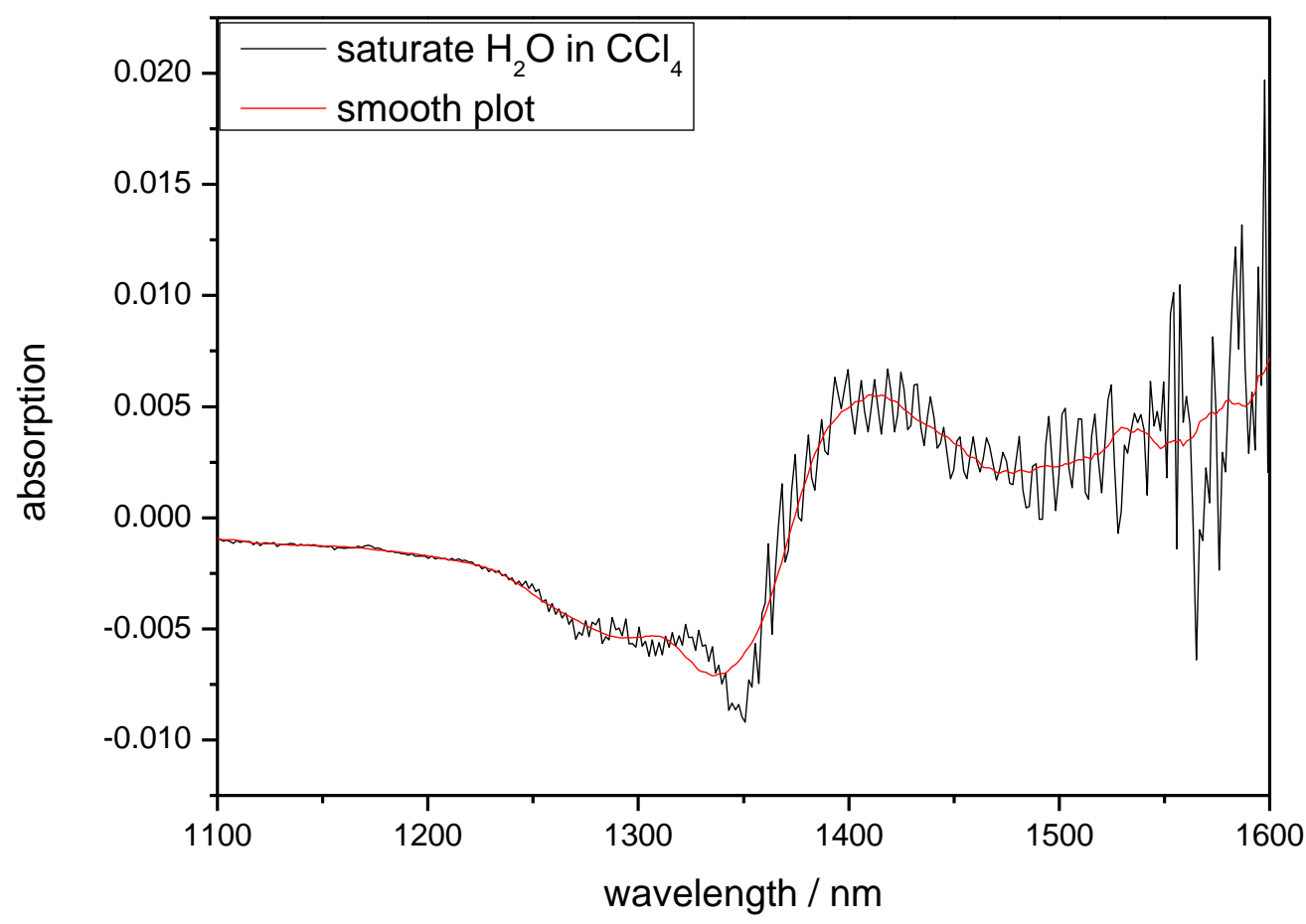

Figure 4.4: Absorbance of saturated $\mathrm{H}_{2} \mathrm{O}$ in $\mathrm{CCl}_{4}$, reference is pure $\mathrm{CCl}_{4}$. Red line is the smoothing plot. 


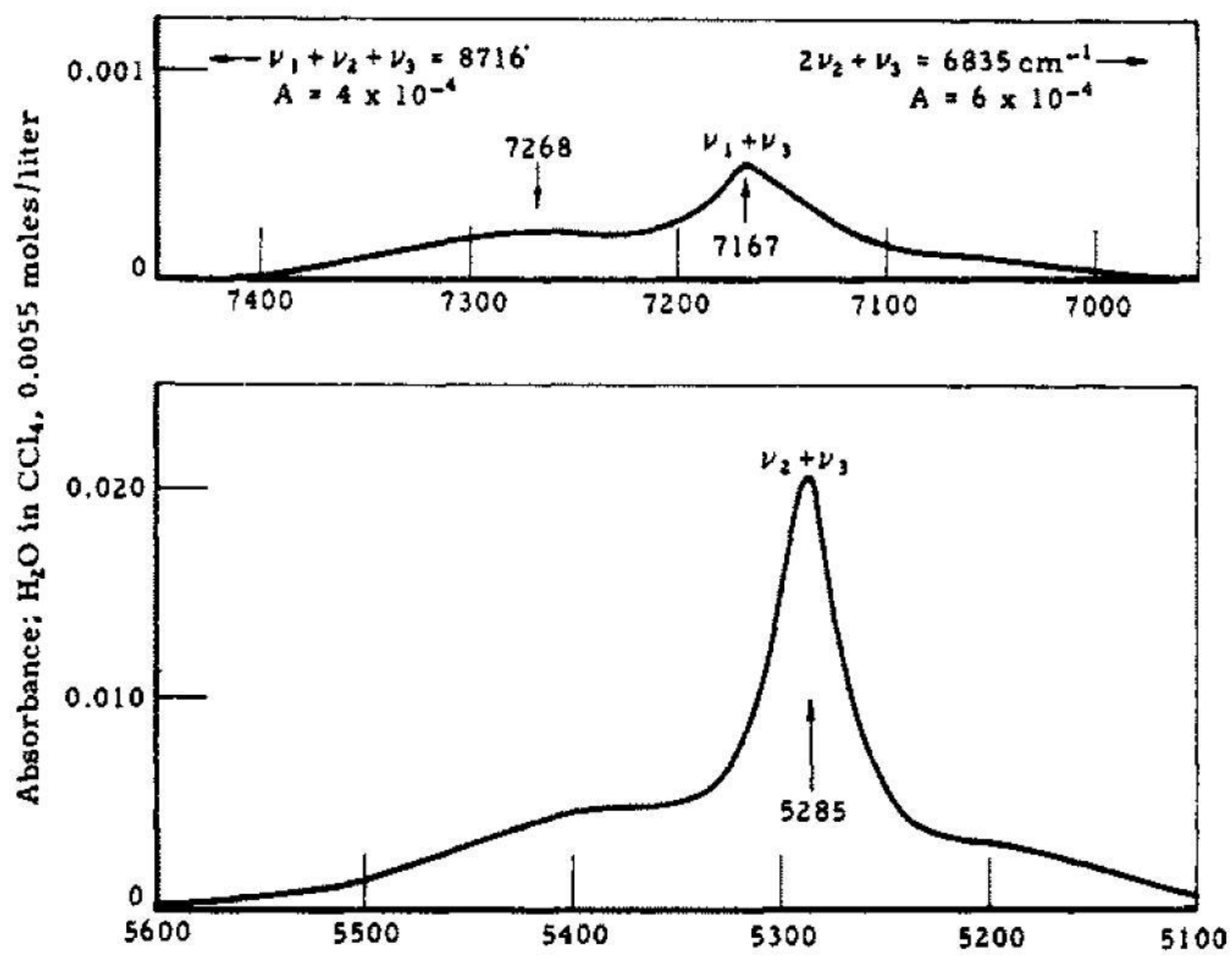

Figure 4.5: The $\mathrm{H}_{2} \mathrm{O}$ absorbance in $\mathrm{CCl}_{4}$ solutions done in ref[18]. $\mathrm{H}_{2} \mathrm{O}$ concentration is made $0.0055 \mathrm{~mol} / \mathrm{L}$ ( $59.5 \%$ of the saturate solubility), and a total path length is $10 \mathrm{~cm}$.

\subsection{Comparison of Different Sensors}

The comparison of the sensors focuses on the signal to noise level and detection limit.

Because the fiber tapers do not give a stable signal to noise ratio due to their unstable waist size, and that the issue of nonadiabatic transitions makes the final absorbance less accurate to demonstrate an actual $\mathrm{H}_{2} \mathrm{O}$ concentration, the comparison will be mostly focused on the side-polished fiber sensors and the mirror reflection device. For the mirror reflection sensor the calculation is separated to two parts of $\mathrm{H}_{2} \mathrm{O}$ samples in $\mathrm{D}_{2} \mathrm{O}$ and $\mathrm{CCl}_{4}$. Table 4.2 gives 
the signal to noise ratio and detection limit on both sensors.

Table 4.2 Signal to noise and detection limit comparison of the sensors

\begin{tabular}{|l|c|c|c|}
\hline \multirow{2}{*}{} & Pure $\mathrm{H}_{2} \mathrm{O}$ on side- & \multicolumn{2}{|c|}{ Mirror reflection sensor } \\
\cline { 3 - 4 } & polished fibers & $1 \% \mathrm{H}_{2} \mathrm{O}$ & Saturate $\mathrm{H}_{2}{\mathrm{O} \mathrm{in} \mathrm{CCl}_{4}}$ \\
\hline Signal to Noise & 100 & 125 & 2 \\
\hline Detection limit & 0.03 & 0.0002 & 0.0002 \\
\hline
\end{tabular}

From Table 4.2 it is easy to find the detection limit improvement on the device when the sensor is switched from the side-polished fibers to the mirror reflection device. Furthermore, with the efforts done on changing device materials to organic inert substances like Teflon and removing fiber coating, we are able to make a noise level for the organic sample similar as the inorganic samples like a $\mathrm{H}_{2} \mathrm{O}$ and $\mathrm{D}_{2} \mathrm{O}$ environment, but there is still a more than 10 times noise level increase when the sensor is changed. (The signal level increases $\sim 15$ times after changing to a mirror reflection sensor while signal to noise level only increases slightly.) Our work now is focusing on improving the noise level on the mirror reflection device to get it closer to the side-polished fiber sensors which have a noise level almost the same as the background noise. If the signal to noise can be further improved by 10 times higher, the detection limit can reach a level of $\sim 20 \mathrm{ppm}$, so more $\mathrm{H}_{2} \mathrm{O}$ solutions in organics can be measured by this device.

\subsection{Conclusions}

In this chapter results from the three different sensors built on the fiber-loop are 
demonstrated to indicate their working abilities and detection limits. For the fiber tapers, unexplainable peaks appear in the spectra, and the absorbance appears to be different for different tapers because some taper parameters are not well controlled. We believe the selfmade tapers failed to show the advantages of a taper sensing system by giving a relatively lower signal and higher noise than expected. The taper was replaced by a side-polished fiber from which better signal results are provided. Spectrum from pure $\mathrm{H}_{2} \mathrm{O}$ sample gives a clear absorption feature at the wavelength of $1450 \mathrm{~nm}$ and a signal to noise of 100 is reached with the pure sample. Tests of mixed samples of $\mathrm{H}_{2} \mathrm{O}$ and $\mathrm{D}_{2} \mathrm{O}$ indicate a slight increase in the noise level but a limit of concentration at $<5 \%$ of $\mathrm{H}_{2} \mathrm{O}$ is reached so far on this sensing element. The mirror reflection device is installed because a side-polished fiber can make use of only $0.02 \%$ of the supercontinuum light source in the evanescent field. By changing to free space coupling, improvement on the signal level is observed. Measurement on $1 \% \mathrm{H}_{2} \mathrm{O}$ sample in $\mathrm{D}_{2} \mathrm{O}$ gives an increase of $\sim 15$ times in absorbance compared with a pure sample measured by a side-polished fiber. Further tests with organics shows it has the ability for $\mathrm{H}_{2} \mathrm{O}$ detection in organics immiscible with $\mathrm{H}_{2} \mathrm{O}$. An issue of relatively high noise level is observed and some efforts are done to minimize it. In sum, each sensor has its own advantages and limitations, and we believe there is still room of improvements for all three methods. 


\subsection{Reference}

1. Mumma, Michael J., Harold A. Weaver, Harold P. Larson, D. Scott Davis, and Michael Williams. "Detection of water vapor in Halley's comet." Science (1986): 1523-1528.

2. Pope, Robin M., and Edward S. Fry. "Absorption spectrum (380-700 nm) of pure water. II. Integrating cavity measurements." Applied optics 36, no. 33 (1997): 8710-8723.

3. Hagen, W., A. G. G. M. Tielens, and J. M. Greenberg. "The infrared spectra of amorphous solid water and ice Ic between 10 and 140 K." Chemical Physics 56, no. 3 (1981): 367-379.

4. Falk, Michael, and T. A. Ford. "Infrared spectrum and structure of liquid water." Canadian Journal of Chemistry 44, no. 14 (1966): 1699-1707.

5. Curcio, Joseph A., and Charles C. Petty. "The near infrared absorption spectrum of liquid water." JOSA 41, no. 5 (1951): 302-304.

6. Pfeiffer, Heinz G., and Herman A. Liebhafsky. "The origins of Beer's law." J. Chem. Educ 28, no. 3 (1951): 123.

7. Ahmad, Mohammad, and Larry L. Hench. "Effect of taper geometries and launch angle on evanescent wave penetration depth in optical fibers." Biosensors and Bioelectronics 20, no. 7 (2005): 1312-1319.

8. Mirabella Jr, F. M. "Internal reflection spectroscopy." Applied Spectroscopy Reviews 21, no. 1-2 (1985): 45-178.

9. Tian, Zhaobing, Scott S-H. Yam, Jack Barnes, Wojtek Bock, Patricia Greig, James M. Fraser, Hans-Peter Loock, and Richard D. Oleschuk. "Refractive index sensing with 
Mach-Zehnder interferometer based on concatenating two single-mode fiber tapers." IEEE Photonics Technology Letters 20, no. 8 (2008): 626-628.

10. Bobb, Lloyd C., P. M. Shankar, and Howard D. Krumboltz. "Bending effects in biconically tapered single-mode fibers." Journal of lightwave technology 8, no. 7 (1990): 1084-1090.

11. Tseng, Shiao-Min, and Chin-Lin Chen. "Side-polished fibers." Applied optics 31, no. 18 (1992): 3438-3447.

12. Yamatera, Hideo, Brian Fitzpatrick, and Gilbert Gordon. "Near infrared spectra of water and aqueous solutions." Journal of Molecular Spectroscopy 14, no. 1-4 (1964): 268-278.

13. Hulston, J. R. "Revised Zero-Point Energy Calculation for $\mathrm{H} 2 \mathrm{O}+\mathrm{D} 2 \mathrm{O} \leftrightharpoons 2 \mathrm{HDO}$ Isotopic Equilibrium." The Journal of Chemical Physics 50, no. 3 (1969): 1483-1484.

14. Chen, Bin, Ivaylo Ivanov, Michael L. Klein, and Michele Parrinello. "Hydrogen bonding in water." Physical Review Letters 91, no. 21 (2003): 215503.

15. Kieu, Khanh Quoc, and Masud Mansuripur. "Biconical fiber taper sensors." IEEE Photonics Technology Letters 18, no. 21 (2006): 2239-2241.

16. Gravina, Roberto, Genni Testa, and Romeo Bernini. "Perfluorinated plastic optical fiber tapers for evanescent wave sensing." Sensors 9, no. 12 (2009): 10423-10433.

17. Tarsa, Peter B., Paul Rabinowitz, and Kevin K. Lehmann. "Evanescent field absorption in a passive optical fiber resonator using continuous-wave cavity ring-down spectroscopy." Chemical Physics Letters 383, no. 3 (2004): 297-303. 
18. Stevenson, D. P. "On the monomer concentration in liquid water." The Journal of Physical Chemistry 69, no. 7 (1965): 2145-2152.

19. Seetohul, L. Nitin, Zulfiqur Ali, and Meez Islam. "Liquid-phase broadband cavity enhanced absorption spectroscopy (BBCEAS) studies in a $20 \mathrm{~cm}$ cell." Analyst 134, no. 9 (2009): 1887-1895.

20. "IUPAC-NIST Solubility Database NIST Standard Reference Database 106." Available at: https://srdata.nist.gov/solubility/sol_detail.aspx?sysID=60_23

21. Luo, Jianwen, Kui Ying, and Jing Bai. "Savitzky-Golay smoothing and differentiation filter for even number data." Signal Processing85, no. 7 (2005): 1429-1434.

22. Ferriso, C. C., and C. B. Ludwig. "Spectral Emissivities and Integrated Intensities of the 1.87-, 1.38-, and 1.14- $\mu \mathrm{H} 2 \mathrm{O}$ Bands between $1000^{\circ}$ and $2200^{\circ} \mathrm{K} . "$ The Journal of Chemical Physics41, no. 6 (1964): 1668-1674. 


\section{Chapter 5 Applications and Future Work}

After finishing the liability tests of the setup the next steps is to figure out in which areas this liquid phase fiber-loop cavity enhanced absorption spectroscopy device can be used and how well the working ability is and in what aspects there is still improvements needed. An advantage of this device is that it is easy to be made compact to work properly without any further alignment, especially for the side-polished fiber sensor. Every fiber component can be connected together by a fusion splicer so finally the whole device can be all made from optical fibers. This makes it possible to make it portable and to be used in situations not only in a research lab. There are also several issues need to be solved as well. Some future work will still be done focusing on these problems.

\subsection{Future work}

There are still several important issues left to be solved before this project can be finished.

Recently most of the work is focused on lowering noise levels, making smaller device sizes and designing a more efficient taper pulling device. The noise levels are much lower now than the time when the system was first set, but it is still higher than expected, especially for the mirror reflection sensor. For a side-polished fiber sensor, the noise is fair to be slightly higher than the noise from a blank cavity. Because only inorganic samples of $\mathrm{H}_{2} \mathrm{O}$ and $\mathrm{D}_{2} \mathrm{O}$ are tested on this setup, we cannot make any comparisons on the organics done on the mirror reflection device. But we believe some adjustments have to be done if the 
side-polished fiber sensor is to be expanded to organic samples. To keep a stable spectrum, the whole flow system have to be changed to materials with higher quality of chemical resistance. Most kinds of conventional tubing are not suitable for organics solvents like carbon tetrachloride $\left(\mathrm{CCl}_{4}\right)$ used in this project. Fiber coating also has to be removed for the whole region inside the flow system. The core and cladding of fibers are resistant to most chemicals because of their material (silica), but the coating is usually made from plastic polymers like acrylate or polyvinyl chloride (PVC), which can be severely damaged when immersed in some organics. We think this is the reason why the noise level on organic samples spectra from the mirror reflection device is high when it is first built. When the container is rebuilt with Teflon and the tubing is removed, noise level decreases dramatically on the mirror reflection device.

Another reason for a higher noise level is the open system for the mirror reflection sensor. A system exposed outside can have several issues against a closed system like the flow system built for a side-polished fiber sensor. A fiber sensor in a closed system can be mostly kept away from the outside environment, so we can make sure the whole device is inert to unexpected changes like temperature, humidity or random wind caused by people walking by. For an open system, however, there are more variables that can make influence on a final measurement. The $\mathrm{H}_{2} \mathrm{O}$ solutions for example, could be affect by water vapor in the air, especially in a very low concentration level. For now we are still in need of an open system for the alignment of the holder with two fibers, but the idea is to design a tubing- 
shaped container for us to insert the two fibers and mirror on both ends, so a closed device can be built when lower sample volume is consumed at the same time. A fully closed setup also helps to build a fiber connected device which can be finally portable and does not need further alignments when fully installed.

The last thing we are working on now is to rebuild the taper pulling device. Generally speaking, despite that the fact that a side-polished fiber is easier to handle than a fiber taper, the fiber taper is considered to be a better sensor for evanescent wave spectroscopy works. Compared with only $0.02 \%$ evanescent field we get on a side-polished fiber in this project, the intensity could be orders of magnitude higher on a fiber taper. We are now working on a design of a more precise control on the pulling process. Not only the pulling loss, but also the pulling distance and waist diameter should be monitored. There are ways that a flame size and temperature can be controlled [1], which will help control the total length of a taper and its final waist. A camera with microscope is needed to monitor the pulling region in the process, so both fiber core and cladding can be watched to decide if it is still adiabatic. Tapers no longer adiabatic will be recognized as failures so to improve the experimental efficiency. The two fiber clamps have to be aligned in the same direction as the half-left half-right screw. A very small angle will cause a bending on a taper in its pulling and the bending will usually lead to a nonadiabatic fiber. The more straight a fiber is held, the more possible a taper can be pulled successfully. 


\subsection{Potential Applications}

Because our project is mostly focused and done on $\mathrm{H}_{2} \mathrm{O}$ so the first application we think of is on $\mathrm{H}_{2} \mathrm{O}$ detection. The absorption feature of $\mathrm{H}_{2} \mathrm{O}$ at $1450 \mathrm{~nm}$ is relatively strong so it helps to enhance the lowest concentration this device can reach. The peak wavelength is something need to be paid attention to since it is moving with different solvents and concentrations. Because other O-H bonds also have similar absorbing features in the almost the same region of 1300 to $1450 \mathrm{~nm}$, which will cause confusion, it rules out some potential sample pairs such as the most common ones of water mixed with methanol, ethanol and other alcohols.

A great example this work can be fit in is $\mathrm{H}_{2} \mathrm{O}$ detection in gasoline. As we know gasoline is mostly composed by alkanes and other hydrocarbons. Even mixed with a slight amount of water will cause a shake or even explosion when burning inside engine. $\mathrm{H}_{2} \mathrm{O}$ solubility in gasoline can be as high as $0.5 \%$ in volume when $10 \%$ ethanol $[2,3]$ is contained in gasoline today. And because water tolerance [4] is much lower than this level, it is important to make accurate measurements on $\mathrm{H}_{2} \mathrm{O}$ concentrations. Another similar example is for detection in oils. Pure oil like machine oil or lubricating oil [5] could be easily contaminated when mixed with water, especially when the water is not pure. However for oils there are usually groups like carboxyl in it so we are not sure if the spectrum range is clean enough for detections. If most light is absorbed by the solvent itself, it would not make a good idea to work in this region. 
Our work is restrained by the wavelength coverage of the supercontinuum light source and the wavelength coverage of the spectrometer. There are other supercontinuum sources reported to have a coverage larger than ours or in different wavelength ranges. [6, 7] There are also manufactured fiber based supercontinuum sources $[8,9]$ that cover the near infrared region or other parts of the whole light spectrum. Other light sources like a halogen source [10] makes other options since they are usually more stable than a supercontinuum but with lower intensities and worse directionality. A spectrometer can also be changed easily without changing other components in the whole device. Thus it would not be very difficult to do same measurements at different wavelengths. If the work is not restricted by wavelength coverage and samples, the applications can be made more broadly.

\subsection{Conclusions}

There are still works that can be done to improve the results we have from this project. The redesign on the pulling device and efforts on reducing the noise are only parts of them. Our final goal is to make this whole device potable to do measurements on more liquid compounds and their mixtures to give simultaneous results. If the whole system can be exchanged to fiber-based components, it would make it possible to achieve this goal. When expanding from detection of $\mathrm{H}_{2} \mathrm{O}$ to other compounds and from $1300 \mathrm{~nm}$ to $1500 \mathrm{~nm}$ to other regions in the near infrared, we believe the use of this device can be widely expanded. Similar as other works, this device can be fit in areas like concentration and absorptivity 
detection [11], refractive index measurement [12], sensor evaluations [13] and other liquid phase spectroscopy tests. The sensitivity of this device may not be able to match with some conventional and well developed sensing methods like chromatography [14], but it has own advantages of simple setup and more importantly, less expensive to build. If the sensibility can be further improved, this simple but robust device can have more application prospects.

\subsection{Reference}

1. Harun, S. W., K. S. Lim, C. K. Tio, K. Dimyati, and H. Ahmad. "Theoretical analysis and fabrication of tapered fiber." Optik-International Journal for Light and Electron Optics 124, no. 6 (2013): 538-543.

2. Shiu, Wan Ying, Mark Bobra, Alice M. Bobra, Aila Maijanen, Leena Suntio, and Donald Mackay. "The water solubility of crude oils and petroleum products." Oil and Chemical pollution 7, no. 1 (1990): 57-84.

3. Mužíková, Zlata, Milan Pospíšil, and Gustav Šebor. "Volatility and phase stability of petrol blends with ethanol." Fuel 88, no. 8 (2009): 1351-1356.

4. Gramajo de Doz, Mónica B., Carlos M. Bonatti, and Horacio N. Sólimo. "Water tolerance and ethanol concentration in ethanol-gasoline fuels at three temperatures." Energy \& fuels 18, no. 2 (2004): 334-337.

5. Alford, Harvey E., and Liao Chien-Wei. "Lubricating oil additive and oil containing the same having improved water tolerance." U.S. Patent 2,841,551, issued July 1, 1958.

6. Woodward, John T., Allan W. Smith, Colleen A. Jenkins, Chungsan Lin, Steven W. 
Brown, and Keith R. Lykke. "Supercontinuum sources for metrology." Metrologia 46, no. 4 (2009): S277.

7. Thapa, Rajesh, Dan Rhonehouse, Dan Nguyen, Kort Wiersma, Chris Smith, Jie Zong, and Arturo Chavez-Pirson. "Mid-IR supercontinuum generation in ultra-low loss, dispersion-zero shifted tellurite glass fiber with extended coverage beyond $4.5 \mu \mathrm{m} . "$ In Proc. SPIE, vol. 8898, p. 889808. 2013.

8. Available at: http://www.nktphotonics.com/lasers-fibers/en/productcategory/supercontinuum-lasers/

9. Available at: https://www.thorlabs.com/newgrouppage9.cfm?objectgroup_id=5519

10. Buschmann, Jeffrey B., Kevin R. Davies, and James E. Oetken. "Tungsten halogen lamp." U.S. Patent D508,575, issued August 16, 2005.

11. Blaikie, Thomas PJ, John Couper, Gus Hancock, Philip L. Hurst, Robert Peverall, Graham Richmond, Grant AD Ritchie, David Taylor, and Kevin Valentine. "Portable Device for Measuring Breath Acetone Based on Sample Preconcentration and Cavity Enhanced Spectroscopy." Analytical chemistry 88, no. 22 (2016): 11016-11021.

12. Bescherer, Klaus, Jack A. Barnes, and Hans-Peter Loock. "Absorption measurements in liquid core waveguides using cavity ring-down spectroscopy." Analytical chemistry 85, no. 9 (2013): 4328-4334.

13. Fiedler, Sven E., Achim Hese, and Albert A. Ruth. "Incoherent broad-band cavityenhanced absorption spectroscopy of liquids." Review of scientific instruments 76, no. 2 (2005): 023107. 
14. Swartz, Michael E. "UPLC ${ }^{\mathrm{TM}}$ : an introduction and review." Journal of Liquid Chromatography \& Related Technologies 28, no. 7-8 (2005): 1253-1263. 


\section{Acknowledgements}

This project can be done only with the help of many people. I would like to thank my advisor, Dr. Kevin Lehmann, for his guidance throughout the whole process of this project.

I would also like to thank all the current and previous group members of the Lehmann group for their help and support in finishing this work. Thanks you to Dr. Brooks Pate, Dr. Ian Harrison, Dr. Andreas Gahlmann and Dr. Bob Jones for both serving in my research committee and support in my work. Thanks to the professors and graduate students from the Chemistry and Physics Department of the University of Virginia for the help when I come to them with questions.

This project cannot be done without the help of people from Tiger Optics. Thank you to Dr. Helen Waechter, Dr. Florian Alder and Dr. Ryan Matz for the guidance and help when I come up with problems in this project. With the knowledgeable advice I am able to overcome the obstacles one after another in finishing this project. This work is made possible because of your help.

I would like to thank my family as well. Thank you to my wife, Erxin Zhou, for your pushing and encouraging for the past four years, and for giving birth to our new baby boy Charles this month. Thank you to my parents, for supporting me in pursuing my academic development far away from them. My work and life will be a whole lot different without the support from all of you. 